$+\infty$

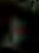

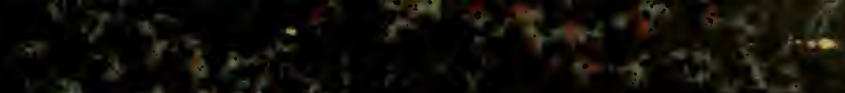

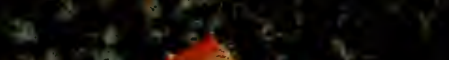

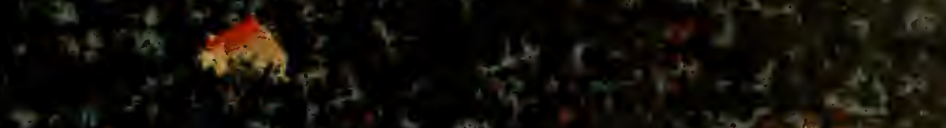

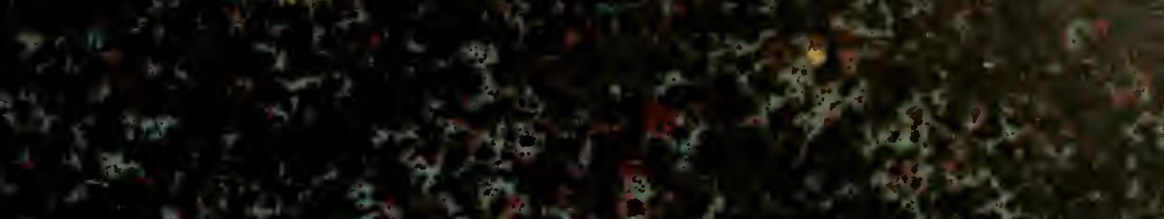

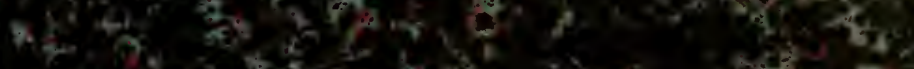

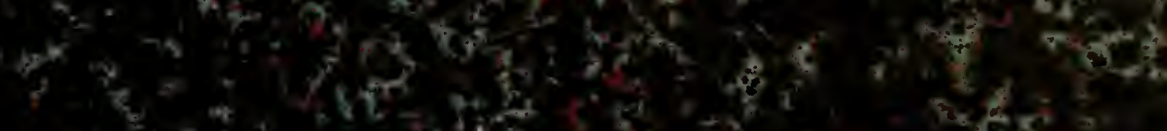

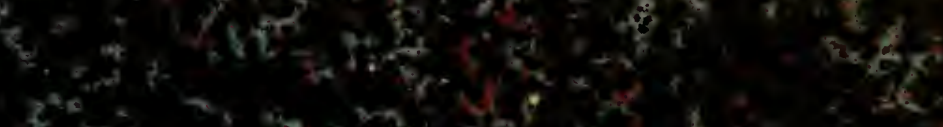

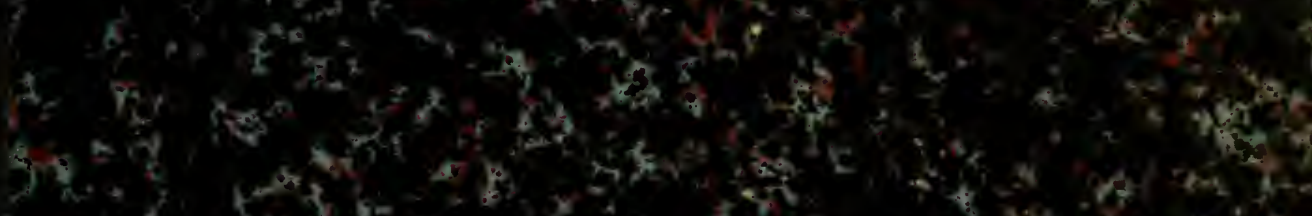

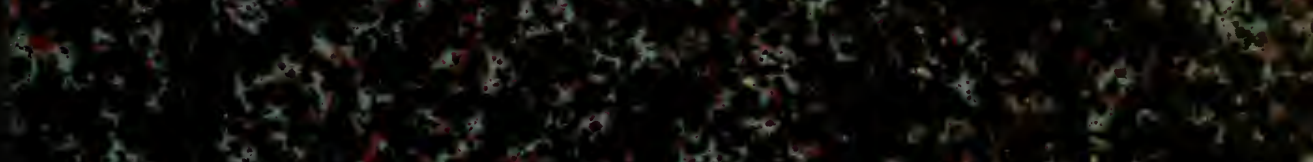

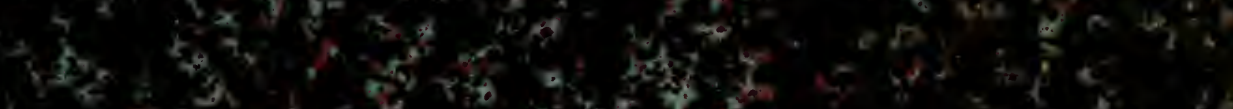

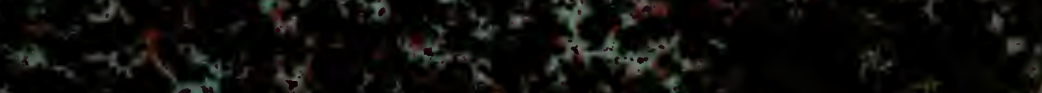

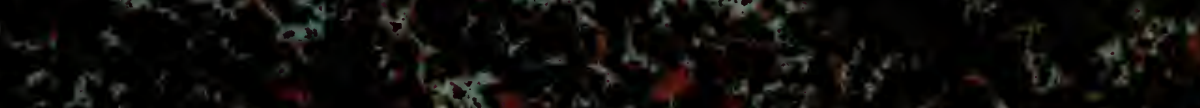

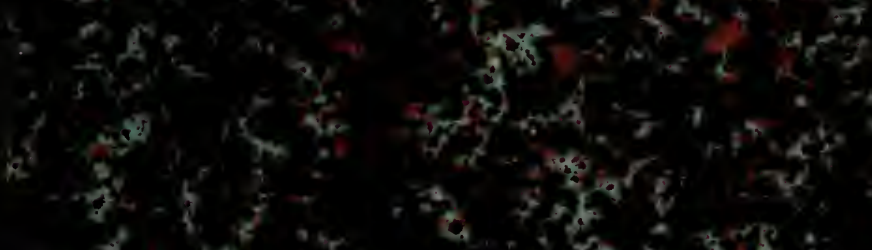

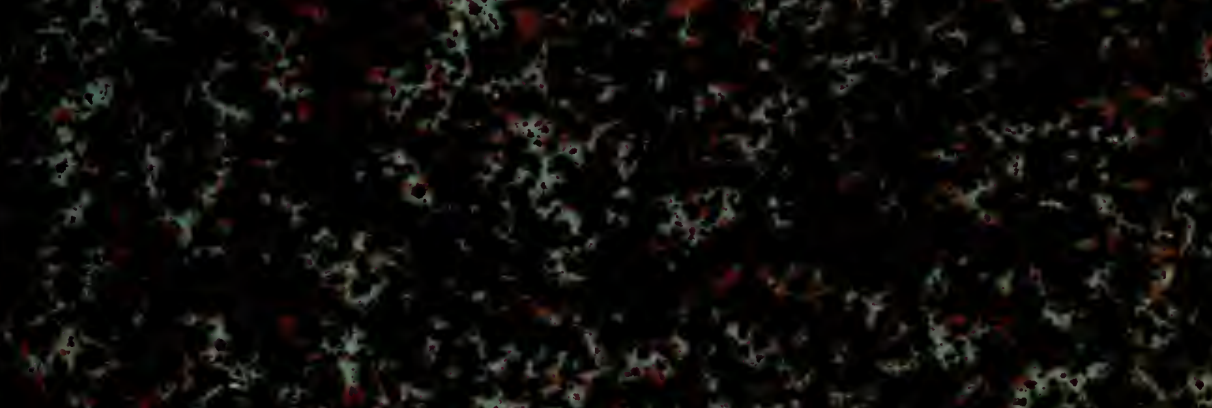

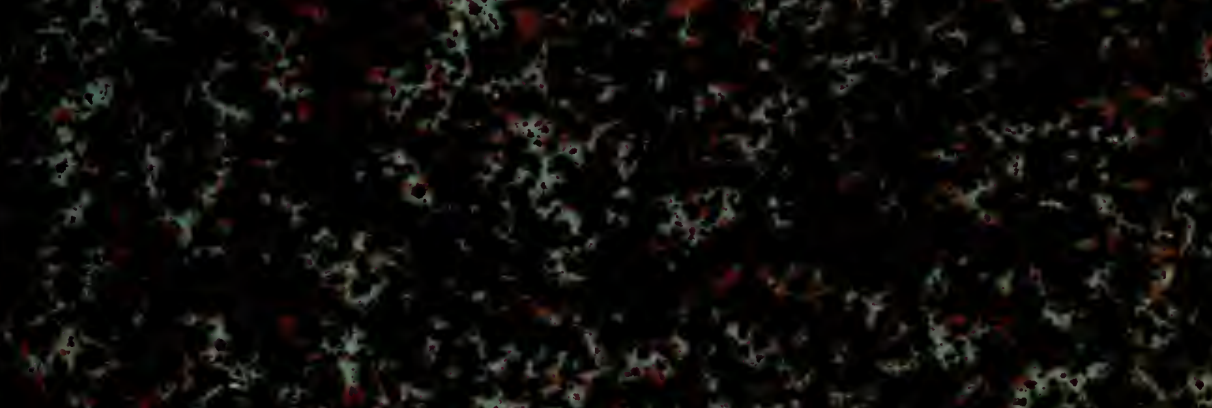

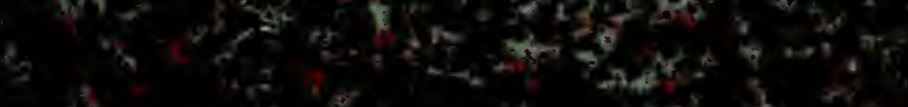

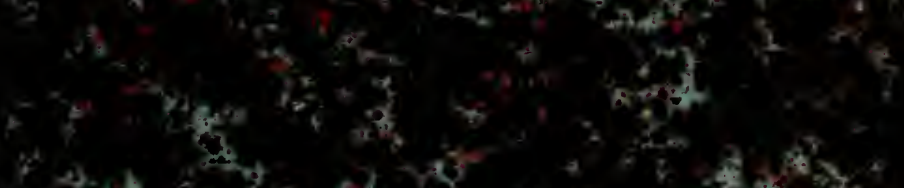

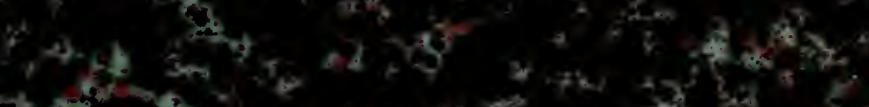
की

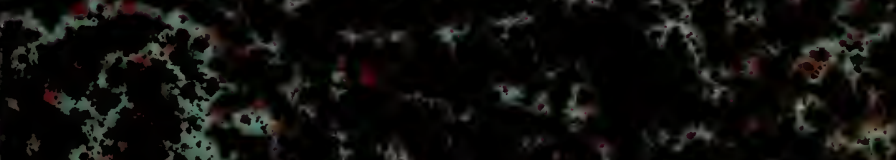

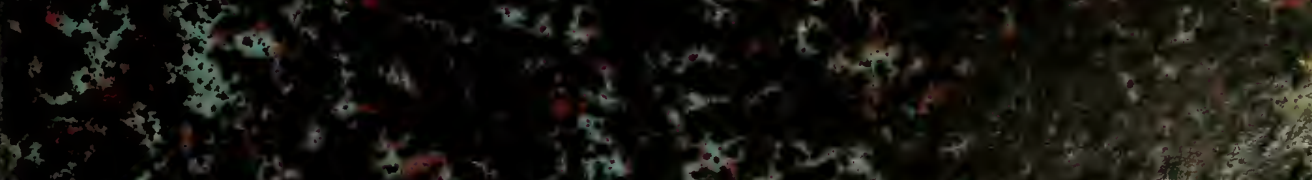

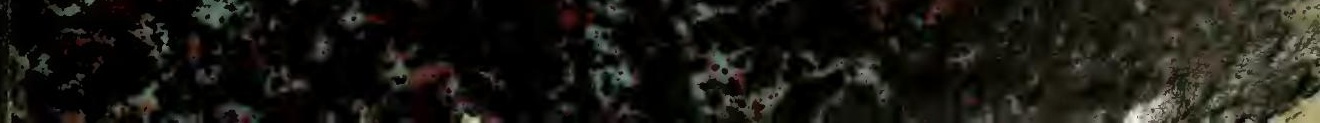




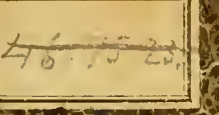

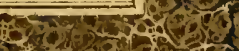

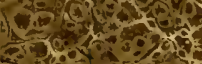

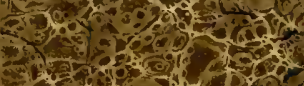

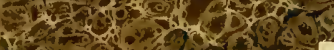

Special Collectrons

$\tan 6 x^{2}-25$

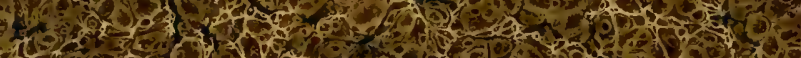

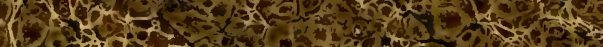

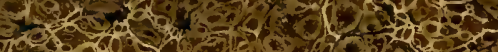

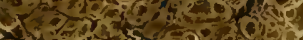

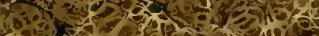

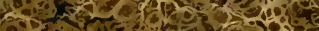

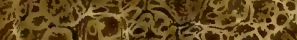

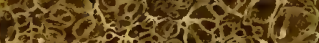

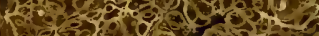

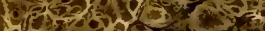

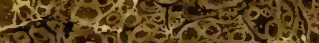

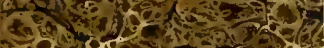

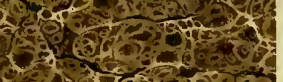

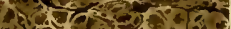
$40,15 y)-205023$

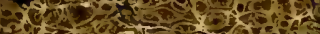

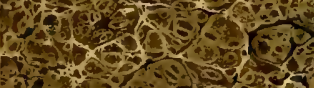
4. 3 - 5 की

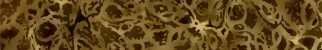

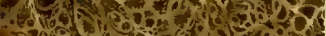

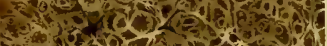

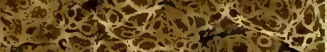
G whos

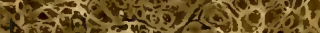
Jo, -1.03 oferorat

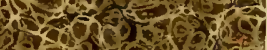
W.

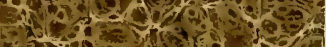

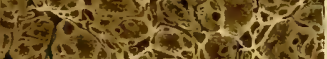

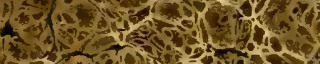

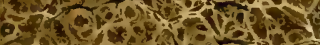

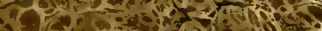
3.

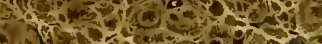

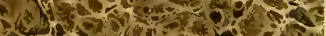

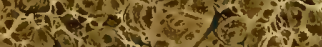

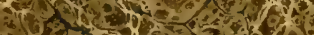

$$
k+2920
$$

$4 C Z$

LIERARY

WNAVAE
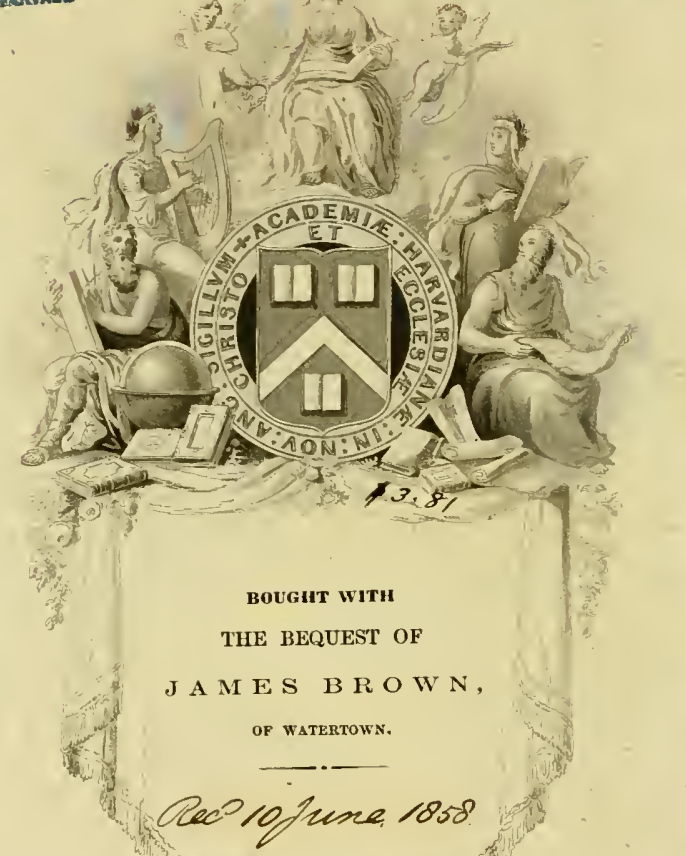

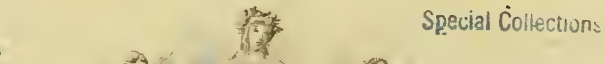

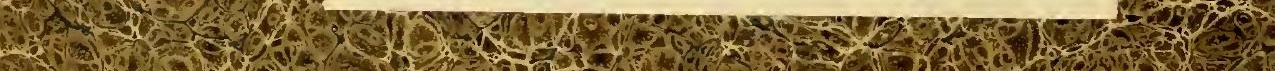

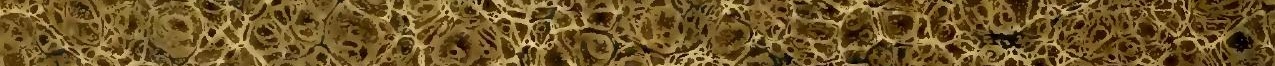

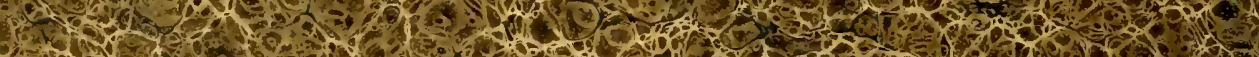

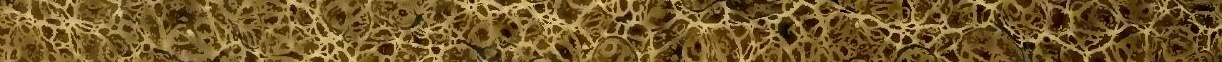

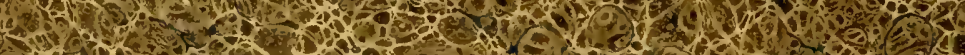

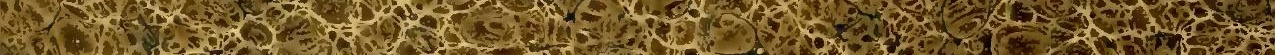
Fen

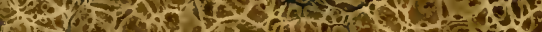

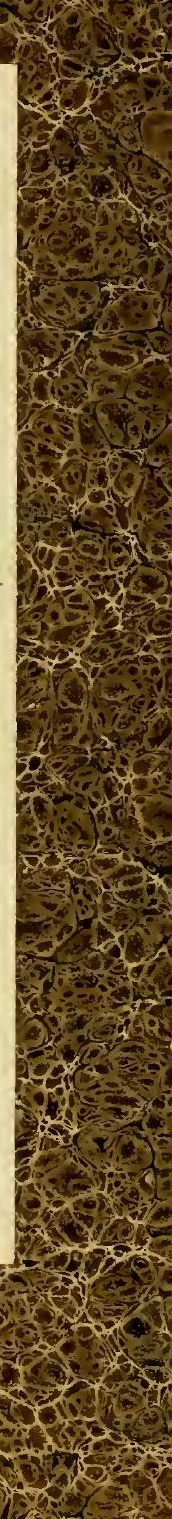




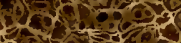 \\ 5. \\ 5 \\ (3)}

(2)

H.t.

2.

a

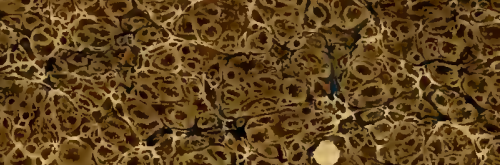
for H.

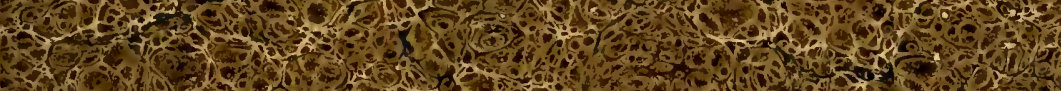

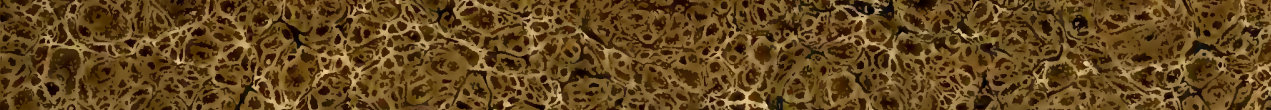
1. -1.7. 3.

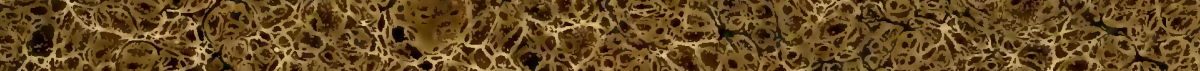
16.7.

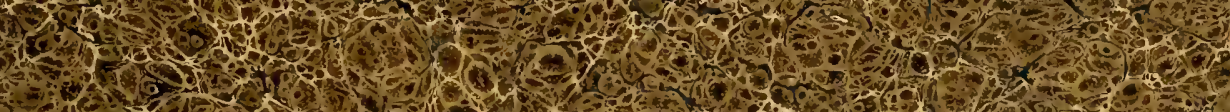
2. con. ore 2. 1. 





\section{T E S T A C E A M I C R O S C O P I C A}

A L I A Q U E M I N U T A - EX GENERIB U S

ARGONAUTA ET NAUTILUS AD NATURAM DELINEATA ET DESCRIPTA
A
L EOPOLDOAF I C H T E L
E T
JO. PAULO CAROLO A MOLL.
CUM 24 TABULIS AERI INCISIS.

- M i c r o $\int \mathrm{cop}$ i $\int \mathrm{ch}$ e und andere kleine

$\mathrm{S}$ c h a $1 \mathrm{th}$ i e $\mathrm{r}$ e aus den Gefchlechtern

Argonaute und Schiffer,

nach der Natur gezeichnet und befchrieben

$$
\text { Leopold v. Fichtel, }
$$

Mitglied der Linneifchen Gefellychaft zu London, und der Afiatifchen zu Calcutta u n d

Joh. Paul Carl v. Moll. Mit $\backslash 24$ Kupfertafeln.

$\mathrm{W}$ i e $\mathrm{n}, \mathrm{I} 803$.

In der Camefinaifchen Buchhandlung. 


\section{PRAEFATIO.}

Interea, cum hifforize naturalis amor in $B_{y} y$ den fo allgemein ausgebreiteten tot hominum animis difpanfus fit ac in Hong aur Naturgefohichte, dar fich fuft illius fudio fere omnes occupentur, vel jedermann mit ihrer Erlernung besthiftieam faltcm pro oblcctamcnto habeant, get and fie aun Gegenflande des Verginiifane admodum miraremur, in defcribendis geiss mochet, follte man fich billig äth\& repræfentandis nature rebus magnam fierft windern, daßs in Befihreibung und

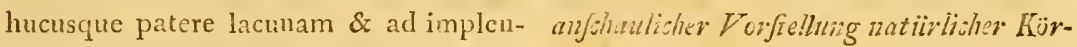
dum hoc vacuum vix factum efie ini- per 200 sh immer eine farke Liicke offen tium, licet pars ifta, quam ftatim no- geblieben und diefer leeve Theil hann minabimus, tot pulchra oculis animo- angefangen worden it ausgefiillet zul que præbeat objecta $\&$ ad creatoris ipfo- werden, da er doch fo viel Schönes fiir rum glorificationem permultum conferat. das Auge und Genuith darreicht und aur Loquimur de minutis iftis fape armato Verherrlichung feines grofsen Urhebers folum oculo vifibilibus aut cognofcibili- fo viel beyträgt. Wir meynen die hleinenz bus animalibus teftaccis, vel faltem eo- oft wur dem bewafineten Auge fichtbaren rum fuffulcris quafi offeis feu fceietis, oder kenntlichen Schalthiere, oder wenigqure vulgo domunculorum five tefiarum fens ihre Beingeriifte, die man insgemein nomine veniunt. An horum exifentia Gehiüufe oder Schulen nennt. Sollten die$x^{2}$. 
absque ullo effet fcopo ufuque determi- fe wohl ohne Eataweck da fegn, oder ihnato, rel ob parram corporis fui mo. res kleinzen Boues wegen weniger Betrachlem \& ftructuram corpufcula hæc mino- tung zerdienen, als die von größeren Unirem mererentur attentionem, quam ea, fange? In den klcinften Dingen aeigt die quæ majore gaudent amplitudine? In Natur oft ihre roræïglichfte Größe. Und minimis natura faepe maxima. Ecquid was ift in der Natur grofs? Was klein? in natura magnum? quidve parvum? Warum follen diefe artigen Gefü̈opfe, die Quam ob rem creatura he formofifi- wiv in Beaug auf uns oder andere Wefen mæ, quas in relatione ad nos velad alia klein nennen, die aber an Schünheit ihres entia parvas rocamus, quæ vero fru- Baues oder Verfchiedenheit des Farbencturae elegantia vel colorum varietate ma- Spiels den gröjsern nichts nachgeben, auch joribus neutiquam cedunt, imo fæpius fie wohl öfters atiertreffen, das unwürantecellunt, immeritam experiantur for- dige Schichal halen, nicht befolrieben oder tem, ut non defcribantur, vel iconibus repræententur, cum tamen honor hicce rel potius hoc jus aliis minutis creaturis, polypis nimirum, aliisque zoophytis, ut $\&$ infectis permultis vicibus ceferit? Diximus, quod mirari deberemus, hanc hiftoria naturalis partem adeo effe neglectam; fed multum ponderis fui amittet admiratio, fi perpendas, nultos perquifitioni huic fefe fubtrahere partim timore affectos, ne crebro microfcopii ufu oculis noceant, partim alios commoditati nimis indulgentes, cum magis iis ar- durch den vielen Gelrauch des Vergröfrideat, fi absque ulla mora \& labore ob- ferungsglafes $\approx u$ fchaden, theils aus Bejecta obria oculis admodum palpabilia quemlichkeit, weil es leithter ift, gleich polfunt contemplari, aut ctiam alios, ohne Unftände eincn ftark in dic Augen quia oeconomicam rel phyfacam exinde fallenden Gegenfand letrachten $\approx$ h bör- 


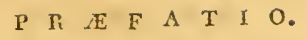

inmediate redundantem non comprehendere polfunt utilitatem.

Non omnino quidem aflerendi nobis animus eft, plane neminem hucusqiie fuife, qui ejusmodi operi admoviffet manum. Fuit Fanus Plansus 1), cft Abbas Soldani 2) \& adluuc noviores Boys \& Walker 3). Sed preterea quod prior aliquot paucas folummodo fpecies publicaverit iconesquc mediocres \& partim malas dederit, alter autem ad certas folum regiones fe reftrinxerit ficque omni cum fua diligentia magnum adhuc fpecierum numerum reliquerit, neque icones femper fatis accurate fuccellerint \& colores prorfus deficiant, etiam ambo fcriptores non methodo \& genio nunc domi-

nen; oder auch einige, weil fie den ökononifishen oder phyfifshen Nutzen nicht gleich unnittelbar daron einfehen.

Wir wollen swar nicht gans behaupten, days fich noch gar. Nienand gefunden habe, der fich an diefes Fach gewaget hiitte. Wir haben einen Janus Plancus 1), einen Soldani 2), wnd noch neuer einen Boys und Walker 3). Allein aufserdem, dafs der erftere nur etiche wenige Arten angcfilhret und fehr fohlecht abgebildet, der zueyte auch fich nur auf gcroifse Gegenden eingefonräket und alfo bey allen feinem Fleifse noch viele Arten iibrig gelafjen hat, auch die Abbildungen nicht alle allsu genau gerathen find und die Far-ben gänslich fehlen, haben auch beyde wioht in dem jetat herrf henden Geife und

I) Fani Planci (Giovanni Bianchi) Ariminenfis de Conchis minus notis liber \&c. Edit. 2. Roma 1761. 4. cum tab. æn.

2) Saggio orittografico ovvero Offervazioni fopra le terre nautilitiche ed ammonitiche della Tofcana \&c: dal P. D. Ambrogio Soldani Ab. Camaldol. in Siena 17\&o, 4to, cum tab. æn. (Teft. folum folli.). Ejıslem Teftaceographiæ \& Zoophytographize parva \& microfcopicæ \&c. Tomi I. Pars I. Senis 1789. Pars IL. ibid, 1791. fol. cum nult. tab. æen.

3) Teftacea minuta rariora nuperrime detecta in arena littoris Sandvicenfis a Gul. Boys Arm. S. A. S. Multa addidit \& omnium figuras ope microfcopii ampliatas accurate delineavit Ge. Walker; $\&$ angl. A Collection of the Minute and Rare Shells, lately discovered in the fand of the Sea Shore near Sandwich by Wriliam Boy's, Efq. F, S. A. confiderably angmented and all their figures accurately drawn, as magnified with the Microfcope, by Geo. Walker, Bookfeller a Faversham. London 4to. cum tab. æ⿰. Opufculum hocce nodo nuperrime, abfoluto jam noftro, ex Anglia ad nos pervenit. 
nanti fatis convenienter, nec ita determi- mit der erforderlichen Genauigkeit, benate, quam nunc requiritur, præfertim fonders erfterer, ihre Gegenftände geordprior, objecta fua ordinarunt. Tertius net und befchrieben. Auch der dritte le. quoque exiguam folum tractavit regio- handelt nur eine lileine Gegend, befinreilt nem, nimis breviter defcripfit, objectà, di. Gegenftünde $\approx u$ kur $\approx$ und liefert fie ohne eorumque icones fine coloribus \& partim non fatis fideliter repræentavit, uti præ ceteris ex. gr. ex fig. $63-67$ apparet, quarum $\sigma_{3} \& \sigma_{4}$ Nautilum Beccarii Lim. (nofirum Hammonium Beccarium), fig. 65. N. crifpum L. \& fig. 66,67 Naut, Calcar L. denotant.

Porro Otto Frid. Miillerus varia tefacea minuta defcripfit, fed paucas icones dedit 4); Schrceterus etiam multa protulit, partim colorata 5); Spengle- fezzsilluminirte, geliefert 5); Spengler, vus, a quo multa aliquando exfpectari ron' dem man mit der Zit wiel erwarten poffent, adhucdum quoque folum non- künnte, hat uns ebenfalls bisher nur nit nullis, licet rariflimis, nos donavit 6); etlichen, aber fehr feitenen, befichenhet 6);

4) Von Würmern des füfen und falzigen Waffers, mit Kupfern, yon Oito Fridr. Miller. Kopenhagen 177 I. $4^{\text {to. }}$

Ejusd. Vermium terreftrium \& fluviatilium 〔eu animalium infuforiorum, helmin. thicorum \& teftaceorum, non marinorum, fuccincta hiforia. Vol, I. Pars I. Havniæ \& Lipfiæ 1773. Pars II. ibid. 1774. Vol. II. ibid. eod. anno 4'o.

Ejusd. Zoologiæ danicæ Prodromus f. animalium Daniæ \& Norvegiæ indigenarum characteres, nomina \& Cynonyma inprimis popularium. Haviae 1766.8 .

5) Die Gefcbichte der Flufsconclyylien init vorziglicher. Rüickficht anf diejenigen, melche in den thibvingifchen Waffern leben, von $\mathcal{Z}_{0}$ b. Sam. Schröter. Halle 1779. 4. wit II Kunfeitafeht.

Ejusd, minores tractatus hinc inde in majoribus operibus fuis.

6) Spengler (Lorenz) Infpectoris Mufei rer, nat, \& art. Reg. Dan. Havoienf. tres tabilæ anex cum iconibus teftaceorum partim rariffimorum, iu folio. 
fic etiam Lifterus 7), Adanfon 8), Chent- fo haben avch Lifier 7), Adanfon 8), uitius 9), Da Cofta 10), Du Chesne 1 1), Chemnitz 9), Da Cofta Io), Du Chesue pauca publicaverunt, fic etiam Bafchius, 11 ), fehr wenige; ingleichen Batfch, der obfervator, fimulque pictor \& fculptor, als Beobachter, Mahler und Kufferffecher quod rarum contingens, interim fex fo- zugleich, welches ein feltner Fall if, mit lum fpeciebus initium fecit 12).

6 Arten einfreveilen den Anfang genacht hat 12).

Alii auctores: Gualtierus, LedermiilAntere Schriftfeller, als Gualtieri, lerus, Martini, Actorum Societatis Gedi- I.cdermiiller, Martini, der Danziger Genenfis \&c. potifimum folas tantum co- fellfchaftl. Abhandlungen \&ंc. haben gröjspias ex aliis feriptoribus \& paucinima ten Theils nur Copien ans andern Autoren, propria publicarunt, ita ut huc adferri haud rite poffint.

dagegen aber fo wenig eigenes, dafs man fie nicht wohl hierher rechnen kann.

Plurima autem a prædictis auctoribus repræfentata conchylia folum ad minora, quorum longitudo aliquot linearum eft,

Die mciften in diefen Schriftftellern abgebildeten Conchylien gehören aber nur su den kleinern, deren Länge etliche Li-

Ejusd. Prælectiones in Actis Reg. Dan. Societatis fcientiarum Havn. cum tab. æneis, $\&$

Ejusd. Tabulæ interim æri incifæ ad Catalogum defideratiffimum Teftaceorum fuorum.

7) Lifter (Mart.) Hiftoria I. Synopfis Conchyliorum Libri IV. Londini edit. I. I6 55 I6g6. Fol. Edit, II. per Guil. Huddesford. Oxoniæ 1770. Fol, tabulæ æri incifæ.

8) Hiftoire naturelle du Senegal. Coquillages; par Mr. Alanfon. Paris 7757 . 4to. avec figures.

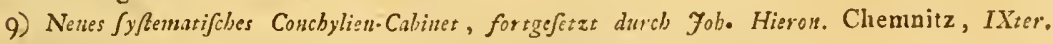
Baud, mit nach der Natur gemablten Kupferm. Nimberg 1786. 4to.

10) Hiftoria Naturalis Teftaceorum Britanuix, or the Britilh Conchology \&c. ou la Conchologie Britannique \&c. Cum fig. coloratis; par Em. Mendez $\mathrm{Da}$ Cofta. London 1778. 4 .

11) Recueil des Coquilles fluviatiles \& terrefires, qui fe trouvent aux environs de Paris, deflinées, gravées \& enluminées d'après nature par $D u$ Clesue \&c. fuivant l'ordre, que leur a donnée Mr. Geofroy. Paris (fine anno) 4. 3 Tab.

12) Sechs Kupfertafeln mit Conclyliell des Seefandes, gezeichust und geffochen von A. F. G. C. Batich, Fens I79I, 4. 
pertinent; pauca ad minima proprie microfcopica denominanda, de his præaliis Soldanius optime meruit.

Ad remedendum itaque, quantum poteft, huic naturalis hiftorize defectui duo lueic Vindobonæ degentes fcicntiæ lujus cultores, partim proprio impulfu, partim aliorum compellatione excitati, ante circiter quatuor annos induxerunt animum coadunatis viribus fefe accingendi operi \& fccundum omnia, qua comparari poterunt, originalia omnique diligentia hanc materiam elaborandi atque iconcs æri incifas beneque coloratas cum defcriptionibus plenis, neque tamen rerbofis, in publicum edendi. Ex liac conjunctione redundabat, ut jam 500 icones totidem fpecierum vel partin varietatum, quarum qualibet primo loco ma-. gnitudine naturali, $\&$ tunc microfcopio valde aucta rel ab umo, vel duobus, fapius etiam pluribus lateribus, quædam etiam, fi fcopo conducebat, fecundum diverfas fectiones delineatre, omnes autem, non ex aliis operibus mutuata, fed fecundum tot originalia, qua unus horum amicorum, adhibitis obferwationibus alterius, microfcopiorum bonorum ope laboriofifime \& accuratiffime ipfemet nien hält; wenige au den kleinflen eigentlich mikrefhopifchen; um dieje letztern hat voraighlich Soldani das meifte $V$ erdieuft.

Un mun diefe Lücke in der Naturgefinichte foviel möglich ausaufüllen, haben arey hier in Wien lebende Liebhaber der Naturgefchichte, theils ans eigenen Antriebe, theils durch $Z$ upprechen anderer aufgenuntert, fich rar ungefihr vier Falwen vereinigt, diefes Fach mit aller Enfiglieit, nach allen nur möglichft au bekomnenden Originalen su bearbeiten und daron in Kupfer geftochene und gut illuninirte Abbildungen fonnut unfä̈ndlichen, doith aber nicht weitlunftigen, Beformeibungen heraus su gebers. Das Refultat diefer Verbindung war , dafs jetzt lereits 500 Abbildun; en von fo riel Arten oder ann Theil Abcinderungen, deren jede cin Mahl in natiirlicher Gröfse und fodann fark vergrü/sert von ciner, swey und üfters mehreven Seiten, einige auch, wena man es für sweskmajsigh hielt, mash zerfohiedenen Durchfohnitten, aler nicht aus andern Werken entlehnet, fondern alle nach lauter Originalen genuacht find, die einer diefer Freunde nit ier. cinigten Beohaihtungen des andern, nit Hiilfe guter Mikrofkope, auf das mühe- 
delineavit eorumque coloribus propriis pinxit, cum defcriptionibus latinis \& germanicis paratæ adfunt. Hædefcriptiones in lingua technica Linnæi claborata, regulis ejus in Philofoplia botanica, ab ipfo datis, quantum fuit pollibile, firicte adhibitis. Hac occafione aliquot nova detecta funt genera, multæque novæ partim rarifimæe fpecies, fimulque, non novationis pruritu, fed urgente attentitiore contemplatione aptum partimque neceffarium vifum eft, ut aliquales in Syftemate Limmano fierent mutationes; fic ex. gr. genus Linnaanum Nautius dictum, folummodo propter multos thalamos, poftlabito omnium reliquarum partium diffenfu facieque externa $f$, habitu, nimis in unum confipatum, in quatuor genera, nimirum Noutilus, Homtmonium, Orthoceras \& Lituus divifum eft 13), porro Argonauta Cymbizm Linn. ad polythalamia translatus \&c. Pro objectis non folum Teftacea microfcopica proprie fic dicta electa funt, fed etiam

13) Hac in re jam anteceforem habemus, in hac naturalis hiftoriæ parte pro ifto tempore, quo vixit, faniori doctrina iofructum Breyniuziz, qui in fua Differt. foylo de Pobrialamiis Éc. Gedani 1732. 4. 7. 12. eaden divifone, ordine Solum inverfo, i. $\mathrm{us} \mathrm{c}$. $\mathrm{s}$. fanfte fellift gezeichnet und mit ihren natiirlichen Farben gemalilet hat, faimmt den dazu gehörigen lateinifch-und dentfchen Befchreibungen, fertig da liegen. Die Befchreibungen find in der won Linné eingefuibrten Kunftprache abgefafet, wo man möglichft feine in der Philofophia lotanica gegebenen Regeln befolget hat. Hierbey haben fich etliche neue Gefchlech. ter und viele neue aum Theil Selir feltene Arten entdecket; anch fand man, wicht mus N'enernngssucht, fondern durch die genaure Beobachtung veranlafet, fiir. fchicklich und theils nüthig, in dem linneifchen Syften einige Abänderungen su treffen, ə. B. aus Limués einzigen, blofs wegen der vielen Kanumern mit Hintanfetanng aller übrigen Unubereinftinumung der Theile und des äufserlichon Anfehesss su Jehr sufanumen gesunngenen Gefohlecht Nautilus vier Gefchlechter: Nautilus, Hammonium, Orthoceras und Lituus, zu machen 13), den Argonauta Cymbium L. unter die vielkammerigen $\approx u$ verjetzen

13) Wir baben bierin berents cinen Vorgänger ail den in dieftn Facb der Naturgefcbichte fiir $\int$ in Zeitalter felor anfgeklärsen Breyn, dir in finer. Diflert. phyf. de Polythala. miis \&c. Gedani 1732. 4.p. 12. eben diefe Eintheilung, nur in ungemandter Ordnung bat. 
alia minuta maguitudinis àliquot linca. rum usque ad climidium pollicem \& quod excurrit, quæ Batavis nomine Sperulaz jes venimint. Ut antem Syficma reddatur completius, forfilia diftincta non exclufa funt, qua in globi noftri tcraquei hiftoria fane magnum prabent ufum. Ex. gr. pluxes regiones montanæ ab inundationibus marinis antiquis ex ca folimmodo ratione adhucdum liberae ccufita funt, quia nulla majoris molis tefiacea olim marina numc fofilia ibi inveniuntur, cum tamen perquifitione microfcopica cjusmodi corporibus minutis fape tota repleta offenditur terra. Sic teftimonio Dni Abbatis Soldani : 4) nomulli dubitarunt, 2n prealli Apcuniui montes in Thuscia ex aquis marinis originem duxcrint, quia nulla conchyliorum folfilium ibi apparent veftigia. Af ommes non nif oculo fugiti. vo grandiora quærebant teftacea, minutia non animadvertebant, quia nen manibus, fed oculo aquilino, frepins folum armato palpabilia futit, quorum tamen multis in locis, ctiam editifimis, maxima adeft copia, \& aliquando integra apparent fse, die ganz kleinen ïberfahe man, weil
Arata lapideo-calcaria, aut arenaria, fre nicht mit Händen, fondern mit fchar-
conchyliis minimis aliisque productis fem, ofters nur bewaffnetem Auge fafscopia, \& aliquando integra apparent fse, die ganz kleinen ïberfahe man, weil
Arata lapideo-calcaria, aut arenaria, fre nicht mit Händen, fondern mit fchar-
conchyliis minimis aliisque productis fem, ofters nur bewaffnetem Auge fafscopia, \& aliquando integra apparent fse, die ganz kleinen ïberfahe man, weil
Arata lapideo-calcaria, aut arenaria, fre nicht mit Händen, fondern mit fchar-
conchyliis minimis aliisque productis fem, ofters nur bewaffnetem Auge fafs-

14) Soldani fupra alleg. Teftaceographia Tom. I. Prolegom. p. 11. Iq. confer. Ejusd. Sazgio orittografico p. 84. 5:57. - p. 89 .

u. $\int f . Z_{i l}$ Gegenfänden hat man nicht mur die mitrnfropifichen Convihylien in eigentlichun Verffundte gewaih't, fondern unch diejenigen zon der Gröfse etlicher Linien bis etwas uler einen hirlben Zoll, die von den Hollcinchn Speculazjes genannt werden. Mans hat aber, un das Syftem collftindiger su mocben, auch deutlichic forfule nicht ansgefchloffen, welche in der Gefotichice uajers Er dlalles ge-

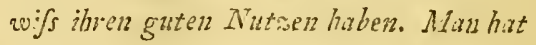
¿. B. bisher manche Gebirgsgegenden defswegen fïr frey zon elienahligen Meeresïberfohvemmungen gehalten, weil man keine grüjsern fofflen Mecr - Schalthiere dayelbft antrifft, da man doch bey mikrokopiforer Unterfuchung das Er dreich woll zon dergleichen kleinen Meertiörpinn findet. So wurde uach dem Zeugniffe des Horm Abts Soldani It? gesse eifelt, ob das fehr hohe Apenninifiche Gebirg in Toscana Seiven Unfpring im Meer gehorbt bate, weil mon heine Spu* :0n foffilen Meer-Schalthieren darauf fond. Marizuch. te aber auch nur mit fiichtigem Auge grocopia, \& aliquando integra apparent fse, die ganz kleinen ïberfahe man, weil
frata lapideo-calcaria, aut arenaria, fen nicht mit Händen, fondern mit fchar-
conchyliis minimis aliisque productis fem, ofters nur bewaffneten Auge fafs-

\section{exaquis marinis originems chuxcrint, quia} Abbatis Soldani t4) nomulli dubitarunt, to palpabilia finte, guorum tamen multis to 8 
olim marinis partim referta,

lich find, und die an wielen, wuch dew hüchften Orten, in gro/ser Menge liegen, auch marchmahl gañe Kalhftein- und Sondfinchen mit den kleinften Conchylien und andern ehemahligen Mecrkörperna sitn Theil angefillet find.

Laboriof hujus operis primitiz nunc Voin diefer Arbeit erjcheint nun das in lucem prodeunt, in quibus ex Linnzano genere Airgonunta \& noftro Noutilus dicto, yuod germanice nobis Sihiffer audit \& parten folummodo Nantili Linnaani comprehendit, fpecies minutx \& microf́copica tractantur. Cpusculum hoc vel pro Nionographio feli Tracta. tu folitario de hac conclyyliologia parte putari, vel pro appendice feu fupplemento Opernon conchyliologicorum a Marti-: nio \& Chemnitio editorum haberi poterit.

Nomina fpecifica, que jam in aliis fcriptis exfabant $\&$ adiequata erant, rete:ıta funt; nova, quorum longe maxima pars eft, potifimum a fimilitudine formi externæ, quatenus fieri potuit, depromta funt, ex yuibus forte nonnulla auribus delicatioribus non admodum placitur?. Quisquis autem aquanimus lector, qui fcit, quam difficilis ad fatis a fe invicem diftinguendas fpecies in hilitoria jeder billiger Lefer, welcher weifs, wie naturali, prefertim in amplis gencribus, Jchuer es fey, in der Noturgefchichte, lefit novorum nominum, brevium \& adæ- foudersin itcitlälftigen Gefchlchteru, $\approx$ is 
quatorum, creatio, fi non pro unico no- hinlcinglicher Unterfoheidung der Arten mine, uti multorun mos eft, ex pluri- zon einander, nene, kurze und angemefbus vocabulis compofitas adhibere volu- Sene Nurhnen $\approx n$ fchöpfen, wemn nun nicht, mus defcriptiones, nobis facile indul- anfatt eines einaigen Nahmens, wie viegebit.

le im Gebrauche haben, aus ctlichen Wörtern anfannuen gefetate Befohreibungen machen will, wird leicht mit uns NachSicht haben.

Si forte ex voto nofiro fufficiens em-

Sollte fich, anferem Wunfche gemäß, torum aderit numerus, quo impenfe eine hinlängliche Ansahl Abnehmer finden, editionis fartæ tectæque mancant, inten- da/s die Koflen der Anflage einigermafsen tio eft, tali modo editioncm phurium gedechtwiirden, fo ift man gefonnen, auf ejusmodi tractatuum tamdiu continuan- diefe Art mit mehrem Abhandlungen fo di, usquedum non modo omnia a Lin. lange fortaufahren, , bis nicht nur alle næo propofita genera abfoluta, fed eti- von Linné aufgefteliten Gefihlechter durch. am aliquot nova pertractata erunt, quem in finem fat magua fpeciminum copia ad gegangen, fondern auch aerfchiedene neue abgehandelt feyn worden, wosu bereits manus eft, \& cujus augmentationem a ziemlich Vorrath vorhanden ift, deffen benevolentia fautorum amicorumque, Vermehrung wir von der theilnelhmenquibus fudium conchyliologicum cordi den Giite conchyliologifcher Gönner und eft, ad meliorem ejus promotionem \& Freunde aur Beförderung und Erweitepropagationem exoptamus cosque, offi- rung diefer Kenntniffe wïnfchen und mit cia noftra reciproca offerendo paralifi- Anbietung bereitwilligfler Gegendienfte ma, enixe obteliamur. darum bitten. 


\section{I $N$ N D \\ T A B U L A R U M.}

TAB. 1, Fig. a-e. Argonauta Cornu, $W_{\text {idderhorn - Argonahte. }}$

- 2. - a. b. c. Nautilus Pompi- Der kleine dicke Sshiffer. lioides.

- - - d.e.f. Nautilus Vortex. Wirbelfihiffer.

- - - g. h. $i_{.}$- - dus. dimidia. - - angefchliffe\%.

- 3. - a.b.c.d. Nautilus repan- Der ausgefinweifte Schiffer. dus,

- - - e-h. Nautilus afterizans. Der geffirnte Sciniffer.

- 4. - a.b.c. Nautilus incraff- Der aufgeblafene Schiffer.

tus.

- .. - d.e.f. Nautilus crifpus. Der kraufe Schiffer.

- -. - g.h. i. Nantilus coltatus. Der geribbte Schiffer.

- 5. - $a_{0}$ b. Nautilus crifpus di- Der kraule Schiffer aufgefichlifen. midiatus.

- -- c:-g. Nautilus ftrigilatus, Der gefricholte Schiffer 1. und 2. AbänVar, $\alpha . \& \beta$. derung.

- - - h.i.k. Nautilus craticula- Der geflochtene Schiffer. tus.

- 6. - a.b.d. Nautilus Mammil. Der Bruftuarzeiz-Schiffer. $1 a$. 


\section{I $\quad \mathrm{N} \quad \mathrm{D} \quad \mathrm{E} \quad \mathrm{X}$}

TAB.6. Fig. G. Nantilus Mammilla di- Der Bruftsomren-Schiffer aufgefshliffen. midiatus.

- .- - e.f. h. Naut. lenticularis, Der Linfenförmige Sihifer, 1. Alü̈nt. - Var. a.

- - - g. Naut. lentic, dimidiatus. $\quad$ - - aufgefchliffen.

- 7. - a.b. Naut, lent. Var. B. - - _ - 2. Abind.

- - - c. d. - Var $\gamma$ - - - 3. Abiard.

- - - e.f. - - dimid. - - - aufgesibliffeit.

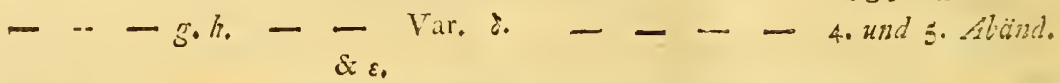

- S. - a. b. d. Nautilus radia- Der geftralite Schiffer. tus,

- - - c. - dimidiatus. - - - alfgefuhliffert.

- - - e.f. h. Nautilus venofus. Der geäderte Schiffer.

- -. - g. - dimidiatus. - - - anfgefohliffen.

- 9. - a. b. c. Naut. firiato-punc- Der geftreift-punctirte Schiffer: tatus.

- -- - d. e.f. Naut, ambiguus. Der sweydeutige Schiffer.

- -. - s. h. i. - farctus, Der cusgeffopfte Sshiffer.

- 10. $-a-d$. - fiutuatus. Der buchtige Schiffer.

- -. - $-e^{-k}$ - macellus, Der magere Sehiffer, 1 und 2. Abünd. Var. $\alpha$. \& $\beta$.

- iI. - a-k. Naut. Calcar. Var, Der Sporn-Schiffer, i-4. Abänd, . $\alpha-\delta$.

- 12. - a-k. - - Var. - - - $5-8$.

- 13. - a-i. - - Var. - - - 9-12. 1. $-\mu$.

$-\quad-k .0-\frac{\text { dimi- }}{\text { diatus. }}-$ aufgefchliffer. 


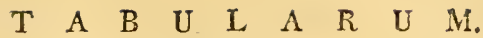

TAB. 14. Fig a. b.c. Naut. papillofus, Der vieharaige Sihffer.

- - - d-i. Nautilus Spengleri, Der Spenglerifre Schiffer, 1. und 2. Var. $\alpha, \& \beta$,

Alïinderuns.

- 15. - a-h. - - - - $-3.4 .4 .5 . A b a i z d$.

- - - - - - - - - dimidiatus. - anfgelihiffen.

- 16. - a-h. Nautilus planatus, Der fache Sihifer, 1. 2. 4. 3. Abcind.

$$
\text { Var, «. ß. } \gamma \text {. }
$$

- - - i. - dimidiatus. - - - aufgefchliffin.

- 17. - a-1. Naut. Callis, Var. Derhelmformige Schiffer., I-4. Abänd

$$
a-8 \text {. }
$$

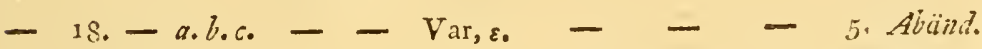

- -. - d. e.f. Naut, Galea, Der Aurmhabenformige Schiffir.

- .. - g. h. i. Naut, acutauricu- Der Spitzohrfehiffer. laris,

- 19. - a. b. c. Naut. Faba. Der bohnenfürmige Schiffer.

- -- - d. e.f. Naut. Scapha. Der Kahufiniffer.

- -- - g.h. i. Naut, Crepidula. Der pantoffelähnliche Schiffer.

- 20. - a-f. Naut. Auricula. Var. Der ohrförmige Sihiffer, 1, u. 2. Alünd. a. \& $\beta$.

- -- - g-k. Naut. tuberofus. Der knollige Schiffer.

- 21. - a. b. d. Naut. Orbicu- Der fcheibenfurmige Schiffer. lus.

- - - - c. - dimidiatus. - - aufgefchliffer.

- 22. - a. b. c. d. Nant, angula- Der winlelige Schiffer. tus.

- - - e. - dimidiatus. - - - aufgefohiffen. 
I N D E X T A B U L A R U M.

TAB, 23. Fig. a. b. d. e. Naut, adun- Der eingekrümmte Schiffer. cus.

- - - 6. - dimidiatus. - - - halbaufgefchliffer.

- 24. - a.b. c. d. e. Naut. Melo. Der Melonen-Schiffer, 1. Abänd. Var. $\alpha$.

- - - f. - dimidiatus. - - - halbaufgefchliffen.

- - - g.h. - Var. 6 . - 2. Abänd. 


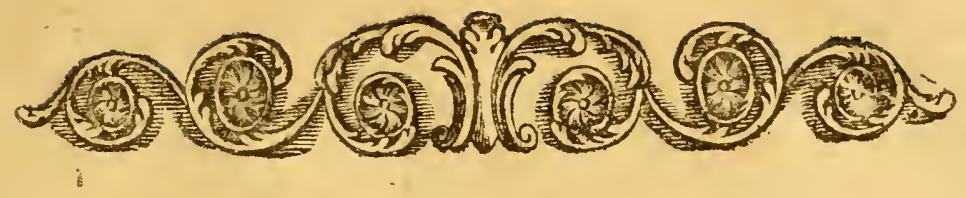

\section{ARGONAUTA.}

TESTA univalvis, fpiralis, vel involuta, vel convoluta, cymbiformis, unilocularis;

ANFRACTUS vel obtecti, vel aperti, comprefli, carinati ;

APERTURA magna, dilatata.

Ab omnibus veteribus hiftoriæ naturalis fcriptoribus, eo tempore, quo e linnæano genere Nautilus dicto unicam folum fpeciem, fcil. N. Pompilium, \& ex obvio genere nonnifi Argonautam Argo noverant, etiam a multis recentioribus, imo a D. Martini ipfo a), cui tamen jam aliquot fpecies notæ erant, quas autem pro meris varietatibus habuit, hocce geuus folummodo ut fpecies refpiciebatur, atque cum ifto Nautilo Pompilio tan-

9) Syftemat, Conchyl, Cab, I, I, p. Igs, feqq.

\section{ARGONAUTE.}

Die SCHALE beftebt aus eirem Sticke, ift fchlirkel-(Spiral-) förmig, entweder eingcrollt, oder zufansmengerollt, nacbenformig, einkamnicrig; die

WINDUNGEN find enswoder bedeckt, oder alle facbrbar, zufammen gedrickt, gekielet; die $M \ddot{U N D U N G}$ ift grufs und ermeitert.

$V_{012}$ turgefchichte wurde damohls, da now arus deun linneifchen Gefchlechte Nautilus nur eine einsige Art, den Naut. Ponpilius, und aus gegenwörtigen bloss den Argonanta Argo kannte, auch von vielen neuern, ja fogar vonn fel. Martini jelbft ${ }^{2}$ ), den doch bereits ctli he Arten bekannt waren, die er aber als bloßse Abänderungen behardelte, diefes Gefchlecht bloßs als eine Ait angefeben, und mit ge-

2) Syfenat. Conshylicukabinet I. Banil, S. Igs. ff. 
quam altera fpecie in unum utrisque commune conjungebatur genus, cui imponebant varia nomina: Nautilus, Nauplius, Pompilius, Nautes, Navicula \&c. belgice: Zeiler, Schipper \&c. germ. Segler, Schiffer, Schiffkuttel, Schiffboot \&c. ambasque fic dictas fpecies folummodo in tenues \& craflas diftinguebant, ac prio. rem Nautilum tenuem, Cymbium; belg. Doekehouve, fne Schippertje, Zeiler; germ. diunnes Schiffboot, geribbte Galee. re, holländifche oder Kammertuchhaube, Papjer-Nautilus, papierne Segler \&c. nominabant; pofteriorem vero Nautilum craffum, Naut. concameratum \&c. belg. Parl-Slakke, Parlemoerhoorn, Kocquilie, Schipper \&c. germ. dickfchaligen Schiffkuttel oder Schiffboot mit Kammern, Schiffchen, Schiffer, Perlfchnecke \&c. b).

Solus ex recentioribus Linnaus, prop. ter magnam effentialemque inter MONO. THALAHIUM \& POLYTHALAMIUM differentiam, duo exinde conftituebat genera, ac uniloculari nomen ARGONAU$\mathrm{T} \mathbb{\mathrm { E }}$, multiloculari vero NAUTILI imponebat. Quo melius quacunque in pofterum evitetur amphibolia, celeberri-

b) Frequentiores folunmodo denominaliones alIegamus; plures inveniuntur in Martini Concb. Cab. T. I. p. I99-205, ubi etiam gallica, aliaque exflant nomina. dachten Naut. Ponpilius als der anderra Art in ein einsiges beyden fo genannten Arten gemeinf:haftliches Gefchleht vereiniget, welchem fie verfshiedene Nahmenz beylegten, als: Lat. Nautilus, Nuuplius, Pompilius, Nautes, Navicula etc. Iroll. Zeiler, Schipper etc. deutfch: Segler, Schiffer, Schiffkuttel, Sinhffloot Vco und beyde fogenannte Arten in diunn-und dickJchalige unterfchieden, und die erfern Nrutilum tenuen, Cymbium; holländ. Doekelourte, fune Schippertje, Zeiler; deutich: dïnnes Sihiffboot, geribbte $G a-$ leeve, holländifche oder Kammertuchhaube, Papiernautilus, papiernen Segler $\Im$ c. nanziten, die letatere Art aber unter der Benennung: Nantilus craflus, Nautilus concameratus etc, holl. Parrl-Slakke, Par. lemoerhoorn, Kocquilie, Schipper etc: deutfich: dickffhaliger Schiffkuttel, oder Schiffloot mit Kammeru, Schiffchen, Sclriffer, Perlfchnecke Evc. auffiihrten b). Nur Linné war aus den neuern der einsige, der wegen des grofsen und wefentlichen Unterfohiedes awifichen EINKAMMERIG HiZd ZWEYKAMMERIG swey eigene Gefchlechter daraus machte, und das einficherige mit den Nahmenz Argonanta, das vielfâtherige hingeger mit Nautilus belegte. Un alle mögliche

b) Wir fulbenz hier n:r die gewöbnlichften Benennungen an; mebrere findet man in Martini Conchylienkabinet I. Band S. $199^{-20} 5$, wo auch die franzofrtaen und azderer spracben Nabmen befinlicicio finsl. 
mum virum nos hic fequimur, etiam quoad denominationem germanicam eousque, ut genus prius ARGONAUTE a latino, pofterius autem SCHIFFER tanquam latinum fignificaum Nautilus, tam intuitu animalis, quam tefæx, præ aliis potifimum exprimens vocemus, Voca. bulum ScHIFFBоOт non adamavimus, quia nimis limitatum \& magis fpeciale, quam genericum nobis videtur. Vocem KAMMERSCHNECKE (i. e. Cochlea con-. camerata, f. polythalamia) qua D. a Born c) in denominando toto linneano genere Nautilus dicto ufus eft, lubenter adoptafemus. Cum vero hoc unicum genus linneanum in quatuor diverfa difpescuerimus genera , Nantilum nempe, Hantmonium, Lituun \& Orthoceras (vid. Introd. noftr. ad gen. Nontilus), \& hac omnia polythalamia fint, vocabulum iftud in fenfu rigorofo neutiquam fuifet adæquatum nomen genericum.

Intuitu nexus fy ftematici inter genera concipere nondum polfumus, quare Linnæus ab ordine, quem in collocandis \& defcribendis naturalibus fibi aliisque præ. fcripferat $d$ ) \& ipfemet fecutus eft, a per-

c) Ind. Mur, Caer. Vindob. p. 121. B.

d) Philofoph, botan, 1751, P. 97. §. 153 .
Zweydeutigkeit für die Zukunft su ver" meiden, folgen wir hier diefen berihnten Manne auch in der deatfchen Bencnming in fofern, daß wir das erfiere Gefchlecht nach den Lateinifshen A $\mathrm{kgONAU}$ TE nennen, und denu letatern den Nirnmen ScHIFFER, welches die Bedeutung des lateinifchen Worts N'autilus, fouohl in Anfehnng des Einwohners als der Sthale, vor anden vorwinglich ausdrückt, beylegen, Das Wort ScHIF FBоO T gefiel uns defswegen nicht recht, weil es $\approx u$ eingefchrainkt und nehr auf eine Art, als auf ein Gefshlecht, pafsend su Jeyn fohien. Hizgegerz den Nahmen KA Mu R S CH N ECKE $\left(d\right.$. i. Cochlea concamerata $\int$. polythalamia), deffen fich $H r, v$, Bornc) $z u$ Benennung des gansen linueifchen $\mathrm{Ge}$ fohle:hts Nautilus bediente, hatten wir gem angenommen. Weil wir aber diefes einaige linneifche Gefchlecht in sier serfchiedene Gefchlechter, nehml. Nautilus, Hammonium, Lituus $u$ : Orthoceras zer. theilet haben, (frehe unfere Einleitung amm Gefchlechte Nautilus, ) und diefe alle vielkammerig find, fo wiirde diefes Wort fcharf genommen keineswegs ein angemeffener Gefchlechtsnahme gewefen Jeyn.

In Anjehang des fyftematifchen $Z u$ fanmenhangs der Gefollecinter unter einander können wir noch nicht einjehen, warum Linnet von der Ordnung, die er in Aneinanderreihnng und befclireilung

c) Lzdex Muf. Caf, Vindob, p, I21. R. 
fectioribus nimirum \& magis compofitis ad minus perfecta \& fimpliciora progrediendi, hic declinarit, \& transeundo ab animalibus bivalvibus ad univalvia, Argonantam tefta fimpliciori donatum alitepofuerit Nautilo multo magis compofito. D. a Born quidem hanc aberrationem excufare tentavit e) verbis: , nec, tit quafi Limnes teftas univalves cum , bivalvibus, dum Pinnæ poftremum ", inter conchas, Argonante vero pri" mum inter Cochleas locum afignat. "Quemadmodum enim e valvis binis , in unam prope teftam coalitis compo„, nitur Pinna, ita \& Argonauta unival„, vis e tribus fere partibus, ut recte ex", plicat Bellonius, in unam teftam com", pactus eft, nempe e lateribus duobus ", \& carina, ita ut Cymbii figuram ", perfecte fatis referat. "Sed ratio hæc nobis haud fatisfecit ad repellendam fententiam nofiram, Limæum legi fuæ hic minus accurate fequacem fuife. Etenim fi partes, quibus hæc vel illa tefta compofita eft, hoc modo numerare volumus, fane in Nautilo majorem partium numerum conferre poflumus, quo facto hoc genus femper magis compolitum illo, fcilicet Argonautarum genere, evafurum, eique in adoptata ferie antecedere neceffe erit.

\footnotetext{
e) Muf. Caef. Vindob. Teftac. fol, p. $1 \hat{\jmath} 8$.
}

natürlicher Dinge fich und andern vor. gefolrieben hatte d), wuch ihr felbft gefolgt ift, nehnlich von den vollhommenem und sufanmeingefetatcrn, au den minder vollkommenern oder einfochern fortzufchreiten, hier abgewichen, und bey feinem Uebergange von den sweyfchaligen Thieren au den einfchaligen den mit einer cinfachern Schale begabten Argonauten feinem mit einer weit mehr sufammengefetaten Schale verfehenen Noutilus vorgefetst habe. Herr v. Bornfuchte zwar diefe Abweichung au entfchuldigen '), wenn er fagt: , Inden Linmé den Steck"mufcheln die letate Stelle unter den "Mufcheln und den Argonauten die er", fte unter den Schnecken einraunet, fo "verbindet er auf gewiffe Weife die ein"Jchaligen Schrithieve nit den aweyklap"pigen. Denn gleichwie die Stechmuf chel ", alls zwey faft in eine Schale sufammen "gesuachfenen Kilappen beftehet, fo ift ", auch der einfchalige Argonaute bey. "nahe aus drey Theilen, wie Billonius "wohl evinuert, in eine Schale zufam, mengefetst, nehnlich aus 2 Seiten und " denn ríei, fo daßs er eine siemlich "Schiffulhnlishe Geftalt hat." Allein diefes ift uns nicht uberreugend genug, um uns von der obgefagten Meinuing abzu. Uringen, daf Linzể hier feiner Regel nicht genou gefolgt Jey; denn wenn man die Theile, woraus eine Schale sufammen ge.

d) Phil. botan, 1751, p. 97. S. 153 .

e) Noxf. Caref. Viridok. Tefisco fol, fo 138 . 
Genus ARgONAUt A fpeciebus pauperrimum eft, fecundum Linnæi fyftema naturæ noviflimæ edit. XIII. Gmelinianæ nonnifi quinque, Argonautam ARgo, vitreum, Cymbium, Cornu \& ARCTICUM, continens. Speciei fecundæ rariffima (A. vitreus) ${ }^{f}$ ) unicum hucusque repertum, ab Argenvillio obiter defcriptum fpecimen nobis femper fufpectum fuit, an revera fit tale, pro quo venditur, \& annon fit polythalamium; at numc ex altero nuper noto fpecimine s) certiores facti fumus, quod plane nullis gaudeat thalamis \& hoc refpectu ad hocce pertineat genus. Quod attinet ad

f) Olum in Mufeo D. Lionet Haga Comitum vid. Argenville Conchyl. Append. Tab. 1. fig. B. verf. germ. 1772. Tab. 10. Fig. B. ex họ̣ in Miartini Conchyl. Cab, Bd. I. p. $2 \xi 9$, Tab, 18. Fig. 163 . translat.

ह) Specimen iflud fecundum ex mufeo Dni, Car lonne, gallici olim ftatus adminifri Londini degentis, in manus Angli cujusdam venditione perventum alter noftrum amborum in elaborando hoc libello confortium nuperrime cx itinere in Angliam facto redux (Leopoldus a Fichtel) iteratis vicibus omni oum cautione \& diligentia infpexit \& pro vero monothalamio agnevit; fed, utrum proprie teftaceum, an aliud animalis cujuscuuque marini pars fit, in dubio eft.
Setot if, wuf folche Art wihlen will, fo wird man an einem Nautilus noch mehreve heraus bringen, diefer folglich immer sufanmen gefetater Seyn, als der Argonaute, und ilin in der angenominenen Reihe vorgehen minffen.

Dirs Gejchlecht Argonaute enthailt felir wenig Arten, nach der neneAen XIII. Gmelinifchen Ansgabe des linnäifshen Naturfyftens nicht mehr als fünf; diefe find der ARGONAU TA ARGO, VItreus, Cymbiun, Cornu und ar. c TICus, Das von der zweyten feltenffen Art (A. vitreus) ) bisher erft einmanhl angetroffene, rom Argenville ober fichlich befitiriebene Stück war uns imner verdachtig, ob es wirklich das wäre, wofur es ausgegeben wird, und ob es nicht etwa vielfacherig fey; allein jetat find wir durch ein anveytes bekanint gecwordenzes Stitik B) vergewiffert voorden,

f) Diefs bcfond ficb in dem ebemabligen Kabinet des Hry. Lionnet in Hang; fiebe Argenville Concbyliologie Append. Tab. 1. fig. B. dentliber Ueberfetzung $177^{2}$. Taf. 10, fig. B. und bieraus won Martini in fein Conch. Kab. Bd. I. S. $239 . T_{a f}, 18, f_{g} .163$. übergetragen.

g) Obigges zweytes Strïck uns der Sammlung des in London lebenden ehemabligen franzïfifcben Stautsminifters, Hrn. Calonne, das durch Kauf in den Befutz eines gewijien Englänlers kam, bat der eine von uns beyden zur Ausabeitung diefes Werks verburdenten unling $A$ von finer nach Englaud gemachten. Reife aurück, gekommene (Leopold von Fichtel) vejfbicitene Mahle mit aller Vorficht uni Fleifs betrichtet, und fur ein wallres einfacheriges Stück arterknnnt, aber, $O b$ cs ein eigentliches Scbattbiergebuufe, oiler ein Theil 
fpeciem quintam (A.arcticus), etiam nunc adhuc ut incompertum in medio relinquimus; an hujus tribus fit, nec ne. Figura ad minimum \& denominatio a D. Ott. Fabricio in Fauna Grönlandica p. 386.11.384. ex Blefkenii itiuere (Adelung Gefchichte der Schiffarten \&c. p. 410. tab. 17. fig.12.) huc allegatre ipfaque Fabricii defcriptio hanc fpeciem hinc ad aliud genus, forte ad Helices transferre nos jubent. De fpecie tertia ARG. Cy мвrum dicta, a Linnæo huc relata, autoplia fpeciminum, non arenæ folım magnitudine, ficut vulgo venditatur, fed unius duarumque linearum longitudine etiam diffractorum plene convicti fumus, eam evidenter poJythalamiam effe, uti omues confiteri debent, quibus non folam teftæ ftructt:ram externam, fed precipue internam intueri libet; quapropter etiam hanc fpeciem ad alind nimirum polythalamium genus remifimus. Species quarta, ArG. CORNu, an legitimum hic occupet locum, vel potius alii generi inferenda fit, adluc clubii hæremus, (vid. Defcript. noftra fpecifica.) Sic detractis detrahendis vix duæ tresve huic generi permanebunt veræ fpecies, \& fortaflis firictiori fenfu fola fpecies prima, ARG. Argo, \& fecunda, A. vitreus; nifi inter omnia ifta hucusque nota fub varietatum nonsine in hanc fpeciem contracta fpecimina forte aliquot, non cha- dafs es auf keine Weife Krmmern habe, und in diefenn Bctrachte su diefen Gefchlechte gehöre. In -Anfehung der fünften Art (A. arcticus) laffen wir es auch noch dahin geftellt Jeyn, ob fie hierhier au rechnen fey, oder nicht. Wenigftens die von Hern Otto Fabricius in feiner grözldindifchen Faune aus Blefkens Reife (Adelungs Gefchichte der Schiffaiten $\mathcal{E}^{\circ}$. S. 410. Tiafel 17. fig. 12.) hieher citirte Abbildung und Benzennung, ja fellof die Befchreibung des Fabricius heißen uns diefe Art von hier weg in ein anderes Gefohlecht, vielleidht au den Schnirkelfchnecken, ubersutragen. Von der dritten von Linne hicher geaogenen A. CYMBIUM genannten Art find wir durch eigene Betra:htung folcher Stuicke, die nicht bloßs zon Srndkorngrößse, wie fie insgenein ausgegeben werden, fondern von einer bis awey Linien in der Lange, auch entswey gebrochen find, vollkommez uberfuhret, das fie offenbar vielkannerig ift, wie alle diejenigen eingeftehen miuffen, denen es belieben wird, nicht blofs den äufern Bau der. Schule, fondernz hanptsichlich den imnern einsufehen, wefswegen wir auch diefe Ait in ein anderes und zwar vielficheriges Gefchlecht übergetragen haben. Ol die vierte Art, A. Consu; hier an rechten Orte flehe, oder vielnehr einem andern Gefshlechte einzuverleiben fey, darüber ftchen wis.

irgend eines andern seetbieres fey, das if ibur nocis aweifelh oft. 
sactere variabili, fed conftante fat di- noch in Zweifelo (Siehe herrach die Beverfa, duas vel tres confituere poterunt fchreibung diefer. Art). Wird folchemfpecies.

nach dasjenige weggenommen, was vor Rechtswegen wegzunehmen ift, fo werden kaun wwey oder drey wirkliche Arten diefen Gefshlechte iibrig bleiben, and vielleicht genouler genommen, gar nur die erfte Art, der ARG. ArGo, und die sweyte, der A.VITREUS; wofern nicht ans allen jenen bisher bekannten, unter den $N_{a h m e n}$ von Abänderungen in diefe erfte Art zufammen gezogenen Stücken etwa etliche find, die folche beftändige, nicht zeränderliche, unter fich aber siemlich verfchiedene Eigenfchaften haben, dafs fie wwey oder drey befondere Arten ausmachen könnten.

Ceterum præcedentem definitionem noftram genericam ab omnibus quinque interim adoptatis fpeciebus abstraximus, ac fecundum earum proprietates compofuimus.

Omnes in eo conveniunt, quod ha. beant TESTAM Univalvem SPIRALEM b), vel involutam (A. Argo), vel CONVOLUTAM, cymbium feu naviculam ruditer æmulantem, qua ftructura cymbiformi feu naviculari Nautilis proprie dictis affimilant, lateribus vel leviter ftriatis ( $A$. Cornu, arcticus), vel

b) Id quod fub vocibus: SPIRALE, INVOLUTUM \& CONVOLUTUM, intelligamus, vid. in Nota a) ad Introduct. noftram in Genus NAUTILUS.

Unterdefjen haben wir vorftehende unfere Gefohlechts - Erklärung von allen funf aur $Z$ eit aufgenomimenen Arten ab. gesogen, und nach ihren Eigenfohaften verfufjet.

Alle Arten konmen darinn überein, days fie EINSCHALIG, SCHNIRKELoder SPIRALFöRMIG b), entweder EINGEROLLT (A. Argo), oder zUSAMMENGEROL L T find, und anf folche Art einen Nachen oder Schiffchen einigermasJen vorflellen, durch welchen nachenforrmigen oder fchiffartigen Bau fie den ei-

h) Was wir anier den Ausirücken: SPIRAL-oder SCHNIRTELFÖRMIG, EINGEROLLT OdeT IN SICH SELBST GEWUNDEN, nN ZUSAMMENGEROLLT CIEV ZUSAMAIENGEIVUN$D E N$ verfehen, findet man in der Annerk, a) wnferer Einleitung zum Gefoblecht SCHIFFEA, 
transverfim undulato - plicatis (A. Ar- gentlich Jogenannten Nautilis (unJern Sshifgo) \& interdum fimul granulatis (ejusd. fern) ähnlich werden; ihre Seiten find entVar. vulg. Reisbrey-Nautilus), compref- weder fanift geftreift (A. Cornu, arctifis, vel æqualibus (A. Argo, vitreus), vel cus) oder in die Quere wellenformig geinæqualibus, feil. altero latere plana- faltet (A. Argo) und bisweilen zugleich to, altero plus minusve convexo (A. Cor- gekörnt (eine Abanderung deffelben insgenu, arcticus). TESTA porro eft carinata, CARINa five Dorsum vel angufte, vel late retufum $\int$. canaliculatum, utrinque fubdentatum, dentibus mox oppofitis, mox alternis (A. Argo), vel per medium fubacutum dentatum (A. vitreus), vel convexiusculum $f$. obtufum, leviter ftriatum (A. Cornu, arcticus), fimulque transverfim fubnodulofo-coftatum (A. Cornu). Anfractus funt repente dilatati, excepto A. Arctico, vel obtecti (A. Argo), vel aperti, porro aut disjuncti ( $A$. Cornu), ant contigui, denique numero vel duo (A. Argo, vitreus), vel tres (A. Cornu), vel feptem apparenter tales, fed veri folum duo (A. arct.). Apertura vel fubcordato-oblonga (A. Argo), vel dilatatofubrotunda, margine fubreflexo repando (A, Cormu), vel oblongo-ovalis (A. vitreus), vel oblongo-coarctata (A. arcticus). mein Reisbrey - Nautilus), sufammengedriicht, entweder einander gleich (A. Argo, vitreus) oder ungleich, nehnlich eine Seite fach, die andere mehr oder weniger gewölbt (A. Cornu, arcticus). Die SCHALE ift ferner gekielt, der KIEL oder RücณEN bald eng, bald weit, eingedriìkt, oder rinnenförmig, auf beyden Seiten gesiihnt, wo die Zihne bald entgegen gefetst, bald abwechfelnd find, (A. Argo), oder iiber die Mitte etwas fcharf und geacihnselt (A. vitreus), oder ein wenig rundlich - erhaben, oder fumpf fein geftreift (A. Cormu, arct.), auch zugleich queriiber ein wenig erhaben geribbt (A.Cornu). Die WI N D U N G EN nehmen fichell $\approx u$ und find erveitert, ausgenommen der $\mathrm{A}$. Argo, entweder bedeckt (A. Argo), oder. gans fichtbar, ferner abfehend (A. Cornu) oder anliegend, endlich der Zahl nach bald zwey (A. Argo, vitreus) bald drey, (A. Cornu) oder dem Anfshein nach fieben, wirklich aber nur wwey (A. arcticus. Die Miindung ift entweder $f a f t$ her förmig länglich (A. Argo), oder ansgebreitet rundlich, mit etwas ungefolnagenem ansgefinveiftem Rande (A. Cormu), oder langlich oual (A. vitreus), oder länglich verengert (A. arcticus). 
Breviter adhuc notamus, epitheton:

Kiirslich bemerken wir noch, dafs das MEMBRANACEA, quod Limnus in de- Beywort MEMBRANACEA (HAUTARfinitione fur generica adpofuit, propter- TIG DüNN), das Linne in feiner Geea, quod non folum hujus generis, fed fohleshts-Erklänng hinsugefetsthat, defso adhuc permultis aliorum generum teftis wegen, weil diefe Eigenfthaft nicht bloss commune eft, fupervacaneum \& huc non pertinens vifum fudio a nobis omirum efle.

Color plurimum albus eft in Arg. Argo, interdum partim albidus flavefcente vel fufco mixtus.

Patria f. locus, ubi inveniuntur: Oceanus, mare Indize orientalis, mediterraneum (A. Argo); Oceanus ad Caput bonæ Spei Afric. Atlanticus ad Portugall. (A.Cornu); mare ad Gronlandiam (A. arct.).

Mensura: A. Argo 5 unc. - 1 ped. long. A. vitrei $2 \frac{1}{2}-3$ unc.long. A. Cornu $4-5$ lin. long.; A. arct. $3 \frac{1}{2}$ lin. diam.

De his quatuor fpeciebus nomnifi unicam fcopo noftro infervientem, Argonautam Cornu, habemus, quam hic fifimus. diefen Gefchlechte eigen, fondern noch vielen von andern Gefichlechtern gennein ift, uns iiberfiilfig und hieher nicht gehörig geftiienen hat, und wir alfo folches vor. Setalich weggelaffen haben. Die

FARBE ift meiftens weifs, bey dem A. Argo bisweilen weifslivh mit etwas gelb oder braunlich genifsit. Das

VATERLAND oder der Ort, wo je gefunden werden, ift dis Weltnuer, das oftindifche, mitiellindifche (A. Argo), das beym Vorgebirge der guten Hoffuning in Afrika, atlantifche bey Portugall (A. Cornu), bey Grönland (A. Arcticus).

Grösse vom Argo 5 Zoll bis I Schuh in der Länge; A. vitreus $2 \frac{1}{2}-3$ Zoll lang; A. Cornu 4-5 Lin. lang; A. arct. $3 \frac{x}{2}$ Lin, in Durchineffer.

$V$ on diefen 4 Arten haben wir num eine Art, die su unferm Zwecke dienet, den Arg. Connu, den wir hier darlegen. 


\section{ARGONAUTA CORNU \\ WIDDERHORN ARGONAUTE.}

Germ, das antike Trinkhorn, Spengler.

Der kleine Papier-Nautilus, Chennizz.

Der Papier-Natilus mit fichtbaren Wirdungen, Schrite,

$$
\text { T A B. I. Fig. a. b. c. d. c. }
$$

Gall. le Cornet de Chafreur, Favanu.

Linn. Syft. nat. cdit. XIII. Gmel. p. 3363. $\int_{p} .4$.

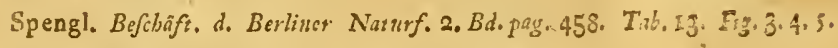

Favann. Conclyl. p. 599 \& 665. Tab. 5. Fig. I.

Schröt. Coulch. Ketut, 1. Bd.p. 6. Nro. 2.

Chemnitz Conch. Cab. 10. Bd. p. 4. Tab. 137. Fig. 1271.-1373.

Schreibers Concb. Kenmti, I. Bd, p. 2. t. $_{4}$.

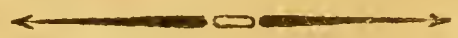

TESTA fubrotundata fpiralis convoluta, tenuis, fubpellucida, anfractibus tribus (in modum cornu arietis inflexis) con. fpicuis f. apertis, disjunctis, amplitudine repente decrefcentibus; fpira tota horizontaliter planata, ftriis arcuatis fubradiantibus levifimis notata (Fig. b.); lalere oppofito $f$. inferiore elevatiore medio retufo f. quafi umbilicato, fimiliter firiato, (Fig. c.); dorfo convexo, utrinque angulato, infra obtufiore, transverfim f. ductui anfractuum parallele coftato, coftis quinque obtufis interrupte leriter nodulofis, fulcis quatuor intermediis planis radiatim fubtiliter, fed denfe firia-
Die SCHALE ift etwis randizith, fpiral- oder fomirkalformig anfanmen gerollt, dülnn, ein weitig durchfichtig, hat drey fichtbare zon einander abfehende (nach Art eincs Widderhorus eingebogene) Windungen, die in der Dicke jühe abnelinen ; der Wirbel rogt gar nicht hervor, fondern ift horisontal gleich unul völlig fach, mit logenförmigen siemlicis frahlartig anslarfenden feinen Streifsi: gezeichnet (Fig. b.). Die entgegen gefet $\approx$ te oder untere Seite ift melr crhaten, in der Mitte eingedrückt, oder gleichjant genabelt, und auf gleiche Wreife gejireift (Fig. c.). Der kücken ift rund erhater, 
tis(Fig.d, e.); apertura magna dilatata auf beyden Seiten woinkelig, doch unten fubrotunda, margine fubreflexo, repando. fumpfer, nach der Schale, d. i. mit dem Lauf der Windungen parallel liegen finf glatte, Anmpfe, abjat:weife Schuachkno. tige Ribben, welche avifichen fich vier fache Furchen, oder vertiefte Zwifchenrâu. me bilden, die gegen den Mittelpunkt $\approx u$ fralig, wart, aber dicht geftreift find, (Fig. d. e.). Die Mündung ift großs, er. weitert, beynahe rund, mit einen etwas ausgebogenen und ousgefihweiften Rande.

COLOR, albus.

PATRIA, Oceanus ad Promontorium bonæ Spei, Spengl.; mare ind. orient. Favdizn. ; atlanticum ad litt. Portugalliae, schreibers.

MENSURA, 4 lin. diam. major. $2 \frac{4}{5}$ lin. diam, min. $2 \frac{2}{5}$ l. altitud. max. $1 \frac{\pi}{3}$ 1. alt. $\mathrm{min}$.

Not. Niff celeberrimorum in conchyliologia peritifimorum virorum D. D. Spengleri, Schröteri \& Chemnitaii contra nos ftaret auctoritas, qui cochleam hanc generi Linnæano Argonauta dicto appropriarunt, magnopere inclinaremus eam ad Turbines \& quidem juxta Delphinum L. transferre, quibuscum, fpinofum ramofumque fi demas, magnam habet affinitatem, etiam quoad orificiım, quod in noftro fpecimine non quadrangulare, uti vulgo defcribitur, fed rotundatum apparet.
$F A R B E$, weißs.

$V A T E R L A N D$, das Weltmeer an Vorgebirge der guten Hoffnung, Spengler; oftindifche Meer, Favann; das at. lantifche an der Kiifte von Portugall, Schreibers.

GRÖSSE, 4 Linien groß. Durchn.

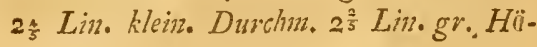

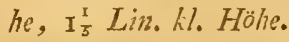

Anmerk. Wenn wir nicht das Anjehen erfahrner Konchyliologen, eines Spenglers, Schröters und Chemnitz widev uns hatten, die diefe Schnecke in das Linn. Gefchlecht Argonauta gefetst haben, fo waren wir Sehr geneigt, diefolbe $z u$ den Mondfchnecken neben den Delphin (Turbo Delphinus L.) zu fet:ein, nit welchenz fie, das Domige und Lappige oder Aefti. ge ausgenommen, fehr viel gemein hat, auch in Rïckficht der Miindung, die an unferm Stiiske niicht vieveckig, wie fie insgemein befohrieben worden ift, fondern rundich fich zeigt. 


\section{NAUTILUS.}

SCHIFFER。

IESTA univalvis, fpiralis involuta, poly- Die SCHALE befthet ans einem Sticke, ift thalamia; fchnirkelfömig eingerollt, vielkanmerig; die

ANFRACTUS contigui, obtecti, concame. rati;

THALAMI fimplices, rarius iterum Iocu. lati, transverfim diflincti

ISTHMIS feu Difepimentis fornicatis perforatis

SIPHONE quandoqne teftaceo interrupto, faepe contiguo, includente nervum, vel tubulun cutaccuns per totam teltam continuum;

ORIFICIUM vel fimplex, vel pluribus fo. raminibus compofitum.

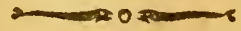

Cum femper ægre \& folo veritatis amo. re, neutiquam novationis pruritu, ducti aliam, quam quæ a claris Viris ftabilita $\&$ unanimi fere confenfu recepta eft, fequamur fententiam, fic etiam hac vice in conftituendo \& arctioribus limitibus, quam ab Illuftri Linnæo factum eft, coërcendo Nautili genere non nifi urgente, uti faltem nobis perfuafum habemus, necellitate commoti fumus, ut ea teftacea uniralvia polythalamia, quæ licet ftructura habituque magna ex parte lon-
WIND UNGEN, fut anliegend, bedeckt, in meb. rere Ficher eingetbeilt; die

KAMMERN fund geroölynlich einfach, feltner wie. dernum in kleincre Ficher eingetbeilt, it die Quere durch

SCHEIDEẄ̈ANDE unterfchiedcn, die gemölbt sund durcblöcbert find mittelf einer

SAUGRÖHRE, die ans fchaligen Beftandtbeiler befelht, die mauchosabl in einem fortgehee sund eine durch die ganze Scbale fortlanfende häutige Röhre einfchlinfst; die

MÜNDUNG ift bald einfach, bald beffelt fic aus msbrem öfiningen.

$D_{a}$.wir alleweit fehwer daran kommen and keineswegs dur h Neutrungsfucht geleitet werden, eine andere Meining aufsuftellen, als diejenige, die von beriihmten Mannern feftgefetat and durh einhellige Übereinftimmung aufgenommen ift, fo wurden wir auch jetst bey genauer Beftimmung des Gefshlechts Nautilus und deffen Einfchranknug in engere Grinzen, als das ron Limné fo genannte hat, bioß durch die dringende Nothwendigkeit, wie wir wemigfteras uizeres Theils dufïr bala 
ge inter fe diverfa in unum ideoque ni- ten, dahin veranlafet, daß wir diejenimis laxum genus a Linnæo coacervata $\&$ quali coarctata funt, in quatuor dispertiverimus genera, refervata primo ge. neri denominatione $N$ autili, reliquis Hammonii, Litui \& Orthoceratis nomine im. pofito, quæ quidem non inconfueta, fed olim jam quibusclam fpeciebus data funt. Conatus noftros dijudicent doctioris ingenii neutriusque partis ftudiof Conchylioloei.

Ut character, guo hae quatuor genera a fe invicem differunt, clarius elucefcat citusque pollit concipi, cujuslibet trium polteriorum definitiones hic fi. fiimus.

HAMMONIUM tefia uniralvi fpira1i, convoluta, polythalamia ; Anfractibus vel utrinque, vel folum fupra confpicuis, contiguis, concameratis; Thalamis fimplicibus, vel rarifime iterum concameratis; Ifthmis transverfis fornicatis pertufis Siphonze teftaceo laterali (in plu. ribus, an in omnibus?); Orificio lineari in latere inferiore, rarius antice.

gen Schalthieve, deven Gohaule nur aus einem Houptfliicke befteht, aber vielfächerig ift, zoelche insgefanunt, fo fehr verfchicden fie auch grolfen Theils ihren Bou und äuferlichen Anfehen nach von einander fand, Limne in ein einsiges und $d a-$ her all:ufehr ausgedehntes Gefchlecht \$ufommengehäuft, und gleichfan anfannmeaz gedränget hat, in vier Gefihlechter sertheilten fuir das erfte den Nohnen Nautilus beybehielten, den drey übrigen aber die Benennung Hammonium, Lituus und Orthoceras beylegten, die awm an ficl aicht neu, fondern ehemals fehon verfchiedenen Avten gegeben worden find. Ob wir hievin wohl gethan haben, iiberlaffen wir Konchyliologen von tieferer Einficht und unbefongenen Gemithe anv Bentheilung.

Danit aber der charaliteriftifche Untevfohied wwifhen diefen vier Gof.hlechtern beffer erhelle und gefohwinder eingefehera werden möge, wollen wir auch die Erklärungen der drey folgenden Gef:hleclite gleich hierher fotzen.

Das HAMMONIUM ift einfchalig, felmirkelformig, aufannnen gerollt, und vielkannzerig; die Windungen fand entweder auf beyden Seiten, oder wenigftens oben fichtbar, anliegend, gekanninert; die Kammern einfach, oder fehr felten wiedevun mit mehrern Fichern verfehen, in die Quere durch Scheidewände unterfchieden, die gewölbt und durchlöchert fnnd von einer hartfchaligen Saugröhre, 
LITUUS tefta univalvi recta vel fubarcuata, apice incurvato-fpirali, polythalamia; Anfractibus rel contiguis, vel disjunctis, mox confpicuis, mox obtectis; Thalamis fimplicibus transverfe difinctis Ifthmis fornicatis; Orificio vel centrali orbiculari, vel transverfim lineari interrupto, aut fimplici aut duplici ferie pororum (an fimul apertura fipho. nis?).

ORTHOCERAS tefta univalvi porrecta, vel ftricta, vel fubarcuata, apice recto, quandoque inflexo, polythalamia; Thalamis fimplicibus, aut uniferiatis, aut alternantibus; Diffepinentis vel transverfis, vel obliquis, fornicatis, vel angulato-flexuofis; Siphone (in quibusdam tefiaceo, an in omnibus?) vel centrali, vel laterali ; Orificio orbiculari, vel ovali, vel lineari, aut centrali, aut laterali (an fimul fiphonis uItima apertura?). die (bey den meiften, ob bey allen?) ans ciner Seite angebracht ift; die Mündung ift frichförmig, an der untern Seite, felten vorwärts.

Der LITUUS ift einfshalig, gerade, manchmahl etwas gebogen, in beyden Fillen an obern Theile oder an der Spitse fibnirkelförmig eingekrümnt, vielkanmerig; aie Windungen der Spitze fund ent:veder anliegend, oder abfehend, bald fichtbar, bald bedelit ; die Kammern einfach, nach der Quere abgetheilt durch gewölbte Scheidewände; die Mündung ift bald in der Mitte kreisrund, oder queriiber ftrichförnig unterbrochen, bald in einfacher, bald in doppelter Reihe von kleinen Löchern (ift vielleicht auch $\approx u$ gleich die Oeffunng der Saugröhre?).

Das ORTHOCERAS ift einfchalig, ansgeftreckt, entweder ganz gerade, oder etwas gekriunnnt, an der Spitze gewöhn. lich gana gerade, anveilen ein wenig umgebogen, vielkannerig; die Kammern find einfach, entweder in gevader Reihe, oder wechfelweife uber einander ftehend; die Scheidewände ftehen entweder quer iiber oder fchief, find entweder gewölbt, oder winkelig bin und her gebo. gen; die (in einigen Arten, vielleicht in allen, havtfchalige) Saugröhre ift bald in der Mitte, bald an der Seite; die Mündung entweder kreisrund, oder oval, oder. frichförmig, bald in der Mitte, bald an der Seite (etwa augleich die letzte Oeffnung der Saugröhre?'). 
Ex his quatuor juxta fe politis definitionibus genericis, defumtis potifimum a fpeciminibus naturalibus, adhibita par. ciori comparatione cum quibusdam folfilibus (licet attentiorem horum contem. plationem ad majorem illorum illuftrationem non parum nobis contulife candide fateamur), fatis patebit, qualis intercedat differentia.

Differt nimirum NAUTILUS anfracti. bus obtectis ab Hammonio. Porro differt forma Jpirali ab Orthocerate, \& denique forma tota pirali a Lituo.

HAMMONIUM difinguitur anfractibus omnibus vel utrinque vel faltem altero latere confpicuis a Nautilo; frructura Spirali ab Orthocerate, \& tota Jpirrali a Lituo.

LITUUS differt parte porrecta a Nautilo \& Hammonio; et parte fpirali ab Orthocerate.

ORTHOCERAS denique difcedit forma tota fricta, vel plus minusve arcua$t a$, a tribus præcedentibus generibus, licet inter hac duo pofteriora genera, $\mathrm{Li}$ tuum nempe \& Orthoceras, ficut inter tho anteriora, Nautilum \& Eismmonium,
Diefe neben einander gefetate vier Gefchlechts-Erklärungen find hauptfachlich nach Stücken in ihrcm natiurlichen $Z$ ilfande entworfen. Doch hat man auchpar. fonn Vergleichnngen mit einigen fo:flen angeftellet; dabey wir freyniiithig geftehen, daßs die aufnerkfonneve Betrachtung diefer letatern uns nicht wenig all nähe. ver Beleuchtung jener, der natiorlichen, behiilfich gewefen ift. Aus diefen Erkla. rungen nun wird zur. Genïge der Unterfohied erhellen, der awifhen un'ern vier Gefchlechtern obwaltet.

Der NAUTIL US unterfcheidet fich nchmlich durch die bedeckten Windungen von Hanmoniun, ferner dirch die fchnirkelartige Geftult vonu Orthoceras, und erdlich durch den vollkommen fchnirkel. förmigen Baru von Lituus.

Dirs H.1MMONIUM seichet fici durch alle feine entreder beyderfeits, oder wenigftens einerye its fichtbare Windungen vom Nautilus aus, durch den fchnirkelförmigen Bau rom Orthoceras, und durch den ganz fchnirkelförmigen vom Lituns.

Der LITUUS weicht durch den gerade ausgeftreckten Theil rom $N_{\text {autilus }}$ und Hannioniun ab, wnd durch den fpiralförmigen Theil vom Orthoceras.

Das ORTHOCERAS endlich snzerScheidet fich durch feine völlig gerade, oder doch nur melir oder mindir gekrümmte Geftalt von den drey vorgehenden Geforlechtern, obwohl nicht kann gelangnetwerder, dirs arijehen den beyden letatern 
magnam verfari affinitaten negari haud q̨uaquam pollit.

Ceterum haec quatuor genera diffesunt ab omuibus aliis hucusque notis tefaceorum generibus in eo, quod polythalamia fint, reliqua vero omnia folummodo fimplici caritate gaudeant, li ali. quot ferpulas excipias, (Limæus de unica folum fpecic locutus eft, quam ferpu-

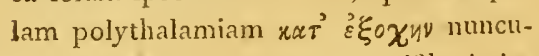
pavit, cum tamen alias etiam ilthmis interfeptas invenerimus), quæ autem viciffim a.noftris generibus in eo difcedunt, quod adhereant aliis corporibus, quod fecus in prafatis generibus, qua libera iunt, quo ron folum cum ferpula feminulo Linn. (quæ vero nobis proprium genus Frumentarii nomine confituit), fed \& hoc conveniumt, quod dicta Serp. Seminulum qnoque multilocularis fit.

Ad Polythalamia referri poffunt Tefre hammoniformes Dui. Abbatis Soldani Teftaceographia T. I. P. I. tab. 68-8.3. \& certo refpectn variæ polymorphæ P. II. tab. 109 - finem difperfe.

Circa genus nofirum Nantilus dictum, cui, nomen hoc jam tritum, a Nautilo Ponpilio, qui agmen ducit, depromptum, \& a Limieo generi fito impofitum, refervare maluimus, quam norum dem Lituus nehnlich und Orthoceras, fo wie awifchen den beyden erftem, dem Natrtilus und Hạmonium, fehr viel Übereinflimanung herrfche.

Übrigens winterficheiden fich diefe vie? Gefchlechter von allen ïbrigen bisher bekamnten Schalthier-Gefchiechtern dadurch, $d a / s$ fe vielkammerig find, alle übrige aber mur eine cinfache Höhlung haben, etliche Serpulce ansgenominen (Linne hat nur eine einsige Art genannt, die er vorzugsweife die vielkammerige nannte, $d a$ wir doch auch andere mit Scheidewiindens begabte gefunden habenl), welche aber wiederum darin zon unfern Gejihlechtern abgehen, daßs fie ml, andere Körper fici anfetzen, da hingegen ervähnte Gefchlechter frey find, worin fie nicht num wit der Serpula Seminnlun Linn. (die aber bey uns ein eigenes Gefotitecht wnter dent Nahmen Frumentarium ausnachit), fondern auch darin ilbereinkomnuen, daßs gedachte Serpula Semin. auch mehrfacherig ift. $Z u$ den vielkannmerigen Konchylies könuen auch gereshnet werden die 7 efte himmoniformes des Hrn. Abts Soldani in feiner Teftaceographia T. I. P.I. Tal. 68--83, und gewiffermaffen anch verfchiedene vielerley geftaltete, die fich im P. II. Tab. 109 bis ans Ende zerffreut finden.

Fn Aifehung unferes Gefshlechtes, Nautilus genamut, fiir welches wir diefen bereits lekannten vom Nautilus Pompilius L. als der erflen Art hergenommenen und von Linné Seinem Gefinlechte beygelegten 
magis ignotum conclere, quædam adhuc monenda funt; præfertim quæ ad fpecialiorem partium ftructuram variarum fpecierum fpectant, \& ad faciliorem harum cognitionem contribuere pollunt.

a. Omues hujus generis fpecies habent TESTAM vel orbicularem $f$, rotundatam, rel fubovalem, vel fubovatam, aut anbitu integro, aut repando, aut angulato, aut obtufo, aut carinato, mox dentato, mox fpiriofo feu aculeato, mox inermi, fuperficie lavi, ftriata, firigillata, coftata, punctata, punctis vel elevatis, vel excavatis, granulata, tuberculata, tuberculis vel globu'aribus, vel irregularibus. Porro tella eft inzoluta, ubi nimirum anfractus interiores fpiraliter circumvolvuntur ab exterioribus ita, ut utroque latere nomifi extimus confpicuus fit, (anfractibus obtectis), ex. gr. Nautilus Vortex gyris partim apertis (Tab. 2. fig. 7.) \& Naut. lenticularis per axem dimidiatus (Tab. 7. fig. d.) Hac ftructura Nautilus conrenit cum Cypræis \& aliquot Bullis Linnæi, cum contra ille difcrepet ab omnibus reliquis teftaceis fpiraliter contortis, quibus plurimis eft tefta convoluta, fcilicet ubi anfractus interiores ab exterioribus ita fpiraliter circumvolvuntur, ut omnes vel utrinque, vel faltem altero latere manifefte appareant, (anfractibus apertis five confpicuis). Hujusmodi funt: Argonautæ, Hammonia, Co.
Nahmen lieber haben beybehaiter, als ei. nen nenen weniger bekannten finöpfen wollen, komnt nosh ein und anderes $\approx u$ bemerken, befonders was den waiheren Bau aes. Theile an verfchiedenen Arten angelot, und an leichterer henntnißs derfelben beytragen kann.

a. Alle Arten diefes Gefihlechts haben entweder eine kreisrunde, oder etwas ovale, oder sienulich eyförmige SCHALE, deren Umkreis entweder gans, oder ausgefhweift, oder winkelig, oder ftumpf, oder gekielt, bald gesahnt, bald dornig, oder ftachelig, bald ohue dergleichen Auswiichle ift, auf der Oberfläche glatt, geftreift, geftrichelt, geribbt, erhaben oder vertieft punktirt, gekörnt, höckerig, mit K"ugelchen oder unregelmajßigen Höckerchen. Ferner ift die Schale eingerollt, oder in fich felbft gewunden, wo nehmlich die innern Windungen von den äusern fchnirkelförnig umwunden, oder fo cingewickelt werden, dafs auf jeder Seite blofs die änserfte fichtlar ift (revdeikte Windungen), $\approx B$. der Nantilus Vortex nit $\approx$ um Theil geöffneten Windungen (Tab. 2. fig. h), ingleichen $N$. lenticularis durch die Axe sur Hailfte getheilet (7ab. 7. ig. d.). Durch diefen Bau kommt der Schiffer mit den Porzellanfchnecken (Cyprea L.) und einigen Blafenfchnecken (Bulla L.) iiberein; hingegen unterfuseidet er fich von allen andern Schizirkelförnig gewundenen Schalthieren, die eine zufammen gewundene oder zufammen gerollte Schale haben, nehmlich, 
ni, quædam Bulre \& Volutæ, omniaque illa tefiacea, qux fenfu Linnæi fpira regulari gaudent, quibus accenfeantur etiam aliquot Serpulæ ").

a) Vocibns involutum \& convolutum in aliquali alio fenfu utimur, \& ni fallimur, difinctiori, quam in Murray fundam. tefaceolog. (Linn.) explicate funt. Sic etiam tefta fpiralis nobjs ef, ubi paries teftx externus continuus circa centrum vel axin ita volvitur, ut vertendo fpatium a centro vel ab axi antrorfum proportionate (vel lente, vel sepente) dilatetur.

Antrorfum, quod a centro vel axe verfus la. tiorem partem \& aperturam $f$. in polythalamiis verfus ultimum thalamum vergit,

Retrorfum, quod a parte latiori verfus angufiorem leu centrum five primum thalamum ver. sit. E. g. Ifthmi (in polythalaniis) antrorfum convexi (f. retrorfum concegi) (unt, qui latus con* vexum verfus partem tefte dilatatiorem ( $f . \mathrm{la}^{-}$ tus concavum verfus anguftiorem) vertunt. $1 \mathrm{ft} \mathrm{bmi}$ retrorfum convexi (โ. antrorfum concavis), qui latus convexum verfus partem anguftiorem (โ. lasus concayum verfus laxiorem partem) vertunt. wo die innern Windungen von den äu/sern dergeftalt finnirkelformig unwounden werden, dass alle entrveder anf beyden Seiten, oder wenigfens anf einer Seite von einonder unterfchieden werden können (fichtbare Windungen), dergleichen find die Argonauten, Anonshörner (Hummonia), Kegelfilinecken, einige B'afenf:hnecken, die Walzen- oder Faltenf chnecken, und alle jene Schalthiere, die na:h der Linneifchen Bedeutung regelmäfsige Windungen haber, welshen aush einige Serpulce beyzusaihlen find a).

a) Wir gebrauchen die Würes: : involutum (eingerollt) und convolutum (zu[ammengerollt) in einer etwas anderen, und wenn wir nielt irrest. dextlichern Redestung, als fie in Muryay's Fundawsentis tefiaceologic (linnean.) erklürs find; fo nennen wir aneb diejenige Schabe fchnirkelförunig gewunten, wo die äfsere aus eizien Stücke tefieliende IVand $n \mathrm{~m}$ den Mittelpunkt oder $\mathrm{xm}$ die Axe fo gewunden ifi, dafs beym U'w wilzes der Raum vom Mittelpunkt oiler von der Axe aus vorwärts verlältnifsmilf,ig entweder allmäblig, oller fcbrell, fich erweitert.

Vorwärts beiffen wiv, was fich vom Mittelfunkt oder der Axe aus geyen den weitern $\mathrm{T}$ bei und die Öfrung, oder ley den vielkummerigen gegen die letzte Kam-ner $z$ u wendet.

Rückwärts ifi, was vnm weitern Theile gegen den engern oder gegen den Mittelpunkt, oder die erfe (iunerfte) Kanner $=$ u fich kebret. $Z, B$. Die vorwärts geworlbten Schei.ewriode (heyden vielkapmerigen) oder rûckwärts hohlen find duejenigen, weiche die gewölbte cider enkene Seite gegen den rweiter:en abeil der scibale, oderdie

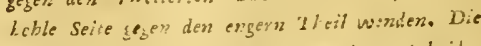
rückwärts curivexen o'er gexölbten sckeidenü̈le, oder veswärts tho len, fint, welche ib-

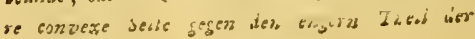


b. ANFRACTUS funt omnes conti. tigui, extus mox laves, mox friati, mox coltati, vel punctati, punctis elevatis, aut excavatis, vel granulati, vel tuberculati, aut planati, aut torulof b) feu articulati, intus concamerati, five in plura loculamenta feu thalamos divifi.

Antroysum et retrosfum ergo non fignificat id, quod anticsm et pofficsm, quibus vocibus Linnzus in defcriptione Labri fignificatione fpecialiori utitur; purs antica enim labri ipfr eft, qua ad Ppirain vergit, et pofica, squre ad caudam,

b) Torofum, torulosum in fenfu Linnei idem eft, ac tumidum, valde convexum. Articuli torofi, i, e. valde collvexi, e, gr. Naut. (nobis Orthoceras) Raphaniftrum, Kaphanus, Granum, Radicula Linn.; hinc Anfractus torulofi : ii anfractus, qui inter geuicula tumidiores funt (Fundam, tefia. ceolog. in Schröteri Ephemerid, litter, hift. nat. (germ, Für die Litteratur d. Naturg, 1-82. Tom. I. p. 214. (Naut, Beccarii L) five anfractus valde convexo-articulati, id eft, qui aliquot geniculis [. coarctationibus in aliquot conve. xos articulos difincti funt. Non tortus/s (gekrümrat, gedreht), uti Dno. Schsötero (Einleit. Conch, Kenntn. T. I. p. 11, ad N. Becc.) vifum et a D. Grielino (Linn. Tyft, nat, ed, XIII. T, 6. p. 3370, Sp. 4.) repetitum eff. b. Die WINDUNGEN find alle anliegend, aussen bald glatt, bald geftreift, bald gevilut, entweder punktirt mit erhalonen oder vertieften Punkten, oder geliönt, oder höckerig, entweder niedergedriickt oder fach, oder rund crhaben b), oder gegliedert, inwendig gekanmert oder in viele Fïcher oder Kanntern eingetheilet.

C 2

Scbale, oder ibre boble seitc gegen den weitern Theil hinkelren.

Vorwärts nud rüekwärts bedextet demnach nicht das, was anticum and pofticum, welcher wörter fich Linne in Befchreibung der änfsern Schalenlifpe in befonderem Sinze bediente, anzigen foll; denn pars antica, der vordere Theil des Lipfe, leifrt beyibm derjenige, der fich nach der Sfitze zus'endet, rul poffica, der hintere Theil, derjenige, der ficb gegen den Schwanz, (die wntere Verläargerung der Scliale) lin neiget.

b) Torofurn, torulofum ift im linneifchen Sinn foriel als anfgeblafen, ftark erbaben, boch sewölbt; Articuli torofi finl alfo ziemlich sund erbabene oder gewilbte Glietler, ₹. B. Nast. (robis Ortboceras) Ra.baniftrum, Rapbanus, Granum, Radicula Lim, folglicb anfractus torulofi erbaben gegliederte Windrugen, anfractus, qui inter gericula tumidiores font (Funiam. teftaced. in Schröters Tegbuch. Für die Litteratur der Naturg. 1782, I. Bd. S.214) d.i. die aus gleichen gewöluten Gliedern zufammengefetzte U'indungen, oder dife durch mekrere Einzichungen (Verengeoungen, gericula) in etliche gewölb te (runtl erbabene, contexe) Glieder absesbeilt find (z. B. Nast. Feccurii Linn. oder unfer Hummonisu Becearism). Nicht etwa torluofi, gekrimmte, gedielie Windungen, wie es Herrn Schröter (in Seiner Eivleitung in die Conchylienkenntn. 1. Bd. S. 11. beym N. Beccarii) gefcbienen bat und von Hin. Gmelin (Linn. Syft, N. edit, XIII, T, 6. p. 3370, Sp. 4.) wiederbolet worden ijt. 
c. THALAMI vel fimplices $f$. uniloculares funt (tefta fimpliciter concamerata), vel denuo in multos loculos transverfim fubdiftinctis (tefta duplicato - concamerata). Thalami etiam differunt numero, in extimo anfractu plurimarum fpecierum vifuntur $12-14$, quandoque 5 (N. coftatus), $5-9$ (N. Calcar), rarius $20,24,30,48$, (præertim in Naut, duplicato-concameratis, (Naut. crispus, angulatus, craticulatus, ftrigilatus ,), 52 (N. aduncus). In paucis fpeciebus nulli thalani extrinfecus confpicui funt (N. Mamilla, Orbiculus, Spengleri). Refpectu curvitatis thalamorum quoque magna eft diverfitas, quædam enim fpecies, præcipue duplicato-concameratæ, thalamos habent valde arcuatos, quorum aliquot duas tertias ambitus occupant, aliæ contra tam leviter curvatos, ut iftumi fere recti appareant. Denique latitudo eorum etiam variat, atque in duplicato-concameratis ifthmorum trans. verfalium parvorum $\mathrm{f}$. fecundariorum loculorum diftantia. Propter magnum hoc evidens inter thalamos tam quoad fructuram, quam numerum, intercedens discrimen, cum nimirum fpecimina obveniant, quibus funt thalami, ut ita dicamus, primarii, circa $5^{\circ}-5^{2}$ denuo in locu!os fecundarios circiter 1000 (Naut. aduncus) ifthmulis transverfis, feu ambitui teftæ parallelis, rectis, non arcuatis, uti primarii, fubdivifi, nobis genus boc noftrum in duas familias, in limpli- c. Die KAMMERN find bald einfact, bald einficherig (einfachkanmerige Schale) bald abernahl in viele kleinere Ficher in die Queve nnterabgetheilt, (doppeltkammerige Sihale). Die Krmmern find auch in der Ansahl unter fivh verfohieden, indem man in der ciußserften Windung der mehreften Arten 12 - 14 fehen kann, manchmal 5 (N. coftatus), 5 - 9. ( $N$. Caliar) feltner $20-24,30,48$, vorwiiglich an den doppeltgekanmerten (ans $N$ crispus, angulatus, craticulatus, frigilatus), 52 an $\mathrm{N}$. adunsus. Wenige Arten find, wo aufien gar keine Kammern fichtbar find ( $N$. Mammilla, Orbiculus, Spengleri). In Anfehung der Krilmnung der Kanmern herr.joht ebenfalls eine groSse Verfchiedenheit; inden einige Arten, befonders die doppelt kanmerigen, ftark gebogene Kammern haben, wovon einige zwey Drittheile des Unnkreifes ansmachen, andere hingegen fo wenig gebogene, dafs die Scheidewainde faft gerade evfoheinen. Endicls ift auch ihre Breite ungleich, und in den doppeltkammerigen die Entfermung der in die Quere flehenden Scheidewinde der kieinen Fäher von einander, bald gröfser, bald kleiner. Wegen diefes auffallenden Unterfchieds der Kammern foivohl in Anfehing des Banes, als der Anzahl, inden mehnlich Stücke vorkonnen, die ungefilhr $5^{\circ}-52$ Hauptkummern, wemn man fie fo nennen will, haben, die wiedernm in beyläufig 1000 kleine oder, fo au fagen, Niebenkanmern 
citer nempe et duplicato - concameratas difpefcentibus vitio verfurus erit facile nemo. Utrum vero hujus pofterioris familiæ fpecies a hocce genere potius feparandæe inque proprium genus coërcendx effent, porro an Siphone \& hoc cafu cujus fructuræ, item quali orificio gaudeant, denique an incola hujusmodí teftarum iis, qui teftas aliarum fpecierum primæ familiæ inhabitaut, aliquo modo fimilis fit, vel fi cum co teftæ ftructuram comparamus, forte ab iis diverfam habeat formam (licet de incolis hic fermo uon eft, cum nomnifi domunculas eorum tractemus), propter fpecierum inopiam fpecininumque magnam raritatem, atque prædictarum partium necelario præfumendam minutiem, dijudicare non polfumus.

\section{d. DISSEPIMENTA, ISTHMI,} PARIEETES INTERGERINI, INTERSEPTA, quibus anfractuum fpatium vacuum in plura loculamenta, thalamos, cameras, cellulas transverfim, vel potius a centro radiation proportionate an.
(Nout. aduncus) durch quer ftehende oder mit dem Unkreis der Schale parallele gerade Scheidewinnde (nicht gebogene, wie die Hauptfcheidewände) unterabgetheilt fand, haben wir diefes nneer. Gefchlecht in zwey Familien, nelmlich in folche mit einfachen Kammern, und in doppelt gekammerte, eingetheilt, welches uns wohl fo leicht Niemand verdenken wird. Ob aler die Aiten diefer letstern Fanilie von diefen Gefohlecht lieber gans abaufondern, und ein eigenes Gefohlecht daraus an errichten wäre; ferner of fie einen Sipho, und wenn dicss wire, von welchem Bau, ingleichen was fie fuir eine Mündung haben; endivh ob der Bewolner dergleichen Schrlen denen, die in den Schaltn anderer Arten ans der erftern Fanilie wohnen, einigernuafsen ähnlich fey, oder wenn man den Bau der Silale mit inn anfannuenhilt, vielleivit eine von ihmen unterfchiedene Geftalt habe, (olwohl von den Einwohnern hier die Re'e nicht yft, da wir bloss von ihren Gehiufsch hondein), hönnen wir aus Mongel genuglaner Arten und wegen grofser Seltenheit der Sticke, aush in Ai.jhung der nothwendig au zermuthenden Kleinheit gedachter Theile, darriber heine Entf-heidung fillen.

d. Die SCHEIDEW INDE, ZWISCHENWW IONDE, mittelf welcher der leeve Raum der Windungen in nuehrere Facher, Kanmern oder Zellen, der Quere nach, oder vielnehr von Mittelpunht ans frohlig vowworts in dor Weite verhailtnifsniissig 
trorfum amplitudine crefcentia dividitur, furt fornicata feu convexo - concava, vel perfecte, vel quandoque fubflexuola.

Notatu dignum, omnes, quas habemus vidimusque lunjus generis fpecies microfcopicas, diffepimentis antrorfum convexis præditas, neque quidem duplicato-cencameratas exceptas efre, \& nonnifi Nautilum Pompilium onmium inter naturales congeneres magnitudine longe principem folum ifthmis antrorfum concavis gaudere. Diximns inter naturales; inter folliles enim dantur, licet rarius, Nautilitæ longe majoris molis, ut \& minores, ifthmis antrorfum concavis gaudentes, fed non inter microfcopicos, fi fpecimen a Dno. Abbate Soldari, cujus iconibus faepius, fed uon femper fides habenda, in tentamine fuo oryctographico, italico idiomate edito ${ }^{c}$ ) tab. 2. fig. 18. A. rite reprofentatum eft, hoc ex omnibus ipfius iconibus, atque mul. tis millibus nautilorum minutorum, quos polidemus \& vidimus, unicum exftat, quod diffepimentis fuis antrorfum concavis a ceteris $f_{p}$ eciebus microfcopicis diffepimenta antrorfum convexa habentibus excipitur. Annon ob magnam hancce interioris firucturæ differentiam pro-

c) Saggio oritlografico orvero ofervazioni fopra le terre nautilitiche ed amonitiche della Toโcana dal Padre D. Ambrogio Solunni, Abbate Camaldolefe. In Siena 1780 . 4710. con 25 lavole. sunchmend getheilt wird, find hohl gewöllt, oder auf einer Seite erhaben, anf der andern hohl, entweder zollkonmen, oder manchmahl etwas hin und her gebogen oder gef:hlängelt.

Anmerkungswerth ift es, daß alle mikrofkopifshe Arten diefes Gefohlecits, die wir befitzen. und gefehen haben, mit vorwärts convexesz Ssheidewainden verfehen find, woron anth nicht einmuhl die doppelt kammerigen ausgenommen werden, und nur der N'autilus Ponpilius, der unter allen natiivlishen Gefihlechtszerwandten bey weiten der gröjte ift, allein vorwïts consave Scheidewcinde hat. Wir forgen: : unter deiz natürlichen, denn unter den foffilen gibt es auch lekanntlich, aber feitner, Nautiliten, die noch weit gröfler funt, aush kleinere, die aum Theil vorwarts consave Siheidewainde haben; aber nicht unter den mikrofiopifchen. Wenn von den Stü̈ke, melches Herr Abt Soldani, auf deffin Albildungen man fich ofters, obwohl wicht alleseit, verlafen kann, in feinem in italiänifsier Sprache herausgegelenen oryktographifschen Verfushe von Toscana e) auf der sweyten Platte Fig is. A. hat vorfellen lafjen, die Abbildung getreu ift, fo ift folches mus allen Jeinen Abbildungen, und fo vielen taufend hleinen Schifferchen, die wir be-

c) Saggio crittographico ovvero ofersationi Spra le terre nautilitiche ed ammonitiche della Tofoana dal Patre D. Ambrogio Soldani Aluate Canaldo-

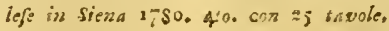


prinm ex iis condendi gemus nobis fas fitzen und gejehen haben, das einzige fuifet, faniori Conchyliologix magis Exemplar, das durch feine vorroirts hoh. periturum judicio relinquendum cenfe- len Scheidewände von den übrigen mikro. nus. flopifsien mit vorusirts convexen Scheidevänden vergehenen Arteneine Allsnahme macht. Ob wir nicht wegen diefes grofsen $U n$ ter.jihieds in den innern Ban aus dergleichen ein eigenes Gefchlecht hatten machen können, wollen wir der weiferen Beurtheilung gröfserer Konshylienkenner anheim ftellen.

Nos partim errandi metu, partim etiam, quia hoc modo omnes fpecies microfcopicæ ad novum genus pertinerent, \& Nautilus Pompilius gencris fui unicam fpeciem conftitueret, (licet hoc ordini fyfiematico non abfonum, neque plane inconfuetum effet, cum in tota natura, præfertim in regno vegetabili, quantum adhucdum novimus, aliquot. dentur genera, quorum fingula in unica tantum confifunt fpecie) ejusmodi mutationem facere non aufi fumus.

e. Hæc Diffepimenta, ati ex quibusdam fpeciebus apparet, rel in medio five centro, rel ad latus pertufa funt, \& quidem ad latus exterius five ad dorfum tefiæ, quatenus hucusque novimus, (cum contra in variis Hammouiorum fpeciebus etiam ad latus interius $\int$. centro tefta propius Sipho inveniatur). Foramen in quibusdam fpeciebus an orbiculare, aut ovale, vel integrum, vel crenatum, Sipho-

Theils aus Furcht su irren, theils anch defswegen, weil auf folche Weire alle mikrofkopifiche Arten sum neuen Gefohlecht gehiiren, und der Noutilus Pnmpilius allein eine einzelne Art feines Gefilhechts ausmashen wibrde (olggleich dicfes einer Syfematifich Verfahrungsart nicht suwider, nuch anch gan: ungevöhhlich ware, da es in der ganzen Nitur, lefouders im. Pfansenreiche, foriel daron sur Zeit lekanni ift, etliche Gefshleshter gilt, deren jedes nur ans einer einsigen Art befiehet) haben wir eine foldhe Vercindirung au machen ni:ht gewaget.

e. Diefe Siheidercände find, wie ans einigen Asten erhellet, entiveder in der Mitte, oder an einer Seite durshbohrt, und swar, foviel wiv bisher kennen, an der aissern Seite am Rücken der Schale. $D a$ es hangegen verjchiedene Arten vou Anmonshorneriz (Hammonimm) gibt, wo das Lo:h und der. Sipho an der innern dem Mittelpunkt der Sihale niihern Seite fish befindet. Diefes Losh, das an einiger 
ni pro apertura eft, in aliis, uti faltem nobis perfuafum habemus, Teftæ pro orificio. Ifta apertura quandoque (in Nau. tilo Pomp.) producta eft in tubulum tefaceun vel cylindricum, rel fubinfundibuliformem, vel moniliformem feu nodofum, nodulis longitudinaliter ovalibus. Ejusmodi tubulus, quem tubulus cutaceus tenuis (a quibusdam nerrus, item cauda animalis dictus), transcurrit, Sipho audit, qui vel continuus eft, vel interruptus, hoc eft, quafi ex pluribus Siphumculis five fingulis cujusvis ifthmi tubulis mox diftantibus, mox contiguis, tubulo cutaceo, totam teftam permeante, inter fe comexis, compofitus.

Equidem fatendum, ex voto nobis nondum fuccelfifle, ut in exemplaribus microfcopicis diverfimode diffectis, ne quidem in Nautilo Calcari, cujus Diameter tamen ad tres adfcendit lineas, $\mathrm{Si}$ phonem proprie talem, tubulum fcilicet teftaceum, plus minusve elongatum, licet adhibitis bonæ notæ microfcopiis, detegere potuerimus, qualem Gualtierus in Nautilo crifpo \& Naut, (nobis Hammonio) Beccarii fefe vidiffe prætenderat, in cujus fidem alii fcriptores hanc
Arten entweder kreisrund, oder oval, entweder gans, oder gekerbt if, dienct der Saugröhre sur Öffnung, bcy andern, wie wir wenigftens uns lereden, vcrtritt es die Stelle der Schalenmündung. Diefe Öfnung ift manchnahl(beym $\Lambda^{\top}$ ant. Pomp.) in eine fchalige Röhre verlangert, die bald walsenförsnig, bald einigermafsen trichterförmig, bald finnurförmig oder knotig, nach der Lainge mit ovalen Knoten oder Gliedern verfehen ift. Ein folches Röhrchen, durch welshes ein häutiges sartes Röhrchen (von einigen der Nerve, auch der Sshroans des Thiers genannt), durihlauft, hrifst Sipho, Saugrolire, die entweder aus einem Stiicke beftcht, oder at. gefetst, das ift, gleichfant aus mehrern Kleinen Röhrchen sufiammen gefetat ift, davon eine jede Siheideswand ihr eigenes hat, die baid abfiehen, bald an einander fofsen, und mattelft des häutigen die ganze Schale durchlanfenden Röh whens unter fich zufarmmen haingen.

Es hat uns wwar, wie wir gefteher miijfen, nach unferms IV unf:he noch nicht gelingen wollen, of wir uns gleich felr guter Vergröferungsglifer bedienten, dafs wivir in mikrofkopifchen nach - verfohiedener Riolntung durchfonzittenen Exemplaren, wicht einmahl beym Spornfshiffer $N$. Calc.), deffen Durchmeffir doch auf 3 Lin. hinan fteigt, einen eigentiohen Sipho, nehnlich eine fohalige mehr oder weniger verlaingerte Rähre haben entdesken können, fo wie Gualtieri in Nirut. crifpo und Bescarii, 
vifionem repeticrunt. Ratio, cur hic tubulus oculis evadit, fine dubio in hoc quærenda eft, aut quod per totam tefam non nifi cutaceus fit, ideoque cum in tam exili tefta etiam fubtilifimus efe debeat, una cum incola mucilaginofo brevi tempore extra aquam marinam mortuo ftatim exficcetur \& dispereat; aut quod, fi forte teftaceus eft, ob exilitatem fuam inter laborem, quo telta ad dimidiam partem cote atteritur, facillime perdatur, quod unico ductu fieri potef, vel fi nimis parum adfricatur, ubi terra \& limo occultatur. Nomifi foraminula diflepimentorum interdum marginata in quibusdam fpeciebus, non in omnibus, vifui fefe obtulerunt noftro. Hunc tubulum in pluribus, forfan in omnibus minutis, non teftaceum, fed mere cutaceum five nervofum elle, vero fimillimum eft.

f. Ad ORIFICIUM, fen domiportam, ut aliis nomen placuit '), quod attinet, obfervanda pra ceteris adluc veniunt hæc: Eam teftæ pastem, q1ıæ in latere anteriore ultimum thalamum

d) Klein Method, oftracolog, pag. 2. §.5. not, (A), einen gefehru su haben vorgegeben hat, suf deffen Trene and Glauben andere Sclniftfteller diefes eingebildete Dafeyn nacligefagt haben Die Urfache, warum diefes Rölirchen fo wonig fichtbar wird, nag ohne Zwcifel darin liegen, dafs es entweder durch die ganze S:hale hindureh blofs hiutig ift, und defsivegen, weil in einer fo kleinen arten Schale es aush äusserft feinfeyn muss, es augleich mit dem Schleimigen in kur aer $Z$ eit aufser dent Meenuaffer ferbenden Eimwohner gleich vertrocknet und vergehet, oder darss es, wenn es etwa havtfchalig ift, reegen feiner Feinheit, währendor Arbeit, wemu man die Soliale bis $\approx u r$ Hailfte anfohleift, fehr. leicht verdorben wird, welches auf cinen einsigen Zug gefchehen kann, oder auch, wenn fie $\approx$ wenig angefshliffen ruird, wo es in der Erde und Schlamm werfteckt bleibt. Bloss die kleinen Lócher in den Scheidewünden, mit einen Rande marschnahl eingeforjet, haben wir in einigen Arten, nicht in allen, erblicken können. Ann wahr.fheinlichften kommt uns vor, dafs diefes Röhrehen in den meiften, wo nicht in allen mikrofkopifehen Arten, nicht hartfchalig, fondern blofs häutig, oder nervig fey.

f. In Anfehung der MÜNDUNG oder der Hausthiire, wie einigen diefer Nahme beliebt hat d), konmit z'or andern noch folgendes $\approx u$ bemerken. Denjenigen Theil der Schale, welcher bey allen Arten

d) Klein Meth, ofracolog. p. 2, S. 5. Not. (a). 
extimi anfractus in omnibus fpeciebus occludit, (excepto N. Pompilio, cui ultimus thalamus antrorfum apertus eft), \& diffepimentis parallelam, vel fi mavis, ultimum parietem transverfalem, nominamus Plsnum orale feu finale, quia in ejus centro, vel angulis, vel ad alterum latus orificium fitum eft, quod pofierius non confundendum cum illo plano orali, uti quandoque ab aliis, ipfoque Limao in quibusdam fpeciebus, ex. gr. in N. crifpo \&c. ex minus attenta infpectione factum eft. Orificium Nautili nunquam ef totum Planum claudens ultimum articulum $f$. thalamum, fed tantummocio pars ejus aliquanta varia forme. Illud ita dictum Planum autem nunquam per-

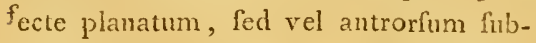
convexum, vel fubcarinato - elevatum (Nautili Calcaris var. roftrata), mox plus minusve obtufe fublunatum, mox acute fnblunatum, mox fribcordatum.

Orificium vero ipfun vel fimplex, \&: quiclem hoc vel cordatum eft, uti in Fant. Fompilio, nbi tota apertura ultimi thalami pro ore, vel lanceolatum (Naut. repandus), vel lineare e) parabolice arcuatum \& fimplex (N. Pompilioi.

c) Linseare, id quod parallelum feu latitudine ae. quali ; fic Linuss, (Philof, botan.), Giefeke (Ter. ain. bol, Lian,), Jaçui:y (Planzenkenntnils) an der vordern Seite die letate Kammer der ciussiplen Windung verfihliegt (den A'aut.Pompilins ausgenommen, deffen let:te Kanner rorwcirts offen fiehet), und mit den Siheidewcinden parallel ift, oder, wenn anan lieberwill, die lctzte Querwand, nennen wir die Mündungs- oder. Endfläche, weil in deffen Mitteipunkt, oderWinkelu, oder Scite, die Mündung fich befindet, welche mit gedaihter Minndungsfiche keinesaces vernuenget werden darf, wie es nanchnuahl von einigon, ja von Linné felbft, bey einigen Arten, ะ. B. leym Nout. crifpus etio aus $\approx u$ wenig forgfiiltiger Beobashtuing gefshehen ift. Die Mündung des Schiffer's ift niemahls die gañe Flache, die das letate Glied oder Kanmer ierfinliefit, fondern nur ein gewiffer Theil durfelben Fliche won werforiedener Gefalt. Gedachte fo genannte Fläche ift' aber nienahls wollig fach, fondern entweder vorwärts etwas contex, oder ctwas hielfüm:g erhation (N.calc. var: rofrata), bald melir oder weniger fumpf mondförnig, lould augeppitat mondförmigु, bald etwrs heraformig.

Die Mïndung aber felbft ift entweder einfuch, unt fol-henfalls bald heraformig, wie beyn N. Pomp., wo die ganze Öf: nung der letwten Kaniner ftatt der Miindung gilt, bald lansenförmig ( $N$. repundus), oder frichformig (glich breit) c) pav

e) Lineare ift nasch Linné fo risel als von gleichici Freite, parallel. In diefem Verfiande nimmt er diefs arors (Philos, bosano), aubb Giefeke (Termizs. 
des, afterizans, firigilatus $\mathbb{S c}_{\bullet}$, vel compofitum ex pluribus foramimlis $f$. punctis pertufis, aut aggregatis (N. coflatus), aut diftantibus (N.- ambiguus), mox in plano orali ad latus interins transverfe feriatim dispolitis (N. crifptis, $F_{a l}$ ba), vel per totam longitudinem plani oralis ferie fimplici ordinatis (N. planatus), mox per aliquam partem dorfi difperlis ( $N$. angulatus, aduncus), porro vel in fronte plani oralis, vel laterale feu in alterutro tefia latere, inde aut dextrorfum, aut finiftrorfum, \& quidem modo in eadem fpecie folummodo dextrorfum (Nautili plurimi), modo folum finiftrorfum (Nautilus repaudus), mox a dextro, mox a finiftro latere (Nantilus Spengleri \&c.)f). Denique orificium eft vel obtufum, rel truncatum, vel pro-

aliique, qui cum terminologia Linnaeana familiares funt eamque applicant, ifta voce utuntur eamque germanice frichfirmig, gleichlreit veriunt, et hos requimur. Ad evitandam om. nem finifram interpretationem et in quorumdam lectorum gratiam linc annotari neceflum duximus, quoniam obfervavimus, a quibusdam alias eruditis Viris praedictum terminum in alio fignificatu fumtum ct mox per gerate, mox per ananicrbrochen live in einem fortgehent ger. manice redditum effe, cum tamen fecuadum Linnaeum prius idem ac rectunt frictsm, et pofterius continusm f. non interriptum, nunquam lineare fignificet. Hocce lineare veru modo rectum, modo diverfinode arcualum, el mox la. tius, mox anguftitss elfe poteft.

f) Ex modo dictis facile colligi poteft id, quod in teftis, quorum latera in hoc genere plurimum lunt equsdem formae, fub Anfractabsscextrorjis et finilrorfis antelliganus, item quod la. rabolifci gebogen, wnd einfoch (N. Pompilioides, afterizans, fivigilatus etc.) oder aufanmen gefetst aus mehrern entweder aufammen gehciuften (N. coftatus), oder entfernten ( $N$. ambiguus) durchbohrten Puncten oder kleinen Löthern, bald muf der Mindungsfiche an der innern Seite nach der. Quere gereihet ( $N$. crifpus), oder iibcr die ganze Linge der Mindungsflishe in einer einfachen Reihe geordnet (Nout. planatus), bald iiber einen Theil des Ruackens zertheilet ( $N$. angullatus, adunius); ferner entweder rom auf der Mlundung sfichite, oder-Jeitwarts nchmllich auf einer von beyden Seiten der Schale, daher entweder veshts oder links, und suar buld in eincr und eben derfelben Art bloß rechts (die meiffen), bald blos linkts (N. repand.), oder in eben der Art

bot. Linn.), forequin (Tfanzenkenninif) und andere, die mit des inneigh hen Terminologie wer trest: fint wnd fie aruenden, gelen es auch in Deutchen darch ltrichfürmig oder gleichbreit, whi diefen folgtz wir. Zu Verneidung aller Zweydentigkeit mul einigen Lefern $\approx n$ Cefallen haben wiv diefes liter an bencriken für nötbig bejusden, weil wir beobacblet buben, dafs won ein r.mit andern itbrigens gelehren Mannern vorged iscites Kinglwort in einems andern Verflazke genoment * milbald darcb gerade, oiler warch unurtrsbrochen, in einem forlgehend, teritextf-bt worden ift, ds doch nacb Linné das erpere foviel als das lateinif be rectum oider ftrictum, oder das letatece continuutn, non intermptum; aber niemabls lineare bedestet. Dief:s Strichförmige, Gleichirrile, kann indefien ball terate, buld anf ecrfabie-

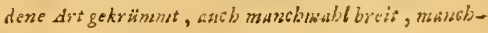
mabl folonal seyn.

$D_{2}$ 
dictum fire prominens. Etfi orificium bald auf der rechten, bald anf der linken tefiac cum Siphone neutiquam confundenSeite ( $N$. Spengleri etc.) f). Endlich ift dum elt, tamen maxime verofimile nobis die Miindung entweder fumpf, oder abgevidetur, in quibusdam fpecicbus, in qui- ftutat, oder vorftehend. Obgleich die bus nullum orificium particulare detege- Mindung der Schale mit der Saugröhre re potuimus, ifud foramen, quod in keineswegs zu vermengen iff, fo komnt ifhmis prioribus f. interioribus orificium es uns doch fehr wahrfcheinlich vor, duss

tus nobis fit fuperists, quod inforius. Si leftam nimirum ita confideramus, ul axem pro colu. mella, uti in reliquis fpiralibus, affumamus, iftud latus, in quo orificium confpicitur, infegius nominamus, latus vero oppofitum, quod

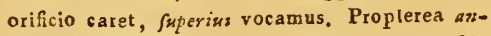
fractus dextroys fo dextrorfum ducti nobis funt, li in latere orali deorfum verlo orificium ejus faux (cavitas) infpici liceat (leu verfus finiftram vertatur).

Anfraelus finiftrin, finifirorf, contyarii, peroesA. finiftrorfwim ducti, funt, li crificium teftae latus orale deorfum vertentis adfpicienti ad $\hat{f}_{0}$. niffrsm elt eo modo, ut faux ejus videri queat (Ceu verlus dextram vergat.), P'er confequens tefla utrinque acquali latere, orificioque in fronte areae โ. plani oralis gaudens, neque dextra, neque finiftra effe poteft.

Anfractuum dextrorum et finiftrorum definitio (Fundam. teftac. [upra cit, germ, p. 212) a Linnaei praedecefforibus procreata, ab ipfo mutuata, et a fuccelloribus recocta, indiftincla nubis zon falisfecit.

5) Aus oben angefübrien kann leicbt abgenommen werikn, was wir bey den sebalen, die in diefers Gefchlecb: meiften Theils gleiche Seiten baher, nter rechts laufenden und links laufende :Windunger verfteben, ingleicben was bey wns die obere und die untere Seite keifse. Wenn man nebmlich die sebale fo betracbtet, dafs man die Axe für die spindel, wie in den xirigen Spivalgewundenen, annimmt, fo nennen wir diejenigs Seite, an welcber die Mündung fieb befindet, die untere Seite, die diefer entgegen gefetztc aber. woran keine Mündzing zu feben ift, the obere Seite. [Daber beifsen wir die Windungers alsdann rechtslaufende, wenn an der unterwürts geickteten Miüriungsfeite die Mlindung dem Anfchaser zur Rechten fo erfcbeinet, dafs man der Seblund (die Hohlung) feben kann, (oder dafs diefer fieb gegen die Linke kebret).

Linke, linkslaufende, verkehrte Windur. gen find, wenn die Mündung anter voriger Befehafenbeit der Sebale zur Linken erfebeinet, fo, days der Seblund gefeben werden kann (oder dafo èr ficb gegen die Rechte wendet.) Hierars folgt, dafseine Sebale, deren beyle Seiten einander gleich finl, unl die Mündung vorn arf der Mündungsfinche ficb bejindet, weiles recbts, nocb links genunnt werden kann.

Die Evklïrsug der rechten wni linken Windungen (Fundament. teftac. obcrz angef. deutjcb S. 212.) von Linne's Vorgüngern arfgebracht, von ibm entlebnet, snd von einigen feiner Nacbfolger wieder arfgetifibet, ift undentlicb wnd bat अrs defswegen nicht Genüge geleiftet. 
Siphonis eft, in iflımo ultimo $\mathrm{f}$. pariete ultimum thalamum occlidente, quod planum orale diximus, obvium fimul orificii totius tefta vices gerere.

\section{COLOR plurimorum fpeciminum,} \& credimus omnium, naturalis eft albus, $\&$ quidem opacorum niveus, pellucidorum hyalinus, in multis glaberrimis fplendore exaltatus, in quibusdam flavefcens, rel grifens, fine dubio ab inhærente limo marino, in aliis, præfertim follilibus, ochra. ceus a terra ferruginea, in qua fepulta erant, \& qua adhuc partim farcta funt.

Verficoloria a Ledermüllero g) iconibus coloratis repræfentata \& defcripta, dein a Martinio repetita ${ }^{b}$ ) non vidimus, forfan ifte color varius ex fallacia optices vitrorum ufu \& humectatione, uti multis jam contigit, irreptus.

h. PATRIA feu loca, ubi fpecimina noftra inventa funt, \& quidem eorum,

8) Mikrolcopifche Gemüths- and Augenergōtzun. gen. Tab. 8.

b) Syftemat, Conchyl, Cabinet. Tom, I, Tdb, 19. in einigen Arten, uoran wir keine befortdere Schalenniindung entdeiken konnten. jenes Loch, das in den erftern oder inne. ren Scheidewänderz die Mündung der Süugröllue ifs, in der letsiten Scheidewand, oder in der Wand, die die ganze letzte Kamnuer verfohliefst, welche wir die Miinzdungsfäche genannt haben, sugleich die Schalenziinsdung vertrete.

g. Die FARBE der meiften Stiicke, und wir glauben aller, ift ihrer Natur nach weifs, und swar, der undurchichtio gen Schneeveifs, der durchfichtigen wasferhell, an vielen Jehr glatten glänzend erhöhet, an einigen gelblich, oder graulich, ohme $Z$ weifel von den anhantgenden Meerfchlanm, an andern, befonders folflen, ochergell und bräunlich von der eifenhaltigen Erde, in welcher fie ver. grabeis waven, und sonit fit no:h aum Theil angefillt find.

Die vielfarbig fpielenden von Ledermilller g) in illuministen Kupforn vorgeAellten und befthriebenen, nachlier von Martini wiederhohlten Sticke h) haben wir nicht gefehen, und ihr Farbenfpiel hat vielleicht feinen Ursprung einem optifchen Betrug, der bey dem Gebrauch der Glajer and deven Befeushtung manchem Jchon waiderfohren if, au zerdinzken.

h. Dars VATERLAND oder die Gegenden, wo nufere Exemplare ge-

g) Mrikrofkofif be Gemxiths- und Augenergëtzungen. Tab. 8 ,

h) Syfteinat. Conchyl. Cubinet. 1, Bu. Taf. 19. 
qui ftatu fuo naturali adhuc gaudent, gefunden worden find, und ssvor derjenipotiflimum funt littora maris adriatici, gen, die fich in ihrenz nativichen $Z$ uftanmediterranei, hifpanici, mauritani, $\mathrm{f}$ - de befinden, find roraïglish die Küften nus arabici \&c.; parve copiæ foffilium des adriatifinen, mittellind? fhen, fpaniautem Auftriæ inferioris varii tractus, fic fchen, manritanifhen Mceres, des araetiam Hungariæ, Tranfilvaniæ, İtalia, bifchen Meer-bufens, ctc.; einerkleinen Anprafertim prope Senam in Hetruria, sahl foffiler aber verfchiedene Gegunden Helvetiæ \&c. zon Unteröfterreich, Ungarn, Siebrntiligen, Italien, rormïglich bey Sicna in Toscana, in dir Schiveis etc.

i. MENSURA maximopere differt; inter naturalia enim, prater Naut. Pompilium, quæedam dantur tantillæ molis, ut punctum acus ictu factum oculis nudis vix palpabile non excedant, a qua magnitudine ufque ad tres lineas in diametro adfcendunt; foffilium vero differentia eft inter unam tertiam lineæ atque $3 \div$ lin. diametri.

i. Die GRÖSSE ift fehr zer.chicient, unter den naturlichen gibt es, aufser dem Nant. Pompil. einige fo aufserft lleine, dafs fie einen mit der Spitze einer Nadel genachten, mit blofsen Ange kanm fichtbaren Punct nicht uberfohreiten, son welcher Gröfse fie bis anf drey Linien in Durchmeffer hinanfaigen; der folflen $U_{i n-}$ terfshied aber ift awifchen einem Drittel einer Linie und $3 \frac{x}{2}$ Linie inn Duritmeller. 


\section{NAUTILUS POMPILIOIDES.}

\section{DER KLEINE DICKE SCHIFFER.}

Nantilus Melo Soldoni.

T A B. 2. Fig. a..,$c$.

Soldani Saggio orittogr. pag. 100, zabे. 2. fig. 16, tt. TT, VV. XX.

- Tefaceogr. T. I. P. I. p. 50. tal. 46. fig. qq.?

$\mathrm{T}_{1}$

ESTA fpiralis involita, fubrotuncla-

Die SCHALE if fchuivkelförnig ike ta, parte antica productiore, laeris, fich felb/g gewnnden und, den verlängercrafla, dorjo obtufo (Nautilo Pompilio ten Theil, wo die Miindung ift, ausgeLinnæi, ab aliis $\mathrm{N}$. nagno crafjo dicto nommen, leynahe rund nnd glänsend limilis, inde fupradictum nomen nofiro glatt, disk, wit einen funpfen Rücken. datum), ntrinque umbilico profundo;igh- Übcrhasft hat diefer kleine Nantilus nis in extimo anfractu plurimum de- mit jenenz grofsen, den Linné Pompicem, licet extus apparenter rectis, non lius, andere den grofsen dicken Noutilus antrurfun concavis, uti in N. Pompilio, nennen, denu äusern Ban nach viele Ahnfed antrorfum convexis, ficut in omni- lichkeit, und erhielt daher auch obein anbus fpeciebus fequentibus, colore carn- gefihrten Nuhnen. In der Mitte befindat lefcente per teftam albam transparen- fich auf beyden Seiten ein offener tiefer tibus; articulis f. thalamis fere plana- Nabel. Die Scheidewände, deren an dev tis; plano orali femilunari; orificio pro- äufserflen Windung gewëhntich achn fnnd xime ad latus interius lineari angu- und con culsen betrachtet gerade an feyn fto, fecundum ductum anguftioris par- Scheinen, find, wie man ans angef hliffetis dimidiæ peripheria figuræ ovalis ar- nen Stücken fehen kamn, nicht wie bey den cuato \& marginato. Hac ftructura orifi- Naut. Pompilins Linn. vorneirts concav, cii \& iflhmorum fatis diftinguitur a $\mathrm{N}$. Sondern vorwarts convex, wie es bey alPompilio, ideoque hujus pullus effe non len folgenden Arten diefes Gefchlechts der poteft, uti forfan propter formam exter- Fall ift, und foheinen bläulich durch die 
nam credere poffent monnulli. Propterea, Schale durch. Die durch die Schcidewände quod D. Soldani in allegato loco Tefta- gebildeten Glieder oder Kanmern fina cographiæ Ipeciminis fui teftam totam kaum bemerkbar erhaben, und eher ganw foraminulis minimis frequentifimis obfi- flach au nennen. Die Mündungsflüche ift tam efle dixit, talemque icone reprefen- halbnondförmig; die Mündung ift sine tavit, nos vero ifta foraminula in moftris fchmale ftrichförmige nach dem $Z$ ug des exemplaribus nou obfervavimus, dubii fchmalen Theils des halben Umriffes eines hæremus, an ejus fpecimina cum noftris ejusdem fpeciei funt, nec ne.

COLOR naturalium albus, fofilium cinereus.

PATRIA naturalium Concretiones zoophytice maris mediterranei, follilium Coroucina in Tofcana.

MENSURA $\frac{7}{3}$ linez in diametro. Ovals gekriimmte und nit einem Saume zerfehene Spalte, welche hart an der nächften inuern Winaing anliegt. Durch diefen Bau der Minndung und der Scheidewände unterfcheidet er fich auch hinlcingJiol som Nautilus Ponpilius, und kann daher keine junge Brut defelben feyn, wo fiir itm manche feiner äufsercn Form wegen halten könnten. Da $\mathrm{Hr}$. Soldani in feiner Teftaccographie von den oben angefiihters Stiick ausdriicklich fagte, es fey über die ganze Schale mit felw kleinen Griibchen cerfehen, fie aucb in der Abbildung andeutete, wir aber dergleichen an unferu mehrfachen Exemplaren nicht bemerken konnten, fo macht uns diefer Uniftand wweifelhaft, of feine and unfere Stiicke con einerley Art Seyn und Seine Abbildung hierher gehöre oder nicht.

FARBE der natiorlichen weifs, der foßfilen afchgrau.

$V A T E R L A N D$ der natiorlichen die Zoophyten-Coniretionen des mittelländiSchen Meeres, der folfilen Coroncina in - Tofeana.

GRÖSSE, der fiinfte Theil einer Linie im Durchneffer. 


\title{
NAUTILUS VORTEX.
}

\section{WIRBELSCHIFFER。}

\author{
T A B. 2. Fig. d. $-i$.
}

Soldani Saggio Orittogr. p. 99. tab. 1. fig. 12?

Teftaceogr. T. I. P. I. p. 66. tab. 59. fig, $t t$ ?

TESTa fpiralis involuta, fuborbicularis, incraflata, glabra, utrinque xqualiter obtufo-conice elevata, medio lcnifime eminentiore f. umbilico parvo vix percibiliter prominente, dorfo obtufe carinato; ifthis antrorfum convexis, colore cærulefccinte per teftam tranfparentibus, angufiis, fpiraliter valde arcuatis, fere femicircularibus, articulis f. thalamis planatis, extus nomnif lineis ifiis cærulefcentibus inter fe difinctis, in extimo anfrastuum, quorum circiter quatuor funt (fig. i.), rerfus ambitum apparenter bifidis. An hrec divifo forte ifthmo breviore, quatenus ejus longitudo fe extendit, fuffulta fit, propter exilitatem tefulæ non dijudicari, folummodo prafumi poteft, prafertim quia inter terendum fuper cote nihil de illa obfervari licet. Ut clarius appareat, quomodo in hoc genere anfractus interiores ab exterioribus. obtegantur, en hujus fpeciei exemplar verticaliter apertum fig. h., ubi thalami
Die SCHALE ift finirkelformig, in fich jellft gewunden, beynahe gan: sirkelrund und glänsend glatt, auf beyden Seiten glcich ftark und funmpf kegelförnig erhaben, in der Mitte ift eine fehr fanfte, nur mit einem felir Jcharfen Vergröferungsglafe lenerkbare Erhabenheit, die einen hleinen sthroach erhabenen Nabel bildet; der Rücken ift funtf gekiert; die Scheidewände find vorwiirts gervälbt, welihe etwas blinheh durch die Schale foheinen, enge, fpiralartig, fark leynahe in einen halben Zivkel gehrionnt; die Glieder oder Kammern find flach und llofs duroh elien gedachte blantiche Linien oufsen zu unterfobiciden; in der änfserften der 3 - 4 Windumgen (fig. i) fuheinen fe gegen den Umfong an in awey Theile getheilt :u feyn. Ob diefe Theilung etwa, forveit fioh ihre Lainge erftreckt, eine durch dio dünne Sihule durclifichonde hürwere Zivifinemuand aur. Unterlage habe, laifst fich wegen der Kleinheit der Stïcke nicht 
lic tubulis depreffis fimiles, utroque latere $17-18$ confpicui funt. Fig. $g$. teftum monfrat, extimo pariete folummodo orbatam, ifthmoscute fimul novem; ex hoc numero comparato cum præfatis unbulis 18 duplo numerofioribus verofimiliter concludi poteft, quemlibet thalamum ifthmo intercalari breviore verfus peripheriam in duos femithalamos divifum elle; plannze orale anguftius acute bifurcatum, in cujus medio orificium parvum fubtriangulare angulis obtulis.

COLOR albidus, nitore margaritaceo.

PATRIA Coroncina ad Senam in Hetruria.

MENSURA , lin, diam.

Nota. Striıctura fingulari haec fpecies admodum appropinquatur in Lithologia fic dictis Helicitis (noftro N. Mammillæ \& wohl ent fheiden, fondern nur s'ermutien, sumahil da man beym Alufj:hieifen nichts davon wahrmimnt. Un deutlicher su erkennien, wie die cinfsern Windungen jederoit die innern bey diefem Gefoblechte decken, lieferia wir hier ley fig. h. eir vertical anifgefthlifienes Strick diefer Art, wo die durch dixs Auffsirieifen geöffneten, hier gedriickton Rölirchen gleichenden auf jeder Seite 17- Is Rammern $\approx$ u benterken fint. Die Figur g. aeigt cin Stiick, das blofs ron feiner ainfserften Schalenwand entblïfset ift, und wigleich neus Scheidewande. Weinn man diefe Ansahl mit den vorgedachten 18 platten Röh:chen, die doppelt ashlreicher fint, sufannmen hailt, fo kann nan warhrforeinl:cher Weife den Schlufs machen, days eine jede Kanmer durch eine kiirsere Zwifonenwandgegen den Unforng an in swey Halbkamnzern algetheilet fey. Die Mündungsfläche ift sienzlich fihninal und sugefpitst, sweygablich, in ihrer Mitte befindet fich die kleine Mündung, die wiennlich die Gefalt eines Dreyeckes mit augerundeten Winkelin hat.

FARBE, der Grundift bläulich weifs, mit einer Art von Perlmutterglanwe.

$V A T E R L A N D$, Coroncina in Tos. rans.

GRÖSSE, der dritte Theil einer Linie ins Durchmeffer.

Anmerk. Der befondere Bau sähert diefe Art in vielen denen von den Lithologen Jogenannten Heliciten (unJerm Naus: 
Ienticulari) ita, ut hanc pro eorum pro- tilus Mammilla und lenticularis), $\int 0$ daß $\beta 2$ typo habere poffent nonnulli. At fi quis vielleicht manche fiir das Urbiid derfellen eam accuratius infpiciet, \& prefertim halten wiirden; wenn man aber den Durchfectionem diametralem fig. $i$ cum Nau- finntt hior bey fig. i. betrachitet, and ihn tilo Mammilla tab.6. fig. c. \& lenticulari mit jenen des Naut. Mammilla Taf. 6. fig. eàd. tab. fig. g. item tab.7. fig. e. bene c. und N. lenticul. ebcnd. Taf. fig. g. incomparabit, moxaliter fentict.

gl. Tafel ๆ. fig. c. zufammenhiult, fo wird . man fich fogleich wons Gegentheil iilor. fiiliren.

\title{
NAUTILUS REPANDUS.
}

\section{DER AUSGESCHWEIFTE SCHIFFER.}

\author{
T A B. 3. Fig. a. b. c. d.
}

TESTA fpiralis involnta, fubovali-

$D_{\text {ie }}$ SCHALE iff fchirkelfürmig in fich repanda, lavis, utrinque valde conve- fellft gewunden, ein wenig ozal ausge$\mathrm{xa}$; dovfo fubacuto, margine tenui angu-jchweift, glatt und auf beyden Seiten fio, tamon obtufo, fubflexuofo; anfrai- fark gevöllt. Der Rïcken ift etwas fsharf; tibus finifrorfis, thalamis r. articulis mit einem dïnnen, filmalen, doch ftunzfubelevatis; diffepimentis antrorfum con- pfen Ramde ser.ehen, und etwas hin und vexis, octo in extimo anfractu confpi- her geougers. Die Windnngen laufen linhs, cuis; plano orali triangulari curvilineo fubinæuquilatero, non totam crafitudinem tefire, uti in plurimis congeneribus, occupante, fed exiftente in latere inferiore ad finisram a centro ad peripheriam fefe extendente; orifivio ad hujus latus interius fubcurvato - lanceolato a centro ad die Glieder, oder Kammen find etwas erhober, die Scheidewände vorwärts gewöibt, wovon man asht an der äusserflew Irindung suhlen kanu; die Mündungsfiäche bildet ein krummliniges, etwas unglei:rifeitiges Dreyeck, und nimnt nicht, wie bey den iibrigen Arten diefes Ge: $\mathrm{E} 2$ 
peripheriam acuminato, minutifime marginato, foraminulo rotuido minuto non nifi optime armatis oculis perceptibili, in angulo exteriore aperturæe oppolito. Utrum hoc foramen mere accidentale, an apertura fiphunculi fit, vel ob maximam pufllitatem, vel quia in unico folum fpecimine nobis obvio hoc obfervari licet, ccrto afferere non poffimus. Cum hoc fpecimen a D. Abbate Soldani una cum multis aliis ad nos miffum fit, nos nullam hujus iconem in operibus fuis in venire polle valde admiramır. Nifi iftud in Teftaceographiz T. I. P. I. tab. 36. lit. $\mathrm{X}$. icone datum exemplar in latere fuperiore pluribus uno gauderet anfractibus, propter unicum anfractum, quem in inferiore latere habet, huc referri om. ni jure pollet.

COLOK ex flarefcente albidus.

PATRIA Concretiones zoophytica maris mediterranei.

MENSURA, $\stackrel{x}{2}$ lin diam. fihiechtes, dic ganze Dicke des Stiiches ein, fundern befindet fich an der untern Seite linker Hand and reichet von Mittelp uncte lis an den Umfang der Schale. Die Mündung fellift liegt. an der innerre Seite der Miindungsficche, ift etwas krumin laizettformig, vonn-Mittelpunct gegen den Unkreis augefpitat und felir fein gerandet. Der Minadung gegen iiler. int ciufern Winkel der Mundungsfache befindet fich ein folr kleines, nur den shairfeft lewaffneten Ange fichtbares run. des Loch; ob diefis Zufall, oder die öfnnung der Nervenröhre ift, kann theils wegen der aufserften Kleinheit, theils anch weil wir es aur $Z$ eit noch nur an den einzigen vor uns liegenden Stiiske leobachten können, nicht mit Gewifsheit leftimm werden. Da wir diefes nelff irielen andern Stiickin zon Hrn. Abt Soldani erhielten, fo befrendet es uns defto mehr daron in feinen Werken keine Abbildnng funden su können. Hatte das in feiner Teftaceographie Bd. I. Th. I. Tafel 36. lit. X. aligelildete Stiick nicht auf der obern Seite nuehrere Windungen, fo könnte es vermöge der einsigen unteru nit allen Rechte hieher. geacihlt werden.

FARBE finuach gelblich weifs. $V A T E R L A N D$ Zoophyten-Concretionen des mittellindifshen Meeres. GRÖSSE: Linze inz Durchmefer. 


\title{
NAUTILUS ASTERIZANS.
}

\section{DER GESTIRNTE SCHIFFER.}

\author{
T A B. 3. Fig. e. f. g. h.
}

Soldani Teftaceogr. T. I, P. I. p. 6G. tel. 6o, lit. B.?

$\mathrm{T}$

ESTA fpiralis involuta, fubrotunda-

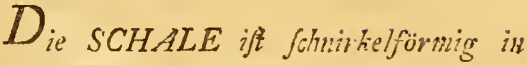
ta, fubc smpreffa, leeris, lateribus modice elevatis; dorfo acute carinato; allfractibus craflitudine repente decrefcentibus; ambilico alterius lateris aperto fatis profundo, orbiculari, protubcrantiis lanceolatis brevibus radiatim, i. e. perpendiculariter in ejus peripheria difpofitis circumdato (unde nomen fpeciei), lateris oppofiti medio loco umbilici folum leviter imprefo; articulis extimi anfractus tredecim lente decrefcentibus, verfus dillepimenta clevatioribus, cataphractæ laminas mentientibus; difepimentis antrorfum valde convexis in latere radiato coftarum infiar prominulis; planzo orali fatis convexo magno cordato; orificio ad hujus latus interius lineari, fecundum artieulorum curvaturam parabolice ar. cuato (fig. o.) lineola levilime imprella circumdato. Dni. Abbatis Soldani icon fupra citata-fecundum periphcriam teftee et diffepincuta hue pertinere videtur, fich fellyt gewunden, siemlithrund, glatt, und etwas sufammengedriickt, anf bey. den Seiten milifsig erhaben; der Riicken ift fchurf gekielt; die Windungen nehmors in ilrer Starke sienlith jühe ab; im Mittelpancte der einein Seite befundet fich einz offener, ftwrk vertiefter, kreisrunder

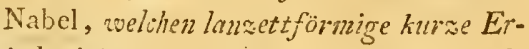
habenhoiten ungelen, die alleseit Jenkrecht auf deffelben Peripherie auffitsen, mithin die Figur eines Sternes bilden (woher auch obige Bencnnung algelcitet iff) ouf der andern Seite in fatt des Nabels nur eine Neine, Jelir Jeicht eingedriichte $V$ ertiefung. Die Glieder, deven an der ainfserffen Windung dreyzehnficht bor find, werden nur allmiihlig einwöarts kleiner, find gegen die Scheidewände etroas boul shig, und fehen drher im Gonsen wie iiber cinander liegende Schienen eines Harnifches auts. Die Scheidewände find vorwarts fark convex, wnd auf der seftirl- 
attamen deficientibus circa ambilicum ten Seite wic Klcine Rililen etwas hervorprotuberantiis nomnifi interrogative alle- ragend. Die Mündungsiliche ift ftark gegare voluimus.

COLOR flavido-grifeus.

PATRIA Concretiones zoophyticæ maris mediterranci.

MENSURA $\frac{x}{3}$ lin. diam. wöllt, grofs, und heraförnig. Die Mündumg ift firichformig, liegt ant innern Theile der Mündungsfiche, ift paralo. lifch nash der Krimmung der Glieder gebogen, (fig. o.), und mit ciner fehr feicht eingedraikten Linie ungeben. Die oben angefilinte Abbildnug des Hinn. Alits Soldani fcheint, noch deni Unififje der Sehale und den Zwifshenriunuen au urtheilen, hierher $\approx$ u gehören, doch fehlen ihr die Aernförmigen Erhabcnheiten unu den Nrabel, weeswegen wir fie auch nur fragrecife leg brachten.

$$
\text { FARDE gellich grau. }
$$

YATERLAND Zoofiyten - Concretionen des mittelländifchen Mceres.

GRÖSSE ein Drittel einer Linie in Durchneffer.

\section{NAUTILUS INCRASSATUS.}

\section{DER AUFGEBLASENE SCHIFFER.}

T A B. 4. Fig. a. b. c.

IESTA fpiralis involuta, partem orifi- Die sCHALE if jpiralförmig, in fich cii prolongatam fi excipias, fubrotundata, Jelbft gerounden, den vericingerten Theil utrinque aequaliter incraflata, pinctulis der Mündung ausgenomwen, beynahe 
excavatis notata fubtilifimis, ita ut pin- vund, auf beyden Seiten gleich und fark gendo nonexprimenda); dorfo obtufo, an- gevölbt, und durcliaus mit fo äufserft fractibus lente, fed regulariter crefcenti- kloinen vertieftciz Puncten gleichjum wic bus; umbiizo utrinque magno prominente lefiet, dafs man fie in der Abbildung undeprefo emincntiis aliquot obfoletis in- möglich ausdriicken konnte. Dor Rücken xquabili; articulis $\mathbf{1} 2$ vifibilibus paula- ift ftumf $f$ ugerundet. Die Windungen tim crefcentibus, modice elevatis; ifthmis nehmen in der Dicke langfam, aler regel. antrorfm mediocriter conrexis; plano mifsig ab; in ihrer Mitte ift auf beydon orali linato valde convexo; orificio li- Seiten ein $\mathrm{grofer}^{\circ}$, etwas erhöhter, platt ncari in latere illius interiore fecuudum articulorum interiorum convexitatem parabolice curvato, extus margine membranaceo fubtilifimo.

COLOR albide rubefcens. gedrückter Nabel wu fehen, velcher durch einige fohwache und gleichfwn verlofshens Erhöhungen rauh gemacht wird. Die 12 fichtbaren Glieder find wenig erhaben, and nehmen allnühlig aiz Grö/se au; die Scheidewände find r'orvärrts folrwach gewelbt; die Müudungsfliche ift halbmondformig and ftark erhalien gewölbt; ar dem innern Theile derfellen befindet fich die Mündung, die Atrichförmig parabolifsh wach dem Rüchen der hinein laufenden Windung gekrïmnt und mit einem feinen dannen Rande cingefafst ift.

FARBE fchrach röthlich oder weifs röthlich.

PATRIA Portus ferrarius in infula Elba mar. medit.

VATERLAND Poitoferrajo auf der Infel Elba im mittellandifchen Meer.

MENSURA $\frac{2}{5}$ lin. diam. nelger. 


\section{NAUTILUS CRISPUS.}

\section{DER KRAUSE SCHIFFER.}

T A B. 4. Fig. d. e. f. \& T A B. 5. Fig. a. b. $-$

Der Körner-Nautilus Stat. Müller. Das punksirte und mit Reifen unlegte Annoushorn Schrüter.

Das punktirte Amonshorn Schreibers.

Nautilus Arial us vulgatifinus Soldani.

Linn. Syf. wat. edit. XII. 1767. Tom. I. p. $116=. S_{f} \cdot 265$.

- Naturfystem v. Stat. Mieller, Th. 6 Bd. I. p. 356. Sp. 275.

- Syf. N. edit. XIII. Gmelin. T. I. P. 6. p. 357. Sp. ऊ.

Planc. Conch. p. Io. tab. I. fig. 2. (fals.).

Gualt. Ind. tcf.t tab. 19. fig. A. D. (fals.).

Ginanni Adriat. tab. I4. fg. I12.

Ledermüll, Microfi, tab. S. fig. B. (fals.).

Martini Conch. Cab. Bd. 3. p. 248-253. tab. 20. fig. 172, 173. (ex Gual.) Eं 174. (ex-Lederm.).

Schröter. Conchyl Kenutn. B. I. p. ro. Sp. 3 .

Schreibers Conch. Kenutu. B. I. p.3. Sp. 3 .

Soldani Saggio orittegr. p. Ioo. tab. 2. f.g. I7. Y.Z.

Teftaceogr. T. I, P. I. f. 54. tab. 33. fg. F. \& tab. 34. G. H.

1 ESTA fpiralis involuta fuboroicula- $D_{i e}$ SCHALE ift finirkclförtrig in fich ris, utrinque aqualiter fubconico-ele- felbft gerunden, wienlich kreisförmig «uvata; dorfo fubacute carinato; anfrac- gerundet, auf beydèn Seiten gleich fark, tibus tribus quatuorve (nti ex fpecimi- gleiblam kegelfömig erhaben, inden fie sibus limidiatis tab. 5. fig. $a$. $b$. videri da.llörmig rom Mittelfunste gegen dcrz 
licet) magniudline lente crefcentibus; umbitico centrali utrinque prominulo depreflo, plurimum magno, rarius minore, aliquot punctis leviter excavatis pradito, ex quo multæ ftriæ clevatiores vel coftulæ flexuofx fatis approximatæ radiatim ufque ad peripheriam tendunt, difepimentis thalamorum fuffulta, unmero autem valde impares, dum in quibusdam fpeciminibus folum viginti,' in aliis vero plures quam quadraginta, quandoque numerantur. Sulci ex iftarum ftriarum clevatione exorti lineolis incifis, brevibus, denfis, parallelis, transverfalibus feu fecundum ductum anfractum ftriati funt. Diffepinenta antrorfum fatis convexa, in extimo anfractu firiis elevatis five coftulis numero conformia. Infra iftos fulcos jacent Thalami angufti, extus ad latus anterius \& pofterius elevatiores, medio depreffiores, qui mon prorfus ad centrum pertingere videntur, quia ibi teftaceus folidus confpicitur nucleus, (tab. 5. fig. b.) An hoc folummodo in eo latet, quod fpecimina dimidiata non fatis ad medium usque cote affricta fint, propter exiguitatem certo determinari haud poteft. Planum orale fubtriangula. re, cruribus ambobus fubrectilineis, bafi parabolice incurvata. Orificium ad ejus marginem interiorem lineare anguftum, fecundum formam marginis f. dorfi anfractus interioris arcuatum, interruptum, i. e. ex pluribus foraminulis compofitum, quæ quafi iftunulis ejusdem magnitudi-
Rand su abnimmt; der Rücken if folmal wnd siemiich fcharf gelielt. Die Windun, gen, deren (wie man aus angefGhliffenen Stiicken Tab. 5. Fig. a. b. fehen kannz) 3 bis 4 fond, nehmen in ihrer Gröfse langfam $\approx u$. Anf ihrer Mitte befindet fich anz beyden Seiten ein etwas hervorflehender, aber platt gedriuckter, gewöhinlich großser, feltner kleiner, Nabel, der mit einigen finwach vertieften Puncten verfehen ift; von ihm laufen viele etwas hin und her gebogene siemlich erhabenc Streifen oder Ribben frahliveife bis an den Rand, die auf den unter ihnen ftehenden Zwifchenwänden der Kammern aufliegen, an der Zahl aber Jehr ungleich find, indem man an einigen Stücken nur ley zwansig, und an andern manchnahl einige wier sig sühlen kann; dieje Streifen liegen daher inmer nahe, oft aber forr cnge beyfonmen; die awifshen diefen befundichen Furchen find durch nahe bey einander liegende kleine, laingliche, vertiefte, parallele Puncten, oder kleine vertiefte Linien nach der. Quere, d.i. die dem Gange der Windungen folgen, geftreift. Die Scheidewände find vorwiirts aiemlich ftark convex and anf der erften Windung foviel an der Zahl, als erhöhete Streifen darauf zu zïhlen find. Unter gedachten Furchen liegen die Kammern, die ausen anf der vordern und hintern Seite etrvas erhöhet, in der Mitte etwas eingedriickt find, und nicht bis zum Mittelpuncte au reichen foheinen, inden man in der Mitte einen fohaligen 
tudinis interfepta funt (tab. 4. fig. vollen Kern benerkt (tab. 5. fig. b.) Ob f. o.).

diefes nur wou dent hervilirt, da/s die Stiicke wicht genugfann aufgefihliffen fand, lifst fich wegen der Kleinheit weder nit Gewisheit bejahen, noch zermeinen. Die Mündungstläche macht einigermafen ein Dreyeck, deffen beyde Schenikel faft geradlinig find, die Grundlinie aber parabolisch einwärts gebogen ift. Die Mündung befindet fich an innem Rande derfellien, ift frichförmig, fehr filmal nach der Geflalt des Randes oder des Rückens der innern Windung gekriinmt und unterbrochen, d. i. fie beffeht aus mehreren kleinen Löchern, die durch eben fo grofse Scheidewände von einander unterfchieden find (tab. 4. fig. f. o.).

Cum quidam celeberrimi Conchyliologi \& Lithologi in hac fpecie protypum naturalem cujusdam fpeciei minoris a Joanne Gesnero primum fic dictornm $\mathrm{He}$ licitarum fub Nautili lenticularis nomine nobis venientis (vid. infra tab. 6 . fig. e. Da es beriihnte Konchyliologen and Lithologen gibt, die in der Meynung ftehen, in diefen Nautilus das natirliche Original an einer kleinern Art der von Foh. Gefsner fo genannten Heliciten, wnferm Linfenf-hiffer (fiehe unten tal. 6. f.g. h. \& tab. 7. tota), fe invenife credi- fig. e. f. g. h. und die ganie tab. 7.), gederint; etiam hujus intuitu fpeciminis di- funden su haben, fo wor unfers Erachmidiati iconem dari aliquale operæ pre- tens es fchon in diefen Betrachte der Mïtium elle duximus, ut tam ex compara- he werth, ein aufgefoliffenes Stïck dietione internæ ftructura hujus fpeciei \& fer Art abzubilden (tab. 5. fig. b.), unn amborum Helicitarum (tab. 6. fig. $g$. \& durch Vergleichung. Jorohl diefes Auff-hliftab. 7. fig. e. $f$.), quam externæ Itructu- fes undjentes der beyden Heliciten (tab.6. ræ, non quidem magna, attamen fem- fig. g. und tab. $\%$. fig. e. $f$. ), als auch der per efrentialis elucefcat differentia. äufsern Geftalt, dens wwar nicht fturken, doch innuer wefentlichen Unterfchied deutlicher wu machen. 
COLOR albus, fæpe etiam flavefcens, aut cinerafcens, umbilici eminen. tis cærulefcens.

PATRIA naturalium precipue mare adriaticum \& mediterraneum; folilium varii trastus Hetruriæ.

MENSURA media circa $\frac{\vdots}{3}$ lin. diam. At inveninutur etiam a $\frac{1}{5}-\frac{4}{3}$ lin. Craffitudo ad diametrum latitudinis eft ut 2 ad 5 .

Nota. Siphonem fenfu firictiori, qualem Gualtierus fe vidiffe perhibuit \& ico. ne repræfentavit (Ind, teft. tab, 19. fig. d.) cujuscuue auctoritate feducti alii hoc relatum retulerunt, vitrorum fat bonorum ope videre non potuimus, quare de ifius forma fituque haud quidquam dicere poffumus. Forfan Gualtierus unnm ex iftis foraminulis orificii tefte aperturam fiphunculi effe credidit reliquaque etiam adeffe fibi perfuafit, ac præterea tota ejus icon fufpicioni obnoxia eft ejusque defcriptioni contradicens, cum ex. gr. dorfum obtufum f. rotundatum icone repræfentetur, attamen valde acutum \& marginatum fuperficiemque tefize granulis minutis obfitam defcribat, quie vero non puncta elevata feu gramula, fed excavata elongata, vel potius lineolas incifas breves habet. Hæc defcriptio fortaffe Statio Müllero in fyfiematis Linnæani verfione germánica, abfque ulteriori examine, hanc fpeciem Körnernau-
FARBE gevöfinlich weifs, fie fillt aber auch oft in das Gelbe und Grase, der erhöhete Nabel ift et zoas blauliti.

$V A T E R L A N D$ der natiorliches huntptfichlich das adriatifiche und nittcl. lindische Meer; foffil findet man fie in mehrevin Gegenden zon Tofsana.

Dic mittlere GRÖSSE ift ungeführ ${ }_{3}^{2}$ einer Linzie in Durchmeffir, man findet fie aber auch von $\frac{3}{3}$ bis $\frac{4}{3}$ Linien. Die Dicke serhilt fich aun Durchmeffer ihrer Breite ungefuih wie $2-5$.

Anmerk. Eine eigentliche Nervenröhre, wie fie Gualtieri gefehen haben soill und"abgebildet hat (Issd. teft. tab. 19. fig. d.) und welches andere durch fein Anfehen verleitet ihn na.hgefagt hulben, haben svir mit menemVergrö/serungsgliafern nicht gevoahr werden kömien, dahler wir von Seiner Gefalt und Lage wishts fagen kön. nen. Vielleidht hat Gualticri eines von den kieinen Lïshern der. Windung fiir die Öffunag der Nervenrohre angefchen und fish das iibrige hinwugedalht, wie dem Jeine ganze Figur werdiohtig ift, die Jeiner Befshreibung viderfiritht, da er $\approx$. $B$. den Rand bauchig allilden lifst, und ith do sh fein . Schurf und gefaumt, und die Oberflashe der Sstrale als feinr fen gehörnt befoln eibt, die gleichwohl keine erhabene, foudern vertiefte lingliche Puncte, ader eigentlich eingefchnittene kleine Linien hat. Diefe befshreibung mag auch dem Statius Muiller Anlays gegeben hoben, in Seinem verdeutfGhten linnzeifchen $\mathrm{F}_{2}$ 
tilus (Nautilum granulatum) denominandi anfam dedit, quam denominationem quìdem, licet inadaquatam, tamen multis Germanis jam notam \& a quibusdam receptam hic retinuifemus, quia fecundum Linnæi regulas (non ad ejus exemplum) non fine necellitate nomina jam trita mutanda funt. Sed cum jam Nautili nomen germanica voce Schiffer expreflerimus \& fupra dicta denominatio Mülleriana nondum ubique recepta fit, infuperque ad falfas ideas feducere poffit; fatius duximus totum nomen immutare novumque ac latina denominationi magis refpondens condere, etiamfingenue confitemur, ne hoc quidem nobis fatis fpecificum videri ad hanc fpeciem a ceteris omnibus perfecte difcernendam. Qui fcimnt, longe facilius effe perfectam componere fpeciei defcriptionem, quam in genere panlulum prolixo invenire nomen fpecificum adrequatum, facile nobis condonabunt.

Magis convenienter a Dno. Schrötero hac fpecies nominabatur das punctirte und mit Reifen umlegte Anmonshorn (Cornu Ammonis punctatum \& cingulis circumdatum ), etfi boc quidem non purum nomen, fed jam aliqualis defcriptio eff. Character fpecificus a Linnæo voca-
Naturfyfem, ohne weitere Unterfuchung, diefer Art den Namenz Körnernautilus beyzulegen, den wir, ob er fchon wicht palfend ift, ihr dennosh hier, als einen fchon rielen Deutsihen bekannten und von einigen angenomnenien, gelafen hätten, weil man nash den Regeln des Linné (nicht nach feinem Beyspiele) bekannte Nahmen uicht ohue Noth abindern foll. $D a$ wir aber fohon den lateinifchen Nahmen Noutilus deutfoh durcí Schiffer aus.. gedriickt haben, und die zorgenannte niiillerijche Benenunung nicht durchgehends angenommen ift, auch über diefs $\approx$ un folfchen Begriffen verleitcn liann; fo haben wir fiirs befste gehalten, den gansen Nahmen zu cundern and einen neuen mit dem lateinifchen mehr übereinftimnenden dafür zu Jetsen, ob wir wohl bekenzen miiljen, dass unch diefer uns nicht Jpeviffsh genug ift, un diefe Art ron allen Gefohlechtsierwandten vollkonmen wu unterfcheiden. Wer weißs, dafs es weit leichter fey, eine ganze Befchreibung einer Art zu machen, als in cinem etwas weitlauftigen Gefchlecht einen guten Speciff chen Nahmen su einer Art au finden, wird es uns leicht zerseihen.

Viel treffender nannte fie Schröter dos punctirte und mit Reifen umlegte Ammonshorn, obwolnt freylich diefes kein blofser Nahme, foudern fchon eine halbe Befchreibung ift. Limné driickte den Jpeciffchen Charakter lateinifch recht gut durch crifpus aus; denu crifpum und 
bulo crifpus non inepte expreffus eft; crifpatum heifst nach feiner Terminologie crifpum enim feu crifpatum fecundum foviel als flexuofum, hin und her gebocjus tcrminologiam idem fignificat, ac gen, gefchlängelt, tefta lineis crifpata, flexuofum, hin wnd her gebogen, tefta limeis crifpata i. e. lineis flexuo/is exafperata (vic. Murray fund. teftaceol. germ. in Schröteri Ephemeridibus fïr die Litter. und Kemutn. der Naturgefohichte Sंc. T. 1. p. 229. Tefta. Minus accurate Linneus ufus eft phrafi: geniculis crenatis, quibus aliquantum deprefliora $\&$ lineolis incifis transverfalibus notata thalamos obtegentia, intervalla inter altiores ifthmis fuffultas ftrias $\mathrm{f}$. coftulas intellexit. Lineolas iftas transverfales crenas nuncupanti, propter aliqualem inter utrasque finilitudinem, quidem non contradicturi fumus; fed quod modo dicta intervalla appellaverit genicula, nimis alienum abs re nobis videtur, quin etiam definitioni Geniculi ab ipfo datæ (Murray fuudam. teftac. Schröter cit, T. 1. Ephem. p. 2I5.) contradiceret; geniculum enim fecundum ipfum eft coarctatio anfractuum refpon. dens fepimento interno (i. e. quæ fuffulta eft fepimento fubjacente), ergo non refpondens thalamo, qui diffepimentis interjacet. Plerumque thalami plus minusve elevati exque partes, infra quas diffepimenta latitant, profundiores funt, \& ad hasce quadrat definitio limiana; rarius autem inveniuntur hæ partes altiores thalamis, velut in noftra fpecie, quo cafu, fi ejusmodi partes elevatiores appellarentur genicula, contra regulam i. e. lineis flexuofis exafperata, (S. Murray fundam. Teftaceolog. etc. Die Terminologic deutfich von Schröter in Litter. und Kenntu. der Nat, G. Sic, I. Bd. S. 229. Tefta). Minder richtig bediente fich Limé des Ausdrucks: geniculis crenatis, wodurch er den etwas niedrigen und quergeftrichelten, die Kanmern deckenden Raum, zwifchen den höhern iiber die. Scheidewände hinlaufenden Streifen verftund. Dawider, durs er diekleinen Querfriche mit dem Nalmen Kerben belegte, wollen wir, wegen einiger Ählichkeit avifchen beyderley Geftalt, eben nichts einwenden; allein diefe Zwifchenräume genicula su nemen, möslite sul hart Jeyn, wider.präche auch feiner. Erklärung rom geniculum (Murray fundam, teftac. Schrö. ter in angef: Bd.S. 215.), demn diefs ift nach ihn eine Verengerung der Windungen, die auf der innern Scheidewand aufliegt (Coarctatio anfractumm repondens fepimento interno), alfo nicht iiber ciner Kammer, die swifichen Scheidewänden inne liegt. Gewöhnlich find die Kammern mehr oder weniger erhöhet und die Theile, worunter die Scheidereände Tiegen, vertieft, und anf diefe trifft die linnei. fohe Definition; feltner aber find' diefe letatern höher als die Kammern, wie hier der Fall ift, und da würde es unrichtieg Seyn, die Erhöhungen Genicula zu nen- 
evidentia peccaretur, quoniam hac ratione hrec in duplici fumerentur fenfu. Interea Limnæus forfan credidit, ftrias elevatas efle articulos; i. e. tegmina five parietes fuperiores thalamorum, et fulcos profundiores lineatos vel crenatos efic a diffepimentis fufultas partes, \& tunc omnia cum definitione fna bene conveniffent. Ceterum qnoque exempla in medio funt, qua teltantur, Limmam in defcribendis fpeciebus regulas fuas terminologicas non femper fatis fricte fccutum effe, verbi canfa: Nautilum Calcar defcripfit geniculis elevatis, N. rugofum vero diffepimentis internis extrinfecus prominulis, cum tamen vi defunitionis fua genicula non elevata $\&$ difepimenta non prominula effe debeant. Occafione data prætermittere nolumus, vocem coarctatio a Dno. Schrötero 1. cit. fenfui linnæano firictiori non fatis convenienter vocabulo Verbindung (Combinatio, Conjunctio) germauice redditam efle, quod Viro lunic laboriofillimo magnoque in fcientia conchyliologica erudito, forfan nimis feftinanti clapfum eft, ficut etiam verofimiliter conjici licct, totum non perfectam verfionem omnium terminorum technicorum, fed potius definitionum folummodo fuife. Sic etian fortafle nimis fuperficialis infpectio Nautili crifpi noftrum de reliquo æeterne venerandum Linnæum in crrorem induxit, ut planum orale, quod quandoque fere cordatum, in quibusdam fpeciminibus vero admo- nen, weil anf folche Weile dieje einen Doppelfinn haben wiirden, roelthes wider die Regel der Dentlichkeit wïre. Indeffen ift es aush üüglish, dafs Linné geglaubt hat, die erhiheten Streifen hier woven die Glieder, d.i. dic Devken oder die obern Winde der Kammen, und dic sertieften geftrichelien oder gekerbten Furchen darswifinen die :on dish Scheidewinden unterftiitsten Theile, und dann hatte or uach feiner Definition recht gehabt. OJbrigens hat man Beyfpiele, dafs Linnè bey B.f:hreibung verfolicdener Artenfeinenter. minologifohen Regeln nicht alleseit getreu geblieben ift. Z. B. den N.rutilus Calcar bes:hreibt er mit geniculis elevatis, den Nout. rugofus mit dillepimentis internis extrinfecus prominulis, da doch nach feiner Erklarung genicula nivht elcvata, und diffepinnent a nivht prominula feyn follen. Gelegenheitlich au fagen, hat Hr. Schrüter an angef. Ort das Wort Coarclatio nicht in eigentlishen linneifchen Sinn deutfch durch Verbindung (Combinatio, Conjunctio) ïberfetwet, wel:hes diefen fo arbeitfunen Manne und grofsen Konshyliologen in Eile entwifhet feyn mag, wie man denn auch iiberliaupt fiehet, dafs das Ganze keine rollkommene Überfetzung der Worte, fondern vielmehr uur der Erhlirungen hat feyn follen. So mag auch eine zu fiichtige Anficht des Nout. crifpus unfern iibrigens ewig verehrlichen Linne verleitet haben, die Mündungsfläche, die manchmahl faft her:äörnig, an andern 
dum compreffum, velmera rima eft, pro Individuen wieder ganz zufanmengeorificio ipfo habuerit \&, quia in fuo exem- driickt, oder voie eine blofse Spalte erplari partem hanc femicordatam invenie- foheint, fïr die Mïndung felbft anzufe. bat, eam hac de re aperturam femicor- hen und, weil er an feinen Exemplar diedatam dixerit. fen Theil gerade halbheroformig fand. ihn aperturam Senticordatam zu nennen.

\section{NAUTILUS COSTATUS.}

\section{DER GERIBBTE SCHIFFER.}

T A B. 4. Fig. g. h. $\dot{8}$

IESTA fpiralis involuta, fubrotundata, antice fubrofirata, utrinque valde convexa; dorfo obtufo, per medium quafi carinato, margine membranaceo tenui partim fubdentato, dentibus latis obtufis brevibus, in anfractuum utroque centro umbilico minuto parum prominente; ifthmis antrorfum leviter convexis; articulis fex confpicuis fere planis collatis, coftis anguftis, fed denfis, ambitui parallelis , excepto ultimo, cujus coftæ antrorfum in partis fubroftrata extremitate fubcrenata convergunt, nti \& penultimo, cujus aliquot coftx exteriores paraillelæ, ben finnal, aler dicht beyommen, und
Die SCHALE ift spiral in fich felbet gewunden, roru etwas fohnausenartig, fonft aber rund, auf beyden Seiten ftark gervölbt; der Rücken gevundet, und erfineint nur durch den diunen häutigen Rand, der hier und da mit einer Art diunner breiter Zihne verfehen ift, als ge. kielt; die Windungen haben auf jeder Seite in der Mitte einen kleinen etwas hervorftehenden Nabel; die Scheidewände find rorwärts nicht ftark convex; die auf der erften Windung fichtbaren fechs Glie. der find beynahe flash, geribbt, die Rib. 
interiores vero inordinate, partim quali richten fich in ihrer Lage nach dem Unifractæ, dislocatæ funt; plano orali para- lauf der Windungen, ausgenommen in bolico, in quo prope extremitatem cre- letaten Gilied, wo fe vorwairts gegen den natam partis roftrata loco orifiii adfunt fincuzenförmigen an Ende etwas getres pori approximati in triangulo difpo- kerbten Theil au sufanmen laufen, fo fiti, infra quos rimula brevis utrinque auch an vorletsten Gliede, wo etliche fubmarginata fecundum longitudinem ätssere Ribben parallel, die innern aber plani oralis incifa eft.

COLOR albidus.

PATRIA littus mauritanum Africæ.

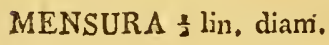
unordentlicb, whis 7 heil wie serbrochen, zerftreut liegen. Die Mündungsfläche ift parabolifch, anf welcher nahe an den gekerbten äufsern Ende des f.hnauzenähnlichen Theils anftatt der Mündung drey kleine runde Löcher in einem Dreyecke nahe beyfanmen wu fehen find, unter welchen eine feine kurse, auf beyden Seiten etwas anfgeworfene Spalte nach der Länge der Mündungsfliiche eingefchnitten ift.

$F A R B E$ weifslich.

$V A T E R L A N D$ die marokanifse Küfte in Afrika.

GRÖSSE $\frac{1}{2}$ Linie in Durchmefer. 


\section{NAUTILUS STRIGILATUS.}

\section{DER GESTRICHELTE SCHIFFER.}

VARIETAS C

dorlo integro,
ERSTE ABÄNDERUNG

mit ganzen Kiicken。

\section{T A B. 5. Fig. c. d. e.}

Soldani Tefassogr, T, I, P. I. p. 54. tab. 34. fg. 1. (fubfumil.).

$\mathrm{T}$ ESTA

fpiralis involnta fuborbicnlaris, utrinque mediocriter elevata, fed articulis tribus ultimis fatis tumidis; dorSo acute carinato integro, mbilico mediocrilævi, parum impreflo; diffepinnentis antrorfum convexis, extus elevatis feu fubcoftatis, plcrumque viginti duobus quatuorre in extimo anfractu confpicno; inde thalami angufti cxtus medio plani, ad latera fubelevati, inter diffepimenta fpiraliter ftriati, quafi crenati friis incifis brevibus confertis; plano or cli fubcordiformi, fed duobus lobis acuminatis, in cujns margine interiori orificinm linca-. wwe fohen den Scheidewcinden nach den re angufum fubparabolice fecundum for- Gonge dor Windungen mit vielcn kuran mam marginis $f$. dorfi anfractus interio- an einander iiegenden vertieften Strichen, ris arcuatum, integrum (non interruptum at in N. crifpo).
Die SCHALE ift infich fellift faft kreisrnind gerunden, auf beyden Seiten mittelmäfsig erhaben, an den drey lctsten Gliedern aber siemlich anfgefchwollen; der Rücken iff foharf gelielt mud gans; der Nabel zon mittlerer. Gröfse, glatt, und etwars eingedriickt; die Scheidewände find rorvörts conzex, auswendig gleish feinen Ribben erhaben, und gewöhlich ouf der erften Windung einige «wansig an der $Z$ ahl; daher find die Kammern enge, anstuendig in der Mitte flach, an leyden Seiten etras erlaben, gleichan wie mit Kerben befetst; die Mündungsfläche ift cinigemafsen herwförnig, die leyden Lapten oder Flïget 
nber sugefpitst; an ihrem innern Rande liegt die frrichformige fchnole Mjindung, aie etwoxs paratolifich nach der Geftalt des Randes oder fiuchens der innern Windung gelroimmt und gans ift (nicht

COLOR albidus.

PATRIA copiofe ad Poville prope Novi in littorali Auftriaco maris adriatici.

MENSURA $\frac{2}{5}$ lin. diam, unterbrochen, wie am kraufen Schiffer).

$F A R B E$ weifslich.

$V A T E R L A N D$ häufg au poville bey Novi im öfterreichifshen Kï̈flenlande ans alriatifchen Meer.

GRÖSSE $\frac{2}{5}$ Linie in Durchneffer. dorโo membranacep dentato.

\section{VAFIETAS $\beta$}

\section{T A B. 5. Fig. $f$. $g$.}

Discedit a precedente non nifi margine dorfali membranaceo dentato, dentibus acutis, inæqualis longitudinis, laxe diftantibus a fe invicem.

\section{COLOR, PATRIA \& MENSURA} ut fupra.

Nota. Multi forfan hunc Nautilum, prafertim primo intuitu, eandem cum $\mathrm{N}$. crifpo efle fpeciem credent, cui alioquin valde fimilis eft; fed differt fatis articulis ultimis tumidioribus, lateribus comprelfioribus, umbilico minori impreffo, qui in N. crifpo magutus \& prominens. Denique plani oralis differentia notabilis \& in varietate $\beta$ margo dorfalis mem.

Weicht von der vorhergehenden bluss dadurch ab, dafs iiber den Riichen ein diinnes, mit Jpitzigen, ungleich hohen und weit von einander abfehenden Ziihnen iergehenes Blättchen herunläuft.

FARBE, VATERLAND und GRÖS. $S E$ einerley nit voriger.

Anmerk. Viele werden diefen Nrutilus, voraiiglich beym erften Anblich, fïr den kraufen Schiffer, wit welchen er unffreitig vieles gemein hat, halten; allein er uniterficheidet fich hinlänglich durch die etwas gefolrwollenen letzten Glieder, durch die fächern Seiten, und durch den kleinern eingedriickten Nabel, welcher bey dem kraufen oder gef-hlangelten Nau- 
branaceus, quo N. crifpus caret. Nemi- tilus grofs und erhaben ift; endlich ift nem ergo, qui hanc fpeciem accuratius auch der Unterfehied der Mïndungsfäcontemplabit, feductum iri credimus, ut che anffallend, und bey der aweyten $A b$. eam pro fola modo dicti Nautili varie- änderung das gezähuelte Blättchen anf tate accipiat. dem Rïchen, das bey den kraufen Schif: fer mangelt. Wir glanben alfo, dajs Niemand bey genanerer Beobachtung rerleitet werden wird, ihn für eine blojse Abänderung des let:tern ansufehen.

\title{
NAUTILUS CRATICULATUS.
}

\section{DER GEFLOCHTENE SCHIFFER.}

\author{
T A B. 5. Fig. h. i. k
}

TEsta fpiralis involuta, orbicularis,

Die SCHALE ift fpiral kreisrund in utrinque admodum convexa, adeo ut fich fellft gewunden, auf beydera Seitenfehr primo intuitu compreflo-globularem cre- ftark gewölbt, fo, dafs man fre beym erderes, ni dorfo carinato, licet obtule, Aten Anblick fïr gedrilcht kugelfurmig gauderet; umbilico leviter prominulo, halten könnte, wenn fe nicht einen obpermagno; longitudinem fere femidia- wohl ftumpf gekielten Rücken hütte. Der metri tefiæ exæquante, multis punctis Nabel ragt feicht hervor, ift fehr grofs, impreflis fparfis notato; a hujus periphe- inden er beynahe die Länge des halben ra ad peripheriam exteriorem five mar- Durchmefers der Schale beträgt, und mit ginem teftæ Griis. radiantibus multis ap- vielenzerftreut liegenden vertieften Punsproximatis fubelevatis, lineis incifis fub- ten durchous lezeichnet ift; von feinent tilifimis confertis ftrias transverfim feu Unkreife laufen frahlweife bis an derz $\mathrm{G} 2$ 
margini parallelis decuffantibus (fic tefta äufsern Unfong viele fohwach erhabene fpeciem craticulatam mentitur); diffepi- Streifen nahe neben einander, die durch mentis antrorfum leniter convexis, hinc fehr feine, auch dicht an einander mit den thalanis anguftis; plano orali propter Rïcken parallel lanfende vertiefte Linien ultimi articuli f. thalami parvam promi- quer durchkseuset werden (wodurch die nentiam fatis angufto, femilunato five Sihale das Anfehen bekonnt, als wärefie fubfalcato; orificio fublineari angufto fe- korbarig geflochten). Dic Scheiclewände cuudum curvaturam articulorum fubpa- find vorwirts feicht gevö̈lbt and sienrabolice arcuato.

COLOR albus.

PATRIA fiuns arabicus, in cujus arewa conchyliofera a D. Spengler inventa fpecimina nobis mifra.

MENSURA $\frac{1}{2}$ lin. diam, lich dicht an einonder, daher die Kammen enge. Die Mündungsfläche ift wegen des wenig vorfpringonden letisten Gliedes oder Kanmer wiemlish filmal, einigernafsen halbmondförmig, oder etwas fichelformig; die Mündung ift faft ftri-hlformig, finmal, etwas parabolifih nacb der Kroinmmung der Glieder gebogen.

$F A R B E$ veißs.

$V A T E R L A N D$ der arabifche Meerlufen, in deffen Sthneckenfande vom Hrn. Spengler gefunden and uns gefendet. GRÖSSE $\frac{x}{2}$ Linie in Durchmeffer. 


\title{
NAUTILUS MAMMILLA. \\ DER BRUSTWARZEN SCHIFFER.
}

\author{
T A B. 6. Fig. $\%$ b. $6 . d$.
}

$T$

ESTA fpiralis involuta, orbicularis, lævis, utrinque mediocriter elevata (non convexa, fed depreflo-conica), mulbili. co majusculo prominulo non abrupto a reliqua tefta (uti in quibusdam aliis), fed leniter declivi; dor.o five margine obtufe carinato; anfractibus fex feptemve (uti ex fpecimine dimidiato fig. c. apparet), amplitudine lente crefcentibus; ifthnis antrorfum leviter convexis fat obliquis approximatis; thalamis itaque inguftis multis, in extimo anfractu circa $22-26$, in omnibus circiter 100 confpicuis (relative anguftioribus quam in aliis congeneribus, forma externa fimilibus.)

Die SCHALE if folmirkelförnig in fich fellef gerounden, kreismund, anf beyden Seiten mittelmijsig erhaben, (nicht gewölbt, fondewn niedrig kegelformig) und vollkommen glatt; der Nabel siemlich graßs und etwas hervorragend, aber nicht abgefondert von der iibrigen Schale (wie bey ananchen), fonden fanft ablaufer:d; der Rücken oder Rand ift fumpf gekielt. Windungen find Jechs bis fieben, (wie aus der angefelrliffenen Hailfte fig. c. erhellet), die ganz allnaihlig an Weite sunehmen; die Scheidewände fnnd romwairts fchwach convex, sienslich fchief und nahe an einander; die Kammern, deren an erften Unlauf einige wwansig, in allew aber gegen hundert find, liegen siemlich enge bey einander, (verhaltnifnafsig weit enger, als bey andern Gefhlechtsverwandten, die eine diefer Art aihnliche Geftalt haben).

De plani oralis \& orificiz ftructura nihil dicere poffumus, quippe qux partes in omnibus hucusque follilibus folum inventis a nobis vifis exemplaribus detritæ

Von der Mïndungsfläche und Mïndung kónzen wir niclits fagen, weil diefe Theile an allen bisher beobashteten Beyspielen, die fich bloss forfil gefunden haben, 
funt. Id foraminulum cuim, quod in uno alterove fpecimine oblervatur, non orificium eft, fed thalami inter eos, qui adhuc exftant, ultimi antrorfum partim detriti cavitas aperta.

\section{COLOR albidus \& flavefcens.}

PATRIA, hucusque folum follilia inventa funt fpecimina in pago Brumn am Steinfeld aliquot horarum fpatium diffito ab urbe Vindobona Aufria inferioris, ubi ex fonte in medio lacu cum aqua fcatente \& fragmentis aliorum petrefactorum proveniunt. Obvia quoque funt in aliis locis Aufrie inf. \& ad confinia Hungaria, in lapicidinis, per tota praripia fupcriora lacus Neofidelienfis difitis, nempe ad S. Margaretham, Mariam Lorettanam, Grusbach (Kroisbach) \&c., \& quidem in hoc ultimo loco una cum aliis Nautilitis \& Ammonitis minutiffimis, ex quibus \& hinc inde immenfis milleporitis (prefertim Millepora calcarca Pall.) pulchrisque pectinitis tota congeries lapidea compolita eft, que vulgo, fid perperam, lapis arenarius racatur, quia ha teftule marinæ microfcopicx ad hodiernum tempus oculo fugitivo infpectie pro puris granulis arenariis habitze \& venditate funt.

MENSURA : $-1 \frac{1}{2} \mathrm{lin}$, diam. abgerielen find; denn das kleine Loch, das an einigen Stiituen beobachtet wird, if nicht die Minndung, fondern nur die offene Höhling der vorvïrts zun Theil algeriebenen letwten Kammer unter den noch vorhandenen.

FARBE weifslich, auch gelblich.

VATERLAND von Brunn an Steinfeld, etliche Stunden von Wien in Unzteröfterreich, wo fe durch das QuellwafSer einer nitten in einem Weiher befindlichen Quelle, in Gefollfshaft vieler anderer Petrefacten-Fragnente aus denu Grunde hervor getrieben werden. Man findet ihn auch an mehrevn Orten Unteröfterreichs und auf der Grinnse mit Ungaru in den Steinbrïchen längs den obern Theil des Neufiedler Sees, all Margareth, $M a-$ ria Loretto, Grusbaih (Kroisbath), im letatern Biuch in Gefelifchuft wit Millionen der kleinften Ammoniten, woraus mit einigen Milleporiten (befonders der Millepora calcarea Pall.) auch fohönen Pectiniten, der ganse Stein befteht, den man fiilfchlich einen Sandflein nennet, weilman bis doher diefe kleine Meeresbrut obenhin für blofse Sandkörner angefchen und ausgegeben hat.

GRÖSSE I bis I I Linie im Durhmefler. 


\title{
NAUTILUS LENTICULARIS *).
}

\section{DER LINSENFÖRMIGE SCHIFFER.}

\author{
Т А в. 6. Fig.e.f.g. h, \& Т А B. 7. Fig. $a-\%$.
}

VARIETAS $\propto$.

ERSTE ABÄNDERUNG.

T A B. 6. Fig. e. $f \cdot g$. h.

$\mathrm{T}$

EST A Ppiralis involuta orbicularis $1 æ-$ vis, utrinque valde convexa, non um. bilicata, dorfo obtufe carinato, anfractibus tribus quatuorve vix fenfibiliter crefcentibus (fig. g.); hinc thalani in exemplaribus dimidiatis transverfaliter angufii \& quafi paralleli apparent, \& fi a peripheria ufque ad centrum pertingunt, cum quilibet anfractus interior a proxime fequente exteriori non a dorfo folum, fed \& lateraliter cuticularum lentis vel pifi inftar totus obtectus \& involutus fit; diffepimenta vero diftantiora antrorfum leviter convexa \& fatis obli-

*) Hace eft fpecies HelieilarumLithologorum, praecipue Jo. Gesneri, 'quorum multifaria nomina promiscue variis lpeciebus vel varietatibus a diverfis auctoribus donata ad omnem ambiguia tatem evitandam confulto praetermifimus.

$D_{\text {ie }}$ SCHALE ift filmirkelformig eingerollt, kreisrutzd, glatt, anf beyden Seiten ftark gewölbt, ungenabelt; der Rücken ftumpf kielformig; die drey bis vier Windungen nehmen gans allmühlig und kaum bentertibar an Gröse $\approx u$, (fig. g.) daher ertheinen die Kammern in hall aufges:hliffenen Beyfpielen nath der Qucre eng und gleichfam parallel, ungeachtet fie rom Unkreise bis atm Mittelpunit reichen, indenn einle jede innere Windung durch die niithl folgende ainfsere wicht blo/s an Riichen allein, fondern auch auf den Seiten, wie die Hiute (Bailge) einer

*) Diefs ift eine Art der von den Lithologen; vorzüglich von $\mathcal{F} b$. Gefsuer, fogenannten Feliciten. deren vislerley Nahnzen cinne Unterfohied bald diefer, bald jener Art oder Abüntersing von verfobiedenen Schriftjellern gegeben wuriden, die wir bier, um aller Zweydertigkeit auszwweisher, wobibediucbilicb ansgelafen baben. 
qua funt, in diverlis fpeciminibus circa Linfe oder Erbfe, gans überdeckt und $50-56$, centro intrinfeco globulari cavo eingewunden ift; die Scheidewände hinmagno. Ea pars, ubi Orificium præfu- gegen ftehen weiter voln einander, find mendum, detrita eft, ergo de hoc nihil vorwirts fchwach convex -und in siemlich dici poteft.

fchiefer Richtung in verfchiedenen Stücken etliche und funfsiig. Derjenige Theil, woran nuan die Mindung vermuther kann, ift abgerieberz, and alfo wichts divon $\approx u$ fagen.

COLOR albefcens.

FARBE reißBich.

PATRIA ad Claudiopolim Tranfylvania, follilis.

MENSURA $I \frac{x}{2}-2$ lin. diam.
VATERLAND: wirdbeyR'anfenburg in Siebcnbiirgen siemluch häufig gefundein, bisher alfo wur nosh foffil.

GRÖSSE $+\frac{r}{2}-2$ Linien ins Durtho meffer.

VARIETAS s.
ZWEYTE ABÄNDERUNG.

\section{T A B. 7. Fig. a. b.}

Hrec varietas differt folummodo a præcedente in eo, quod fuperficies ejus non lævis fit, fed granulofa, granulis confertis.

COLOR, PATRIA \& MENSURA at fupra.
Diefe Abändering weicht :on der vorgehenden bloßs darin ab, das ihre Oberficiche nicht glatt, fondern mit Kürnern dicht biget:et ift.

FARBE,VATERLAND und GRÖS$S E$ wie oberl.

\section{VARIETAS $\gamma$.}

\section{DRITTE ABANDERUNG,}

T A B. 7. Fig. c. d. e. f.

Discedit a prioribus majori volumine, Uhterf-heidet fich von den vorigen ditpluribus anfractibus, hic $8-9$. in fpe- durch, daßs fe größer ift; aus mehrerelt, 
ciminibus dimidialis, apparenter trans- hier 8 - 9 Windungen, die in den bis verfim angufioribus (fig. e.) parieti- ₹ur Hcilfte aufgefhliffenen Sticken (fig. busque proportionate crallioribus \& c.) fcheinbar finnciler find, befteht und thalamis quoque angufioribus, licet dif- ierhailtnifsncifsig dickere Seitenzonde, fepimenta difiantiora a fe invicem efle auch engere Kanmern hat, obgleich die videantur, quam latitudo anfractuum ap- Ztvifshenzwönde veiter von einander entparenter talis eft, fed diftautia minus re- fernt au feyn fiheinen, a/s die foheinbare gulari, porro centro caro minori. De re- Breite der Windungen ift. Die Entfer.. liquo convcrit fuperficic lavi cum prima varietate.

COLOR \& PATRIA, ut antea.

MENSURA $2-3 \stackrel{x}{2}$ lin. diam. unug ift aleer wicht gleich und der hohle Mittclpunlit kleiner. Übrigens konnut diefe Varietiat durch die glatte Oberfache nuit der erften iiberein.

FARBE und YATERLAND wie vorther. meder.

GRÖSSE 2 $-3 \frac{x}{2}$ Linien in Durch.

VIERTE ABÄNDERUNG.

VARIETAS 8.

\section{T A B. 7. Fig. $g$.}

Difcedit a var. $\gamma$ folummodo fuperficie ftriata, ftriis a centro ad peripheriam radiantibus.

COLOR, PATRIA \& MENSURA ut. fupra.

Geht von der dritten Alinderang b\%os durch die gefireifie Ol.erficishe $a b$, da die Streifen vom Mittelpunst aum Unkivife firahlweife auslaufen.

FARBE, VATERLAND u.GRÖSSE wie obez.

VARIETAS $\varepsilon$.

FÜNFTE ABÄNDERUNG.

$$
\text { T A B. 7. Fig. } h .
$$

Hæc etiam a varietate $\delta$ non differt, Aush diefe weicht von der vierten surch nifi granulis inter ftrias partim confertis, wishts weiter $a b$, als durch die Ehorn- 
partim fparfis. In reliquis convenit etiam chen, dic awifchen den Streifen theils in Hanfen begfammen, theils einzeln wer. freut fit:en. In ïbrigen konnt fe ouch inz Anjelnung der

COLORE, PATRIA \& MENSURA. FARBE, des VATERLANDS und der GRÖSSE damit ïberen.

\title{
NAUTILUS RADIȦTUS.
}

\section{DER GESTRAHLTE SCHIFFER.}

\author{
T A B. 8. Fig. a. b. c. d.
}

\section{Testa fpiralis involuta, orbicularis,} lavis, utrinque fatis convexa \& radiata, radiis multis approximatis, flexuofis, plurimum fimplicibus, pafim verfus ambitum bifurcatis, aliis alterne injectis brevibus, ab ambitu ad centrum tertiam circiter femidiametri partem occupantibus; dorfo obtufe carinato; anfractibus quatuor licet exterioribus majoribus, interiores plene obtegentibus, tamen latitudine apparenter lente crefcentibus (fig. c.); diffepinentis antrorfum mediocriter convexis valde obliquis $\&$ approximatis; centro intus globulari cavo; orificio detrito.

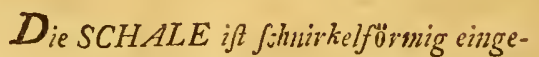
rollt, kreisrund, glatt, auf beyden Seiten siemlich erhaben und geftrahlt, die Strahlen find hin und her gelogen, häufig, nahe beyfanmen, meiftens einfach, hier und da etliche gegen den. Umkreis su sweygabelig, swifhen ihnen liegen wechSelveife andere kurae, die von Unkreis gegen den Mittelpunct an ungefihr den dritten Theil des halben Duruhmeffers ausmacheu; der Rücken ift ftumpf gekielt; Windungen find vier, welche, ungeachtet die innern durch die ciufsern gröfiern völlig bedecht werden, doch fihein. bar gans allmahlig an breite sunehmen 
(fig. c.) $\dot{j}_{1}^{\prime}$ die Scheidewände find vorwärts Sihroach gewölbt, fehr fchief und nahe an einander; der Mittelpunct ift inwendig kugelformig und hohl. Die Mündung ift abgerieben.

COLOR flavo-albefcens, radiis fubcxrulefcentibus.

PATRIA finus arabicus; fpecimina a Dno. Spengler ex larena cochleas majores replente eruta funt, cujus bonitati noftra debemus.

MENSURA \& lin. diam.

FARBE gelblich weiß, mit etwas biculishen Strahlen.

VATERLAND der arabifche Meerbufen, die Exemplare davon find vom Hrn. Spengler im Sande, womit gröjsere Schneckenfichalen angefiillt waren, gefunden worden, deffen freundfchaftlicher Giite wir die unfrigen an ierdanl en halen. GRÖSSE 1 Linie im Durchmefer.

\section{NAUTILUS VENOSUS.}

\section{DER GEADERTF, SCHIFFER.}

T A B. 8. Fig. e. f. $g . h$.

$\mathrm{T}$

EST A fpiralis involuta, orbicularis, lævis, utrinque fatis convexa, radiata, radiis fat difantibus, (remotioribus ideoque paucioribus, quam in N.radiato), flexuofis, plurimum fimplicibus, hinc inde verfus am. bitum bi- \& trifurcatis, nullis brevioribus intercalaribus, (quo etiam difinquitur a $\mathrm{N}$.

Die SCHALE if f-hnirhelf urmig eingerollt, kreisrund, glatt, auf jeder Seite zienlich gewülbt und geftrahlt, die Strahlen find und her gebogen, aiemlich von einander abfehend, (entfernter und folglich weniger, als anz geftrahlten Schiffer), meiften Theils einfuch, hier und da gegen 
radiato); dorfo obtufe carinato; anfiuzti- den Unikreis wu swey-anch dreygabelig, bus quatuor apparenter lente crefcentibus ohne kume Zwifihenftrahlen (wodurch (fig. g.); ifthmis quoque fatis diftantibus fich dicfer auch rom geftrahlten Schiffer (duplo remotioribus, quam in N. radia- unterfcheidet); der Rücken if fumpf geto, minusque obliquis, quam in illo), an- kitlt; die vier. Windungen nehmen foheintrorfum convexis; centro interno medio- bar langfan an Weite $\approx u$ (fig. g.); die cri globulari cavo. Orificio detrito.: Scheidewände ftehen anch siemlich von cinander (doppelt weiter, als an geftrahlte $n$, und weniger fchrage, als an jenem), und find rorwirts gewolbt; der inwendige Mittelpunct if won mittelnaißiger Grojse, kugelig und hohl. Die Mündung: if abgerieben.

COLOR, PATRIA \& MENSURA, FARBE,VATERLAND undGRÖStill in N. radiato. $S E$ wie an geftrahlten Schiffer.

Nota. Primo intuitu hæc cum Naut. radiato eadem fpecies elle videtur, fed ex præcedente defcriptione patebit, diverfas inter fe efle fpecies. Forfan nonnullis dure follummodo varietates unius fpeciei efle videbuntur.

Aumerk. Bey denu erften Anblick fcheint diefe Art mit dem geftrallten Nautilus einerley an feyn; allein aus vorgefetzter Befchreibung wird erhellen, dafs es swey verfohiedene Arten find. Vielleicht wird es manchem :orkonmen, daß $\beta$ es nur wovey Abänderungen von einerley Art Seyn. 


\title{
NAUTILUS STRIATO - PUNCTATUS.
}

\section{DER GESTREIFT - PUNCTIRTE SCHIFFER.}

\author{
T A B. 9. Fig. h. i. k
}

1 ESTA fpiralis inroluta, fuborbicularis, globofa, umbilico tutrinque mediocri plano, fuperficie extimi folum confpicui anfractus radiatim firiata; friis mediocriter diftantibus, lineolisque incifis fubliliffimis fpiraliter parallelis firias transverfim decuffantibus, denfe intermixtis, multis punctis imprefis; dorfo late rotundato; thalannis \& diffepimentis antrorfum mediocriter convexis, circa $20 \mathrm{in}$ extimo anfractu confpicuis, tot quot firia; plano orali femilunato levi; orificio lineari angufo fubfemicirculariter fecuudum craflitudinem anfractuum arcuato.

COLOR albus.

PATRIA finus arabicus; a Dno.Spengler in cochleis majoribus inventa fpecimina.

MENSURA $\frac{2}{5}$ lin, diam.

Die SCHALE ifl fpiralartig cingerollt, faft hreisrund, kugelig dick, wit einem mittelnäfsig grofsen flachen Nabel anf jeder Seite, die Oberficith der allein fichtburen äufserfien Windung iff frahlig geftreift, die mittelmalsig weit won einander abfehende Streifen werden durch viele hleiné, Jehr vertiefte, mit den Windungen parellel lanfende Linzien quer durchhreuset, auifinen welchen viele hleine arertiefte Puncte dicht zerflreut liegen; der Rücken ift breit algermudet, die Kammern und Scheidewände find vorwörts mittelnuifsig gewölbt, an der äusserften Windung bey 20 fichtbar, eben fo viel, als Streifen; die Mündungśfläche ift halbmondförnig und glatt; die Münduns frichförnig, enge und in Gefalt eines halben Zirkels nach der Dickie der Windungen gebogen.

FARBE weiß.

$V A T E R L A N D$ der arabifshe Meerbufen, aus welchem die gró/seren Sihnechen find, in deren Hohlungen Hr. Spongler dieje Stïcke gefunden hat.

GRÖSSE $\frac{2}{5}$ Linie in Durshmeffer. 


\title{
NAUTILUS AMBIGUUS.
}

\section{DER ZWEYDEUTIGE SCHIFFER.}

\author{
T A B. 9. Fig. d.e.f.
}

$\mathrm{T}$

ESTA fpiralis involuta, fuborbicularis, utrinque convexa, centro fubimpreffa, dorfo fubacute carinato, artizulis $\int_{\text {. }}$ thalamis in extimo anfractu confpicuo tre. decim fubelevatis fpiraliter ftriatis, firiis approximatis parallelis; difepimentis antrorfum fubflexuofe couvexis; plano orali convexo, fubcordato, lobis acutis, pertufo punctis fex, utrinque tribus feriatis, lateri parallelis, xqualiter difantibus, $\delta$ in medio tribus minoribus in triangulo pofitis punctis fignato (an pertufis, non contiat), denique in margine $9-10$ foraminulis acute angulatis predito, qua forfan nua cum modo dictis loco orifi;ii funt, cujus alias nulla funt vefigia.

$D_{\text {ie }}$ SCHALE if fchirr lartig, in fich Selbft gewunden, siemlich kreisrund, anf leyden Seiten gleich ftark gewölbt, im Mittelpnnste aber ein wenig eingediritht; der Rücken etwas Scharf gehielet; die Glieder oder Kammern find an der äu. serffen fistharen Windung dreyzehn, etwas erhöhet, nash den Lauf der Windungen geftreift, die Streifen nahe an einander und parallel; die Scheidewände vorwarts etwas gefthlaingelt convex; fo auch die Mïndungsfläche gewälbt, siemllich herafurmig, da die beyden Lappen

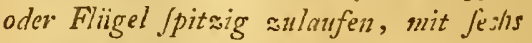
Löshern verfehen, wovon anf jeder Seite drey mit folcher parallel gereihet find und gleich weit von einander abfehen, aush in der Mitte noch drey kleine Eindricke fish aeigen (ob dieje wirnlich die Schale auch durchbohren, kann nicht gewiss behanptet werden). Endlich beobachtet man wosh an Rande 9 bis 10 Jpitswinkelige Lüsher, welche vielleicht nebf den cufgenanniten ainfatt der Mündung 
dienen, von wolcher nan wufsey den keio ne Spur findet.

COLOR albus. cochleis majoribus contenta, a Dno. Spengler.

MENSURA $\frac{1}{2}$ lin. diam.
PATRIA finus arabicus, ex arena in

- FARBE weifs.

VATERLAND der arabifiche Meerbufen, woher fie aus den in gröfsern Scíneken enthaltenen Sande Hr. Spengler erhalten hat.

GRÖSSE $\frac{1}{2}$ Linie in Durchmeffer.

Nota. Forma tefix ambigna. An forte lituus abbaticus (Naut. Semilituus Lim.) pullus? pars nimirum fpiralis absque parte recta? faltem fiructura multum convenit cum illo. Si vero non ifie, fed vere propria hujus generis fpecies eflet, de- Areckten Theil? wenigftens komut ithr uno approximationis generum feu tranfi- Bau viel mit jenem überein. Gefetist tionis ab uno ad alterum eflet exem- aber, er waire es nicht, fondern diefs plum. wirklich eine diefem Gefchlechte eigene Art, fo bätten wir hier abermahl ein Beyjpiel der Amäherung der Gefinlech. ter, oder des Übergangs ron einem aum ander\%. 


\section{NAUTILUS FARCTUS. \\ DER AUSGESTOPFTE SCHIFFER. \\ T A B. 9 Fig. g. h. i.}

TESTA fpiralis iuvoluta finiftrorfa, fubrotundata, ultimo folum articulo maximo, prominente, fupra valde elevata, infra fubplanata, utrinque fubumbilicata, feu medio impreffa, undique punctis elevatis, five granulis minimis denfe fparfis, fcabra; anfractibus finiftrorfis; articulis feu thalanis in extimo folum confpicuo anfractu non nifi octo torulofis, quo facto tefix ambitus repandus apparet; diffepinnentis antrorfum leniter conrexis. De orifivio in duobus fpeciminibus folum adhucdum nobis obviis detrito nihil dici poteft, tam diu, quam non inveniatur unum alterumve integrum.

COLOK albo-flavefcens.

PATRIA Coroncina prope Senam urbem in Hetruria (fofilis).

MENSURA $\frac{2}{3}$ lin. diam,

Die SCHALE ift folnirkelfurmig in fiuh felbft, aber links gerwunden, sienulich rund, nur das letate gröfste Giied ftehet hervor, oben ftark erhaben, unten faft fach, auf beyden Seiten etwas genabelt, oder in der Mitte vertieft, durchaus son dicht ans. geftrenten erhabenen Puncten, oder feinen Körnshen rauh; die Wiudungen lanfenz links; die Glieder oder Kammeru, derenz man in dev. blofs änfsern fishtbaren W'indung nur asht wïhlet, find ftark ge. vojlbt, uiad verurfathen dadur.h, dafs der Unifang der Schale ausgef:hweifter fineint; die Scheidewände find vorwarts gaits forwash erhuber. Vou der Mündung, die in dea beyden voin wins bisher vorgefundenen Bey/pielen abgerieben ift, lafst fich nichts frgen, fo lang bis nicht ein oder anderes unverletates Stiich angetroffen wird. FARBE weifs-gelblich.

$V A T E R L A N D$ Coroncina bey Siena in Tos:anij:hen.

GRÖSSE ₹ Linie im Durchmefler. 


\title{
NAUTILUS SINUATUS.
}

\section{DER BUCHTIGE SHIFFER,}

\author{
T A B. 10. Fig. a. b. c. d.
}

$\mathrm{T}$

ESTA fpiralis involuta, fubflexuora, mediocriter eleváta; ambitu fubovali-repando rel potius fubfunuato, dorfo acute carinato, margine tenui, angufto, acuto; umlilico a latere inferiori impreffo, fupra magno fubprominente punctato, punctis minutis, denfis, elevatis; anfractibus $/ f$ niftror/s, articulis feu thalamis elevatis lavibus, undecim in anfractu extimo confpicuis; difepinentis antrorfum convexis; plano orali nullo; orificio fublineari (fig. $b . d .0$. ) in latere inferiore ultimi articuli convexiore a centro ad peripheriam \& hinc ad latus fuperius planius aliquantum exteufo (latera enim hujus articuli inaquales funt inter fe).

Die SCHALE ift fchnirkelförmig, eirsgevollt, etwas bogig, mittelmäfsig erhaben, mit einem diinnen fohmalen und Scharfon Soume; fie hat auf beyden Seio ten einen Nabel, der ouf der untern Seio te ift vertieft, der oleve aber grofs, etwas erliohet und mit kleinen dicht beyfommen fechunien evhabenen Puncten be Jetat; die Windungen laufer links, die Glieder oder Kammern find erhaben and glatt, wovoin auf der ciufsern Windung I I fichtbar find; die Scheidewände find rovwairts gersölbt; hier befindet fich keine Mündungsfläche; die Mündung Jelbft ift beynahe ftrichförmig ( $f g$. b. d. o.) am Ende der untern eshabenen Seite des let:zten Gliedes, und siehet $\sqrt{2}$ :h vom Mittelpuncte grgen den Umkreis und von da ein wentig auf die obere fichere Seite desfelben Gliedes hinïber (denn die Seiten diefes Gliedes find ungleich unter einander). 
COLOR dilute fufcefcens, a terra ferruginea intus contenta, naturalis fine du. bio albus.

PATRIA regio ad S. Quiricum in agro fenenfi Hetruriæ.

MENSURA $\frac{2}{3}$ lin, diam.
FARBE hell bräunlich von der darin enthaltenen eifcnocherigen Erde; die natiirliche ohne Zweifel weifs.

$V A T E R L A N D$ die Gegend von $S$. Quirico in Sienefifchen in Toscana. GRÖSSE 흔 einer Linie in: Durchm.

\section{NAUTILUS MACELLUS.}

\section{DER MAGERE SCHIFFER.}

VARIETAS $\propto$

ERSTE ABÄNDERUNG

T A B. 10. Fig. e. f. $g$.

TESTA fpiralis involuta fubovali-ro- $D_{i e}$ SCHALE iffpiralartig eingerollt, tundata, utrinque compreffa, exumbili- einigernafsen oval rundlich, auf beycata; dorfo acute carinato; fuperficie ra- den Seiten zufammen gedriickt, ungediatim ftriata, ftriis $f$. coftulis 23 in ex- sabelt; der Rücken ift fcharf kielförmig; timo anfractu confpicuis, fulcis interme- die Oberfäche frahlartig geftreift, won diis lineolis incifis, brevibus, fubparal- den Streifen oder kleinen Ribben feht lelis, trausverfalibus $\mathrm{f}$. fere fecundum man 24 an der äufserften Windung, die ductum anfractuum ftriatis; thalamis \& darwwifchen liegenden Furchen find mit ifthnis antrorfum valde convexis; anbi. kleinen kurzen eingefchnittenen faft patw hinc inde fubinterrupto quaf repan- rallelen Linien in die Quere oder beynahe do; plano orali elevato fubtriangulari nach dem Lauf der. Windungen geftreifi. elongato, bafi fubparabolice incurvata Die Kammern und Scheidewäude find pertufa quinque punctis fubtilifimis, ocu- vorwärts ftark gewölbt; der Unıkreis ift 
lo licet bene armato vix perceptibilibus, hier und da etwas unterbrochen, gleich. \& quidem per medium in linea recta ab fam ausgefchweift; die Mündungsfläche angulo interiori verfus exteriorem, in- ift erhaben, siemtich verlaingert dreyeckig, fuperque prædito ad angulum exteriorem nit einer faft parabolifch eingekriimnten foraminulo paulo majore. Orificium nul- Bafis; längs nach der Mitte hindurch in lum vifibile, ni forte illa quinquie forami- gerader Linie von innern gegen den äunula per medium \& ifisd ad angulum fsern Winkel befinden fich fiinf äufserft ejus loco funt, uti jam in aliis fpeciebus feine, aush mit einem guten Vergrößsefufpicati fumus, \& forfan etiam modo rungsglas kaum bemerkbare durchbroche. dictum foraminulum angulare fumul fi- ne Puncte oder Löchlein, und aufser diephoni pro apertura eft,

COLOR albefcens.

fem noch am äufsern Winkel ein etwas gröfseres. Von einer Mündung ift nichts au Sehen, wenn nicht etwa jene fünf klei se Löcher durch die Mitte und das ans Winkel liegende gröfsere derfelben Stelle vertreten, wie wir bereits an andern Arten rermuthet haben; und vielleishs dienet auch gedachtes gröfsere augleich den Sipho au feiuer Öffuning.

FARBE weifslich.

PATRIA Concretiones Zoophytica maris mediterr.

MENSURA : lin. diam. long.

$V A T E R L A N D$ soopliytifiche Consre. tionen des mittellandifchen Meeres.

GRÖSSE : Linien grösserer Durch. meffer.

Nota. Hæc fpecies admodum appropinculat Nautilo crifpo, ita ut pro fola ejus varietate haberi fere pofet; fed differt præfertim forma valde comprefla, non orbiculari, neutiquam declivi, \& nullo umbilico, nec depreffo, nec prominente. Nomen ei dedimus in relatione ad Naut, crifpum, qui multo crallior eft.

Anmerk. Diefe Art nähert fach fehr furk dem kraufen Schiffer fo, dafs mais fie blofs firr eine Abcinderung defjelben halten hünnte; fie unterfoheidet fich aber von ihm hauptfichlich durch ihre fehr wufammen gedviichte Geftalt, dadurch, dafs fie nicht kreisrund ift, auch nichts weniger als jihe in der Dicke abfällt, und keinen, weder vertieften noch hervorftehenden, Nabel hat. Den Nahnen haven 
suir ihr gegeben in Vergleichung mit dem kraufen Schiffer, der weit dicner ift.

\section{T A B. 10, Fig, h. $i_{0} k$.}

Differt a præcedente rarietate eo, quod tefta fit ventricofior, ftriæ panciores (hic folummodo feptemdecim in anfractu extimo confpicuo); planum orale brevius, nullis foraminulis per medium feriatis confpicuis pertufum, nifi ultimo ad angulum exteriorem fito (forfan cetera propter magnam eorum pufillitatem oculis aufugiunt). De reliquo hac varietas ftructura ventricofiore adhuc magis accedit ad Nautilum crifpum, quam pracedens, \& quafi media inter hunc \& priorem varietatem elt; fed ab co pra. fertim umbilico nullo, cum ommes firiæ in uno puncto convergant, $\&$ ambitu repandiori difcedit, lhisque duobus criteriis magis cum antecedente varietate conyenit.

COLOR \& PATRIA ut fupra.

MENSURA $\div$ lin. diam.
Diefe weicht von der vorigen dadurch $a b$, dafs die S:lhale banchiger ift, die Streifen weniger, (hier blofs fiebzehn an der änfserften Windung) find, die Miündungsflishe kirraer, and mit keinen kleinen ïler die Mitte gereiheten, wenigftens nisht fishtbaren Löshern, das letste an den äufsern Winkel ausgenomnzaen, verfehen ift (vielleisht entziehen fach "die anderna wegen inrer Kleinheit den Gefichte). Ubrigens riliht diefe Aländerung durith ihren anfgetriebeneris Bau sun kranfen Soliffer nowh nither als die vorherige, und masht gleichfan won ihm den Übergang wul erferer; fie unterfcheidet fich aber von illm hauptfaichlich durch dre Abrwejenheit des Nabels, inden alle Streifen in einen

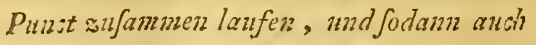
durch den ausgefortweiftern Unkreis, duroh welche beyde Kennseichen fie mehr mit der vorhergehenden Varietat iiberein konmt.

FARBE und VATERLAND wie oberz.

GRÖSSE $\div$ Linie in Durchmeffer. 


\section{NAUTILUS CALCAR。}

\section{DER SPORN SCHIFFER}

T A B. 11, Fig. a. -k. T A B. 12. Fig. a. $-k$. T A B. 13. Fig. a. -1.

Linn. Syft. Nat, edit. XII. p. $1162, \int_{p} .274$,

- XIII. Gmel. p. 3370. Sp. 2.

Schröter Einleit. Conch. Kemtn. I. Bd. p. 9. II.

Schreibers Conch. Ketsmtn. I. Bd.p. 2. II.

Forma hujus fpeciei ita comparata eft cjusque varietates tam multiplices, ut fere impolibile videatur, characterem specificum erui pofle, quo hæc fpecies a reliquis congeneribus difingueretur \& tamen nulla varietas exclucieretur. Pracetcris, ni fallimur, pró vera differentia fpecifica allumere polfumus hanc, quod tefia hujus Nautili a centro tumido verfus marginem acute carinatum valde declivis fit, etfi confitendum, nofiram fpeciem hac ftructura ad Nautilum crifpum valde appropinquare, qui vero aliis criteriis ex. gr. thalamis anguftioribus itaque pluribus $\&$ alio modo flexis \&c. fatis differt. Hæc itaque major diffepimentorum diftantia pauciorque numerus alterum criterium eft, quo fpecies noftra a reliquis diftinguitur. Tertium criterium

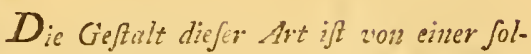
chen Eigenfohraft und ihre Abänderungen fo vielfach, dafs es faft unmöglich s:heint, ein Jpeciffches Kenunzeichen heraus au bringen, wodurch fie ron ithen ubrigen $G e$ fwhesits serrecindten unterfotheden und doch keine Varietat ansgefchlofjen wiirde. Am erften möshte diefs noch eine Jpeciffs:he Differenzs abgeben kömnen, darss die Schale diefes Schiffers ronu hoch erhabenen Mittelpruict gregen den foharf gelielten Rand hin fehr juhe abninumt. Gleichwohl muls man geftehen, days diefe Ait fich hierdurch dent kranfen Schiffer ftark nihert, der hingegen durth andere Merhnualle s. B. durch engere and folglich mehrere und anders gebogene Kammern u. f. f. fich hinlanglich unterfcheidet. Diefe grujssere Evitfernung der Soheidewande von einander wad 
in eo confiftit, quod tefia utrinque mam. ihre geringere Ansahl ift alfo das zweyte milla centrali five umbilico magno pro- Merkmahl, wodurch unfere Art von anminulo, fed planiusculo, prædita fit. De- dern abgeht. Ihr drittes Merhmahl benique quarto difcedit a plurimis aliis ori- flehet darin, dafs fie auf beyden Seiten ficio parvo rotundo fubftellari feu ver- in der Mitte mit einer gro/sen runden rucæformi. Interea non plane omnes va- Warze oder einem etwas hervorragenrietates latera habent valde elevata, va- den, aber wiemlich fachen, Nabel verfehen rietas enim fecunda compreflior eft cete- ift. Endlich zum vierten unterfolieidet fie ris; neque umbilicus femper integer \& fich von den meiften andern durch eine hleilævis, cum in varietate $\delta \&$, loco ne runde faft ftern-oder warzenformige umbilici adfint granula, \& invar.x. umbilicus plane abfit.

Miindung. Inzrvifchen haben nicht alle Abänderungen ftark erhabene Seiten, indem die zuveyte sufammen gedriickter ift, als die ulurigen; auch der Nabel ift nicht alle eit ganz und glatt, inden bey der

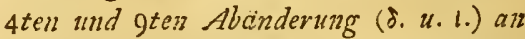
cier Stelle des Nabels mehrere Körner fich befinden und bey der zehnten (x.) der $\mathrm{Na}$. bel gar fehlet.

Ad evitandam omnem, qua exinde

Einer allenfalls hieraus au beforgenden exoriri pofet, ambiguitatem \& confufionem, optimum nobis vifum eft, ex multifariis varietatibus præcipuas eligi, carumque unam, fecundum quam Linnæus fine dubio nomen fuum compofuerit, quoad omnes proprietates primum defcribi, atque reliquas folummodo eatenus afferri, quatenus qualibet a ceteris discedat.

Undentlichkeit und Verwirnung vorsubeugen, ift für das Rathjamfte befunden worden, unter den vielerley Varietaten die v'oraüglichften heraus su heben, eine davon, naih welcher Linné olne Zweifel feinen Nahmen gebildet haben mag, nach ihren Eigenf-hraften an befohreiben, und die iibrigen nach einander nur in fo fern ansufilhen, in wie fern fich jede.von der oudern unterfoheidet. 


\section{T A B. 11. Fig. n. b. c.}

TESTA fpiralis involuta, orbicularis, a centro tumido verfus marginem feu dorfum acute carinatum valde declivis; anfractibus duobus lævibus; ifthmis paucis, in extimo anfractu feptem confpicuis, antrorfum mediocriter convexis, extus colore coerulefcente transparentibus leviterque elevatis; mammilla centrali feu umbilico utrinque magno lævi prominulo fatis depreflo; dorfo circumdato margine membranaceo lato fpinofo, fpinis novem compreflis, partim longis fubaequaliter diftantibus; plano orali fubtriangulari, cruribus introrfum parum incurvatis, bali in angulum valde introrfum retracta; orificio in angulo externo ejus plani, parvo rotuudo fubftellari $f$. verrucæformi, lineolis incifis radiantibus.

COLOK naturalis albus $f$, margaritaceus, in folfilibus vero a terra ferruginea rubida, qua farctæ funt teftre, transparente, vel eas extus inquinante, aut dilute, aut obfcurius cupreus.
Die SCHALE ift fchnirkelförnig eingerollt, kreisrund, und fällt von dens ftark erhabenen Mittelpuncte gegen den Scharfwinkelig gekielten Rand oder Ribcken fohr juhlie ab; hat zwey glatte Windungen, wenig Sclieidewäude, in der äusern Windung nur febeni, fichtba. $r e$, die vorwärts mittelmäßsig gewölbt, auswendig etwas erhaben find und blänlich durchfsheinen; die Beule oder der $\mathrm{Na}$. bel auf beyden Seiten ift groß, glatt, etwas heraus ragend und fark platt gedriickt; der Rücken ift mit einem hautdiunen, breiten und fasheligen Rande ungeben; die nenn Stathel find wufam. mengedrückt, sum Theil lang, zienulioh gleichweit von einaider entfernt; die Mïndungsfäclie mauth faft ein Dreyeck, defen Schenkel ein wenig einwairts gehrimunt find, und die Bafis in einen Winkelffark eimwärts geagon; die Mündung ift in äuscin Winkel der Mündungsfïbine, Wlein, rund, etwas flern-oder warwonförmig, mit eingefinitit enen ftrahligen kleinen Linzien.

FARBE der natililichen weißs oder. perlfarl, bey den folfilen aber bald heller, bald dunkler kupferbrainu, von der röthlichen eifenfshiiffgen Erde, wonnit die Sihalen angefiillet find, die durch folche durohforinet, oder aussen an ihnen klebet. 
PATRIA mare adriaticum, præfertim in littore ariminenf, fofilium Coroncina in terra fenenf Hetruriæ. Hujus varietatis fpecimina follilia quidem a Dno. Abbate Soldani in fedimento nobis mil$\mathrm{fa}$, at neque in ejus Saggio crittografico, neque in Teftaceographia reprælentata funt fpinofa, fed folummodo dentata, \& fequentis varietatis margine in. tegro prædita.

MENSURA $\frac{x}{2}-2 \frac{x}{2}$ lin. dian.

VARIETAS $\beta$.
$V A T E R L A N D$ das adriatifche Meer, vorsitglich am riminifsien Geftade; der fofflen Coroncina bey Siena im Toscani. fchen. Von diefer Varietät find swar von Hrn. Abt Soldani einige Stiiske in Sedinent uns iiberfchickt, allein weder in feinen Saggio orittografico, noch in der Teftaceographia abgebildet worden, fondern blo/s die gezihnelte und die von der folgenden Abanderung mit ganwem Rande.

GRÖSSE $\frac{1}{\frac{1}{2}}$ Linie im Durchmefer.

\section{ZWEYTE ABÄNDERUNG.}

\section{T A B. I I, Fig. 'd. e. $f$.}

Jan. Planc, Conch. edit. 2. p. 85. tab. 1. fig. 12. S. T. V. छfig. 13.z. Z. (rudes). Martini Concb. Cab. Bd. 1. tab. 20. fg. 180. 181. (rud. ex Planco).

Soldani Saggio orittogr. tab. 1. fig. g. G.

\section{Teftaceogr. T. I, P. I. tal. 33. fig. B.}

TFSTA lævis, compreffior ceteris, ifthmis \& umbilico prominulo lævibus, margine membranaceo integerrimo.

COLOR, PATRIA \& MENSURA ut in variet. præced. quandoque adhuc majoris molis.
Die SCHALE ift glatt, nehr $\approx u$ fam. men gedriickt, als die ïbrigen, die Scheidewände und der ein wenig vorragende Nabel find auch glatt, der Rand diinnblïtterig und gans.

FARBE, VATERLAND u.GRÖS$S E$ wie bey der erften Varietät, manchnahl woch grüjser. 


\section{T A B. 11. Fig. $g . h_{0}$}

TESTA Jeris, ifilmis granulatis, umbilico prominulo levi, margine membranaceo fpinofo, fpinis feptem, fed brevioribus, quam in varict, $\alpha$.

COLOR \& PATRIA, ut fupra, fed tantæ magnitudinis noudum hujus varietatis obvenerunt fpecimina, quam in prexcedcutiizus.

VARIETAS 8.
Die SCHALE ift glatt, die Scheidewände find mit Kömern befet at ; der etwas hercorragende Nabel glatt, der Sanm dinnaliatterig und fachelig, mit fieben Stacheln, die aber liirser find, als in der erften Abcinderung.

FARBE and VATERLAND wie ol en, die GRÖSSE aler ift bey diefer $V a-$ rietcit noch nicht fo mefhalich vorgenomanen, wie ley den vorigen.

VIERTE ABÄNDERLNG.

T A B. 11 , Fig. $i . k$.

TESTA lævis, ifhmis \& umbilico graDie SCHALE if glatt, die Scheidenulatis, margine membranaceo dentato, wände und der Nabel find gelurnt, der dentitus octo planatis fubrequaliter lon- Sonm diumblatterig und geashuelt, die ge diftântibus.

COI.OP, PATRIA \& MENSURA ut proxime pracedens, achi Zihne find pint, weit, aler etwas ungleich von einander alfethend.

FAREE, VATERLAND U. GRÖS$S E$ wie waihft vorher gehende. 


\section{T A B. 12, Fig. a. b. G.}

Soldani Saggio orittogr. p. 9\&. tab. I. f.g. 6. 7.

- Teftactogr, T. I, P. I. tab. 59. fg. qq. rr.

hac differentia, quod harum figurarum margo dentatus; noftri exemplaris vero fpinofus lit. mit dem UnterfCbied, dafs an diefen Figuren der Rand gezäbut, an unferm Beyfpicle aber fara cliclig ift.
TESTA denfe minutim granulata, excepto ultimo articulo feu thalamo, hoc ifthmisque $\mathcal{E}$ umbilico levibus, margine membranaceo fpinofo $\mathcal{E}$ partim fubtiliter crenato, fpinis octo æqualiter diftantibus acutis, duabus valde longis.

COLOR, PATRIA \& MENSURA ut fupra.
Die SCHALE if wit jehr kleirzen Körnern dicht befetst, das letste Glied oder Kammer ausgenommen, diefes, die Sicheidervainde und der Nabel find glatt, der hüutig dïnue Saum ift ftachelig und zum Theil fein generbt, die acht Staihel find gleichreit von einander entfernt, Spitzig, zwey dazon rehr lang.

FARBE, VATERLAND u. GRÖS. $S E$ wie oben.
Nota. Hæc rarictas non tam propter teftam granulatam, quæ copiofius invenitur, quam pracipue ob fpinas fubtiles, attritui perfacile obnoxias, inter rariflimas ponenda.

Huic tabula fub lit. c. adjecta funt duæ icones fpinarum, quarum una Spinam longam acutam cum fragmento marginis membranacei minutim crenati, altera vero breviurem latioremque latere levifime excavato reprefentat.
Anmerk. Diefe Varietät gehört nicht Sorvohl wegen der gekörnten Schale, die fich hïufger findet, als hauptsichlich wegen der feinen Stachel, die fich leicht abftofsen, unter die Jeltenfletz.

Diefer Tufel find swey fehr vergrüfserte Abbildungen won Stacheln bey fig. c. beygefiigt, deren eine einen langen Jpitsigen Stuchel mit einem Bruchfii:k des blätterig dinnen fein geherbten Saumes, die andere aber einen kïracm, breitern, 
auf einer Seite fehr feicht ausgehöhlten Stachel vorflellet.

\section{T $A$ B. 12. Fig. d. e. $f$.}

TESTA, ifthmi \& nmbilicus prominulus laves, margo membranaceus integer, lafis f. pars tefta antcrior ex tribus quatuorve thalamis magis magisque autrorfum coarctatis compofita, ad inftar roftri apice, in quo crificinm fubfiellare, fubnodulari terminati frolongata, finulque in loco, ı:bi alias planmm orale obfervatur, fecundum ejus longitudinem angulis egrellivis regrefivisque $\mathcal{E}$ triangulis elongatis acutis alternantibus obtufe triplicata. Duus. Abbas Soldani, a quo originalia gleba terrex immixta venerunt, fune dubio ejusmodi nautilos in Teftaceographia T. I. P. 1. tab. 53. fig. gg. hh. ii. kk. mm. \& tab. 59. fig. qq., quos pag. 64 Nantilos carizatos, ut \& Lenticulas nuncuparit, iconibus reprefentari volnit. Sed icones iftæa at mon fatis fideliter elaborate, ant nulla nofiris fatis fumilia inventa funt excmplaria ; etenim icones itidem æri incifæ partem rofiratam multo breviorem monfirant, quam in noftris fpeciminibus ea reperitur, infupcrque cadcm minus exactc delinea. ta eff.
Die SCHALE, die Scheidewände und der feisht hervorragende Nabel find giatt, der hiutig diune Sanm ift gans, die Bafis oder der vordere Theil der Schale, der ans drey bis wier fish zorwcirts verengernden Gliedernl leftehet, ift in eine etwas hilotenfurmig sugefpitste Art einer Schnauze (woran die etwas fernförnige Mindung lefindlish iff) verlängcrt, die angleich an dem Ort, wo fonft die Mündungsfache ift, wash ihrer Länge nit ein- und ansffringencen Win.eln, nnd alweshfelnden ffitacn arlangcrten Dreye. chen drey fumpfe Fulten masht. Hr. Abt Soldani, von den aubh die Originale in einer Erdmaffe liegend herriihren, mag dergleichen vermuthlich in Seiner Teftaceographia T. I. P. I. tab. 58. fig. gg. hh. ii. kk, mm. et tab. 59. fig • qq. $200 \mathrm{er}$ fie pog. $\sigma_{4}$ Nautilos carinatos, w:e auch Leuticulas nannte, halen wollen abbilden laffen. Allein entweder find die Abbuldungen wicht getreu genug gemacht, oder er hat keine unjern fo gleich hommende Exemplare gefunden; denn die abgubildeten haben eine weit hïrsere Schnause, als die nujerigen, und iuber dieß ift fe anch K 2 
Hrec varietas etiam inter rariora eft.

COLOR füfcefcens.

P'ATRIA adhucdum nota folum eft Ripalta ad S. Quiricum in agro Senenfi Hetruriæ.

MENSURA I $\frac{x}{4}$ lin. longitudo maxima.

VAFIETAS nicht all:u genan gemacht. Diefe Abänderung ift auch unter den feltenen.

$F A R B E$ briunlich.

$V A T E R L A N D$ ift bisher bekanntlich nur Ripaltabey San Quirico unweit Siena in Toscanifshen.

GRÖSSE $1 \frac{\mathrm{x}}{4}$ Linle größste Lïnge.

\section{T A B. 12, Fig. g. $h$.}

Jan. Planc, Concb. p. 12. tab. I. fg. 3. H. (rudis).

Ledermüller Microfcop. Gem, u. Auz. Ergötz. tah. 8. fig. c. (vud.).

Martini Conch. Cab. I. tab. 19. fig. 171. (ex Ledevmilll.)

TESTA ifthmi \& umbilicus prominulus laves, absque margine membranaceo, mullisque fpinis $\mathbb{S}$ deutibus.

\section{COLOR plurimum albidus.}

PATRIA naturalium mare adriaticum \& mediterraneum, foliliumque Coroucina Senenfis in Hetruria.

MENSURA $\div-1 \div \operatorname{lin}$.

VARIETAS \&.
Die SCHALE, die Scheidewände und der feicht hervorragende Nabel find glatt, hingegen fehlt der diinne Saum, wie auch die Stashel and Zaime.

FARBE meiftens weifstich.

$V A T E R L A N D$ das adviatifche und mittellandifche $\mathrm{Meer}$; der folfilen Coroizcina im Toscanifinen.

GRÖSSE $\frac{1}{2}-\mathrm{I}_{4}^{ \pm}$Linie.

ACHTE ABÄNDERUAG.

\section{T A B. 12, Fig. i. $k$.}

TESTA, ifthri \& mammilla umbili- Die SCHALE, die Scheidewände und calis læves, fine margine membranaceo der Nabel find glats, kein diunzer Saum 
\& fpinis, fed cum fex dentibus compref- noch Stachel, aler fechs breit gedriickte lis fubaqualiter diftantibus, quibus folum sientich gleichweit won einander abftehena pracedenti varietate diftinguitur. de Zähne find vorhanden. Blofs dursch diefe unterfcheidet fich diefe Varietüt von der vorher gehenden.

COLOR, PATRIA \& MENSURA ut pracedentis.

FARBE, VATERLAND u.GRÖS$S E$ wie vorther gehende.

T A B. 13. Fis. a. $b$.

TESTA \& ifthmi granulati, loco umbilici tres papille feu grana majora in triangulo pofita, margo membranaceus fubdentatus (duobus quafi - dentibus).

COLOli flavefcens, granula ifthmorum \& centri cærulefcentia.

PATRLA \& MENSURA ut fupra.

VARIETAS $*$.
Die SCHALE und die Scheidewände find mit kicinen Körnern befetst; anfatet des Nabels fieht man drey Kü̈tchen oder gröfsere Kömer inn Dreyeck beyfanmen, der dinnz Saum ift einige:majen geacihnelt (indem swey Hervorragungen wie Zuhize diranf find).

F ARBE gelbith, die Hörner der Zwishenrecinde und der Aitte find blaulioh. PATERLATDD und GRÖSSE wic oben.

ZEHNTE ABÄNDERUNG.

T A B. 13. Fig. c. d.

TESTA \& ifthmi laves, margo fub- Die SCHALE und die Scheidewände membranaceus eminentiis tribus quatuor- fand glatt, mait benerket nur whn Theil ve planis fubdentiformibus, nulla mam- einen herrorfeherden blattdinnen Saum, 
milla umbilicalis. Hac umbilici abfentia der fich in drey bis vier Zähnen ähnliche hæc varietas ab omnibus reliquis digno. fache Erhalenheiten endiget; ein herzorfcitur.

COLOR albidus.

PATRIA mare mediterraneum.

MENSURA, lin. diam. ragender Naliel aler fehlet ganzlioh. Durch diefe Abweferheit des Trabels un. terfoheidet fich diefe Varietät allein fichou von allen iilvigen.

FARBE weifstich.

$V A T E R L A N D$ das mittclländifone Meer.

GRÖSSE 1 Linie in Durchanefer.

VARIETAS $\lambda$.

EILFTE ABÄNDERUNG.

T A B. I 3. Fig. e.f. $g$.

TESTA, ifthni \& umbilicus prominulus laves, margo membranaceus integer, thalami rari, nimirum in anfractu externo vix quinque \& if hmi folum quotuor confpicui, quod in hoc fpecimine precipue notabile ef, idque delineatione fane dignum reddit, cum in aliis varictatibus plerumque octo novemque thalami, imo nonnumque plures, velut in Varietate $\beta$ duodecim offenduntur. Memorandum adhuc venit, quod, præter omnibus varietatibus commune orificium parvum rotundum \& crenatum, hic difincte in angulo exteriore plani oralis (fig. g. lit. o.) a dicto orificio introrfun vergens vimula ringens $f$. marginata, elongata, ab initio angufta, fenfim dilatata
Die SCHALE, die Scheidewände und der etwas hervorragende Nabel find glatt, der diinullatterige Sal mif gans. Das Sonderbare an diefen Stiisue, und welches daffelle eincr Albildung werth $\approx u$ machen fichen, if , dajs die Kammern in gevinger Ansahl find, inden die ciusere Windung nicht gar fiinf Kommern und nuur vicr Scheidewände seigt, da doch bey andern Vuriaten gewuhnlich 8 bis 9 Kinnmern in gedachter Windung angetroffen werden; ja man findet ihrer manshmahl noch minere, wie oben bey der wweyten Alcinderung awölf $\approx u$ fehen find. Merlwirdig if noch, dafs fich hier dentlich, anfier derbey allen vorigen Ab. änderungen gewöhnitiven engen, runden, 
\& obtufe clanfa obfervatur.

COLOR albidus.

PATRIA Coroncina in Hetruria.

AูENSURA $\stackrel{3}{\sharp} \operatorname{lin}$. gekerbten Mïndung im äußsern Winkel der Miindungsfiche (fig. g. lit. o.) eine son der Mündung eintwiorts gehende lingliche kleine fein gefaumte Spalte zeigt" die Anfongs gans enge beyfanmen ift, fodann nash und nach fich evweitert und endlich augernudet fohließst.

$F A R B E$ weifslich.

$V A T E R L A N D$ Corontcina int Toscanifchen.

GRÖSSE $\frac{s}{4}$ Linie.
TESTA \& iffini leves, unzbilicus prominens pragrandis lævis, cujus diameter fubaqualis eft femidiametro majori totins teftæ, absque margine membranaceo ullisque dentibus, fed cum spinis fex longis fubtilibus in dorfo acuto, quibus \& umbilici magnitudine hre varietas rara reliqutas antecellit.

COLOR albefcens \& fufcefcens.

PATRIA mare mediterraneum \& Coroncina in Hetruria.

MENSURA $\frac{*}{2}-\frac{8}{4}$ lin.
Die SCH ALE und Scheidewände find glatt, der hervorragende Nabel ift auch glatt, und felur grols, indem fein Durchmegfer faft die Hailfte des laingcrn Durchmelfers der ganzeiz Sshale beträgt; der diune flathe Sann aber and die Zilne fehren, hingegen fitwen auf der fcharfen Rii-kenkante feilis lange feine Stachel. Diefe und der grofse NTabel seichnen diefe feltue Varietiat vor andern befondera aus.

$F A R B E$ ueifslich und bräunlich. $V A T E R L A N D$ das mittelländifclie Meer und Coronsina ins Tosianifshen. GRÖSSE: 
Nota 1. Ad reque cognofcendam hujus fpecici fructuram interiorem in eadem tabula i 3 . fub. fig. $k$. l. Nautili cal. caris dimidiati iconem dedimus, ex qu? patê, quomodo circa centrum orbieulare cavum thalami valde ampli (quorum hic 12 numerantur, at duo tresve adhuc propter deftructam parten tefte anteriorem deficere polfunt) per duos anfractus fpirales crefcendo circumvolvantur, quorum fubtiliona fatis obliqua diffepimenta latus convexum antrorfum, i. e. verfus nltimum thalamum five oriticiurn obrertunt.

Nota 2. Varietates hucusque defcriptwe inter rcliquas hujus fpeciei funt precipux. Facilc plures reprafentari potuiffent, fed non nifi fuperflua augmentatio operis $\&$ impenfartum fuifet, quo oncre lector emptorque onmi pofibili modo eximendus eff, prafertim cum jam omnes in prolatis fpeciminibus contentae fint. Dantur ex, gr. exemplaria tefar gramulata non folnm longis fpinis, fed etiam dentibus; margine integro, crenato, angulto, lato, se absque hoc; unbiticolavi, vel sranulato, ctian mullo \&c. In ytalibet Varietate non nif maxime convenientes icones ex fcriptoribus allegatæ funt ideoque variæ prætermiflæ, quæ tamen ad Naut. Calcar pertinent. Hæc funt:
1. Anmerk. Lm anch den imnervi Bau diefer Art au lcunien, ift ouf elen der r.3ten Tafel ley k. und l. ein bis aur Haifte anfgelchliffener Spormantilis vor. geflellet worden. Mnn fiehet win diefer Ab. bildung, wie un den kreisfurmigen hoh. len Mittelpunst die fich immer vorgrö. fiernden siemlich weiten Kannucrn, (diren hier 12 geaühlet acerden, aber noch a bis 3 vegein des ureggebrochenein Vordertheils fehien nögeni) in wwey Sprialwindlungen hernmlaufon, derenfeinere wienulich finef fachende Zwifchenwitude ihre runderhabene oder convexe Seite zorwarts, d. i. gegen die letate Kanmer oder. Minzdung sul, gejehret haver.

2. Anmerk. Die jetat lefhrielenen Alinderungen find die zorsigglishlen unter denen, die manz won aiefir Ait hat. Mon hitte leicht noch mehreve anfiihwen zind abbilden köznan; es wiirde aber nur eine uniüthige Vargröfernng dis Werk.es und der Kofene, die nan dem Lefer and lítufor befstmëglichft erfparen muss, gewefen feyn, da ohnchin fohon alle in den mingelurachten Stiivien enthulten find. So giebt es : B. Exemplare, deren Schale gekurnet if , ferner nicht nur nit langen Stacheln, fondern ansh mit $Z$ ulmcn; nit ganacm, geherbten, finnalen, br eiten Rande, mnd ohne denfellen; mit glattenn und gekörnten Nabel, u..$f$. Bey jeçer $V a$ rietcit uurden die auf das genanufte zu- 


\section{$8 \mathrm{k}$}

treffenden Abbildungen angefiihrt; mit. hin mufsten nothwendig manche weg bleilen, die doch zum Spornfohiffer gehören. Diefe find folgende :

Qualtieri Ind. zeft. sab. 19. fg. B. C. (vud.)

Martini Concb. Cab. B. I. zab. 19. fig. 168. 169. (ex Gualt.)

Soldani Saggio orittogr, tal. 1. fig. H. K. L. O.

- Teflaceogr. T. I. P. I. tal. 3). fig. E. Ub. mu, on.; tab. 34, fig. dd. ce.; tab. 50. fg. cc,; tah. 57. fig. S. Sf. T. Tt. ? tab. 59. fig. ec. ff. gg. bh. ii. kk. $l 1$. mim.; tab. $57 . \mathrm{fg} .55$.

Omnes hæ figuræ certo ad hanc fpe- Alle diefe gehören gañ gewißs au dieciem pertinent, cujus varietates transpo- fer Art, deren Abcinderungen man durch fitione reciproca variarum proprietatum Verfet:ung der verf:liedenen Eigenfshaffacili negotio ad 40 vel plures augeri ten mit geringer Miihe bis anf 40 und polfent. Plurima iftarum admodum fre- höher bringen könnte. Die meiften $V a$ quenter occurrunt, aliquot autem rario- rietciten find siemlich gemein, einige aber res funt, paucæ rariffimæ. feltner anzutreffen, etliche wenige fehr Selten. 


\title{
NAUTILUS PAPILLOSUS. \\ DER VIELWARZIGE SCHIFFER.
}

\author{
T A B. 14, Fig, a. b. c.
}

IESTA fpiralis involuta, fubrotunda$\mathrm{ta}$, utrinque valde convexa, (non declivis), non umbilicata; dorfo acute carinato, margine membranaceo parvo; anfractibus levibus, hinc inde papilla folitaria; diffepimentis antrorfum leviter convexis, extus papillofis, papillis hemifphæricis $\mathcal{S}$ ovalibus inæqualis magnitudinis \& fitus, in medio, loco umbilici, denfe accumulatis. Orificium in hoc nobis adhuc unico fpecimine diffractum eft.

$D_{\text {ie }}$ SCHALE ift fonirlelartig eingerollt, sienzlich angerundet, anf beyden Seiten ftark gewölbt (nicht jaihe ablaufend), ungenabelt ; der Rücken fcharf gehielt, mit einem fchmalen, diinnblatterigen Sanme; die Windungen find glatt, nur hier und da fitst ein einzelnes Warrchen oder Knözihien; die Scheidewände find vorwärts jeicht gervölbt, auswendig mit dergleichen Waivchen bedeckt, die theils halbkugelig, theils oval, von ungleicher Gröfse und Liinge find, wnd in der Mitte anf einen Haufen gedrängt ftatt eines Nabels aufanmen laufen. 'Von der Mündung kann man michts anfiliren, weil folche an diefent unferigen einsigen Beyfpiele weggebrochen ift.

COLOR albo-luteus.

PATKIA mare adriaticum.

$F A R B E$ weifsgelblich.

$Y A T E R L A N D$ das adriatifche Meer.

MENSURA $\frac{\text { I }}{2}$ lin, diam.

Nota. Hec Cochleola maguam cum GRÖSSE: Linie im Durchmelfer.

Anmerk. Diefes Schneckchen hat mit Nautilo Calcari habet fimilitudinem \& den Spornfchiffer cine großse Ähnlivhlieit 
forfan mera varietas ejus efi, quæ $p a$ - und ift vielleicht mur eine blofse Abändepillofa vocari polfit; interea cum fpeci- ming davon, die-man die warzige nenminis nofiri elevatio lateralis regulariter nen lännte; indeffen da die Seitenerhöconvexa $f$. circularis fit, non declivis, hung an inferm Stiilihe regelmafsig rund quod criterium principale Nautili Calca- gewojlbt oder zirhelbogenförnig, und ris eft, iftud pro peculari fpecie adopta- nicht jöhe ablaufend ift, welches letztere vimus. Ceterum id, quod porro tanquam ratio pro fpecie propria militans allegari pofet, quod nimirum nofira umbilico tanquam altero criterio Naut. Calcaris careat, nobis non fufficiens eft, quia etiam varietas iftius Nautili umbilico de. fituta datur. (vid. Tab. 13. fig. c. d.) cin Hauptmerkmahl beym Spornfshiffer abgibt, fo haben wir jenes als eine eigene Ait angenommen. Dasjenige iibrigens, was ferner aun Behif einer eigenen Art angefilht werden künnte, days nehmlich die unfere keinen $N^{\top}$ abel habe, der das andere Kennzeichen des Spornfshiffers ift, finden wir nicht fiir fattfan hinreichend, weil es auch beym Spornjchiffer eine $A b$ änderung gibt, die olne Nabel ift (fiehe Tab. ' 3. fig. c. d.). 


\title{
NAUTILUS SPENGLERI.
}

\section{DER SPENGLERISCHE SCHIFFER.}

\author{
T A B. 14. Fig. d. -i. \& T A B. 15. Fig. a. -k.
}

Linn. S.ft. Nat, XIII. Gmel. p. 3371, Sp. 10.

Spengler Schrift. diin. Gefellfch. Kopenh. I, Th. p. 373. fqq. tab. 2. fg. 9. a.b. G. (afjimil.).

Schröter Einleit. Conch. Kennerı, I. Bd. p. $75^{\text {G. I.) }}$

- - Neuc Litter. ". Beytr. z. Naturg. I. Bd. p. 309. tab. 1. fig. 3 - 6 . (ajjimili.).

Schreibers Cench. Kenutu. I. Bd.p. 5. n. 10.

VARIETAS $\propto$.

ERSTE ABÄNDERUNG.

T A B. 14. Fig. d. e. f.

TESTA fpiralis involuta, fubrhombæa, $D_{\text {ie }}$ SCHALE ift fchnirkelartig eingeutrinque valde convexa, tota fcabra rollt, beynahe rautenformig, auf beyden (uti bene D. Schrötcr; non lævi, ut DD. Seiten ftark gewölbt, durchaus rauh (wie Spengler \& Gmelin.), fubftantia enim ex- Hr. Schröter gut angemerkt hat, nicht tus minus compacta, ex granulis mini- glatt, wie Hr. Spengler von den feinimis compolita, permultisque majoribus gen angegeben und $\mathrm{Hr}$. Gnelin es ihm f. verruculis irregularis formæ obtecta, nachgefolirieben hat), ixden die Subfranz \& (quod maxime fingulare in hac Specie) anjen lockerer als innen, aus Jehr kleinen dorfo obtufo prædito protuberantiis tri. Körueru zufammen gefetat und mit fehr 
bus elongatis fubteretibus, longitudina- vielen größern oder Wärachen von unliter leviter ftriatis, minutim granulofis, regelmäfsiger Geftalt überdeckt ift; anch aculeorum inftar, apice obtufo, fingulo (was diefe Avt auf das Sonderbarfe ausex quolibet trium angulorum; quorum seichnet) fehen aufrecht auf dem Aumpf medius in apice \& medio valde verrucu- zugernudeten Rüclien drey lang ausgelofus vel potins ex coacervatis granulis freckte zientichrunde, oben abgeftumpftuberofus, ant duplicato-clavatus eft, cui te, nach der Länge feicht geftreifte, mit cx oppofito parva apparet protuberantia feinen Körnshen befetate Hervorraguntanquambafis vel primordium quartiacu- gen gleich Stacheln, deren jeder anf eilei, aliaque parvula ad orificium; an- nemt von dreyen Ecken fitst, woz'on der fractibus dextrorfis tribus vel quatuor (vid. icon teftæ dimidiatæe infra tab. I5. fig. i.k.), thalamis omnibus occultis; difSepimentis antrorfum leviter convexis (vid. ibid.); orificio fubovali vix promi. nente.

mittleve Stachel an der Spitae und in der Mitte mit vielen Wirrschen ungeben, oder vielnuehr aus sufanmen gehiuiften Körnern häckerig oder doppelt heulcnfürming ift, welchem gegeniiber eine fchrovche Erhabenheit gleich dem Grundanfat:e oder Anfang des :ierten Stachels fich befindet, und nosh eine Kleine neben der Miindning. Die drey bis vier. Wiudningen luafen rechts (Siehe die Abbildung einer bis aur Hilfte oufgejchliffenen Schale unten tal. 15 fig. i K); die Kammern fnd alle verborgen; die Scheidewände vorwärts feicht gewölbt ( $S$. ebend.); die Mündming if ein wenig oval, kawn merklich hervorragend.

COLOR albo-flavefcens.

PATRIA ex arena, qua Buccinum calficleum Gmel. maris Indire orientalis, præfertim finus arabici repletum erat, a D. Spenglero depromptum fpecimen $\&$ nobis miflum.

MENSURA 1 lin. diam. cum aculeis.
$F A R B E$ veifsgeiblich.

$V A T E R L A N D$ aus dem Sande, wonit ein Bucinum caffideun Gmel. des oftind? shen Meeres, infonderheit des arabifshen Meerbufens, angefiillet war, von Hru. Spengler hervorgefucht und hieher iiberf-hickt.

GRÖSSE I Linie im Durchm. Sanmut den Stavheln. 
Nota. Dnus Gmelin hanc fpeciem una cum Naut, unguiculato (Syft. Nat. XilI. p. 3372. fp. 11.) mox poft Nautilum Spirulam Lipn. (p. 3371. fp.9.) inter eos Nautilos linnæanos fpirales rotundatos pofuit, qui anfractus habent disjunctos. De ratione, cur hoc fecit, nobis plane nou liquet, cum utramque fpeciem, nimirum Iomam \& I I mam anfractibus contiguis gaudere evidenter pateat. Qua de re hoc potins errorem tranferiptoris vel typographiæ efle credere malumus.

VARIETAS $\beta$.
Anmerk. Herr Gmelin fetzte diefe Art nebft den Nant. unguiculato ( $\varsigma_{y} f t . N$. XII1. p. $3.37^{2}$ (p. 11.) gleich nach dem Naut. Spirula Limn. (p. 337 I.Sp. q.) unter diejenigen linneifshen fpiralvund gewundenen Nanitzlos, die abftehende Windungen haben. Warum er diefes gethan habe, davon Jehen wir gav heinen Grund ein, da offenbar beyde Arten, nehnlich die rote und I ite anffehende Windungen haben. Wir wollen alfo lieber glauben, daf dießs ein Verftofs des $A b$ fchreilers oder der Drucherey Jey.

\section{T A B. 14. Fig. g. h. i.}

Differt a priori varietate forma fuborbiculari \& compreffiore, granulis minoribus, protuberantiis marginalibus $f_{0}$ aculeis quinque brevioribus, quorum quatuor in altera femiperipheria fubæqualiter diftantes, in altera vero quintus folitarius illis quatuor oppofitus. Ceterum, uti ex fitu orificii fubrotundi elucet, anzfractilus dextrorfis convenit cum prima varietate.

COLOR, PATRIA \& MENSURA Mt fupra.

\section{ZWEYTE ABÄNDERUNG.}




\section{VARIETAS $\gamma$. DRITTE ABÄNDERUNG.}

\section{T A B. 15. Fig. ส. b. . .}

Difcedit a præcedentibus forma fubquadrangulari \& magis comprefla, atlfractibus contrariis f. finiftrorfis, dorfoque magis carinato \& protuberantiis fubteretibus f.aculeis quatuor brevioribus.

COLOR, PATRIA \& MENSURA ut in prioribus.
Weicht zon den zuey zorhergehenden at durch die faft viereckige wnd noch melv alyanmen gedriickte Geftalt, durih die verkehrt oder links lanfende Windungen, den mehr gekielten Rïcken, und die wier kiirzere sienilich runde Hervorragungen oder Stachel.

FARBE, VATERLAND u.GRÖS$S E$ wie worher gehende.

\section{VIERTE ABÄNDERUNG. \\ VIERTE ABANDERUNG.}

VARIETAS $\delta$.

$$
\text { T A B. 15. Fig. d. e. f. }
$$

Differentia inter hanc primamque \& tertiam in eo confiftit, quod fit fuborbicularis, uti fecunda; ab omuibus autem co differt, quod in ambitu fubcarinato tredecim protuberantiis feu quafi - dentibus \& quidem feptem elevatioribus fubconicis, quatuorque minus prominentibus, fed latioribus $\&$ duabus parvis eminentiis, fed omuibus irregulariter a fe invicem diftantibus predita fit. A varietate prima vero hæc fpeciatim difcedit granulis folum minimis. Ceterum conve-
Der Unter/chied wwifchen diefer, der erften und dritten beruhet daranf, da/s fie zientich kreisnund ift, wie die wweyte; con allen aber geht fie dadurch ab, darfs fie auf dew etwas gekielten Rinnde mit dreyzelm Hervorragungen oder gleichfan Zahnen verfehen ift, wornmter fieben etwas kegelfürnige längere, vier kürsere, aber breitere, und awey wenig merkliche Erhabenheiten fich befinden, die alle unregelniifsig von einander abfehen. $V$ on der erften Varietät aber weicht fie 
nit cum varietate tertia anfractibus fun- lefonders durch die kleinen Körnshen $a b$. Arorfis.

In iibrigen aber homnt fie durch die links laufenden Windungen mit der drit. ten iilerein.

COLOR \& PATRIA ut fupra. MENSURA $:$ lin. diam.

FARBE n. VATERLAND wie oben. GRÖSSE: Lin. im Durchm.

VARIETAS $\varepsilon$.

- FÜNFTE ABÄNDERUNG.

T A B. 15 . Fig. g. h.

Variat ab omnibus præcedentibus in

Geht von allen worher gehenden darin eo, quod neque dentata, neque aculeata fit; de reliquo convenit cum varietate prima granulis majoribus $f$. verruculis $\&$ lateribus ventricofioribus; cum eadem vero varietate $\&$ fecunda anfractibus $d e x$ trorfis; \& cum dicta fecunda aliquantum forma fubrhomboidali, f. fubquadrangulari, fed angulis valde rotundatis. al, days fie weder Zihne, nosh hegelformige Stachel oder Dürner hurt; iibrigens finunt fie durch grö́sere Kürner oder Wairschen und durch bauchigere Seiten mit der erften Abcindernng iiberein ; mit eben diefer aber, und der sweyten durch die rechts laufenden Windungen; und mit gedachter aweyten einigermafsen durch den etwas rautenformigen oder wiemlich vierevkigen Bau, wo aber die Ecien ftark zugerundet find.

COLOR \& PATRIA ut fupra.

MENSURA $\frac{3}{4}$ diam.

FARBE nnd VATERLAND wie vorige.

GRÖSSE : Linzie im Durchm.

Nota. 1. Adluuc aliam adferre potuinemus varietatem iconi in Dni. Schröteri cit. fig. 5. reprefentatæ fatis affimilantem, fed fpatio parcendi gratia eam omi-

1. Anmerk. Wir lönnten snoch eine andere Abänderung anfilhren, die nut der in Hrin. Schröters angefïhrten sten Figur vorgeftellten Abbildung zienliche 
fimus, loco ejus autem iconem fpecimi- Ähnlichleit hat ; allein su Erfparang des nis hujus fpeciei fecundum ejus latitudi- Raums haben wir fre weggelaffen, an denem (\$ lin. diam. cum aculeis) dimidia- ren Statt aber die Abbildung eines nach ti in der Breite (s Linl. im Durchm. wit deis Stachelnz) bis aur Hilfte angelifhiffenen Stiickes diefer. Art ouf der

T A B. 15. Fig. i. $k$ :

dcuimus, ex qua patet, tefam ex tri- leysebracht, worous erhellet, dus die bus quatuorve anfractibus, centro glo- Schule aus drey lis vier Windungen bebulari folido, compoftan, in relaticne ftehet, die einen fugeligen unsgefiillten ad magnitudinem fatis craflam effe, ifth- cder ganaczi Mitzelpunct haben, und welmosque quadraginta, \& quod excurrit, che in Vurhiltnifs su ihrer Gröjse ziemantrorfum convexos, quos vero in cit. lich divi iff; dars ferner die etlich und icone Spengleriana fig. c. contrario mo- viersig Scheidewcinde zorwärts gewölbt do, fcil. retrorfum convexos f. antror- find, die alier in der angefiihrten Speng. fum concavos vidinus, furfan ex incuria lerifhen Figur c. umgeucnilet, nehmlich pictoris vel fculptoris, quod in objectis tam minutis facile erenire poleft. Porro ex hac teftre fectione videri licet, protuberantias five longiores, five breviores ton inanes feu tubulofas, fed totas folidas efie. Denique quoad patriam adhuc aduotandum venit, fpecimina a Dno. Schröter allegata non ex finu arabico, fed ex fpongia maris adriatici deprompta effe. als zorwärts hohl algebildet fich seigen, viclleicht aus an geringer. Sichtjanheit des Mihlers oder Kupferflechers, walches fich bey fo kleinen Gegenftinden leicht ereigmen hann. Ferner ift ans diefen Schalenanf shnitt au erfehen, dafs die fowohl langern als kïrsern Herworragumgen nicht hohl oder rührig, fondern gans dicht find. Eadlich kommt auch leym Vaterland noch zu lemerhen, dafs die von Hrn. Sicho. ter angefilhrten Stiishe nicht in arabi. fchen Meerlusen, fondern in einer Spongie des adriatifon Meeres gefunden wore den find. 
Nota 2. Præter hæc in ftatu fuo naturali inventa fpecimina dantur adhuc follilia calcaria ex lapicidina petrefactorum memorabilium plenifima montis S. Petri prope Trajectum ad Mofam, in matrice calcaria parum compacta, fæpe fatis friabili, linc inde ferruginea, ideoque mox alba, mox lutefcente, ex qua facili negotio erui pollunt, variæ magnitudinis, uti nobis conftat, a $\frac{1}{2}$ lin. usque $2 \frac{x}{2}$ lin, in diametro cum aculeis, $\&$ horum numero a $3-9$, corpore valde globofo, granulis infignioribus, quam in naturalibns, partim etiam minutis. Singulari horum teftaceorum folfilium, nunc valde rarefcentiun fructura, fpeciem afteriæ extrinfecus quam maxime mentiente, qua etiam nobis olim, uti ingenue fatemur, impoluit, celeberrimi lithologi \& Conchyliologi commoti, ifta corpuscula pro Afteriis $f$. Rellis marinis agnoverunt \& venditarunt, (vid. Walch. Gefch, d. Verft. Th. 2. Abfchn. 2. p. 295. No. S. ad Knorr. Tabb. Supplem, tab. VI. * fig. 8-17. Sclvöter Einleit, in d. Kenntn. d, Steine u. Verftein. Th, 3. p. 374. N. 12. tab, 5. fig. S. 9. qui ea ad Afteriam aculeatam Limu, retulit), fed maxima horum foffilium cum naturalibus Spenglerianis conformitate, præfertim interna fructura arte diffectorum infpectione folicita plene convicti fumus, illa non ex afteriarum genere, fed veras teftas Spirales involutas polythalamias, ergo Nautilos, \& quidemSpenglerianos effe.
2. Aumerk. Nebft diefen in ihrent natiirlithen Zuftande gefundenen Beyspielen giebt es auch noch kalhfleinartige folfile aus dem on merhüurdigen Verfeinerungen ïberaus reichen Steinbruche im $p_{e}$. tersberg bey Maftricht, die in einem etwas lockern, oft siemlich serreiblichen, hin und wieder eifenfshịfigen, daher bald weifsen, bald gelblichen Kalkfteine liegen, aus welchem fie mit leichter Miihe herausgenommen werden lönnen, vonverSohiedener Gröfse, foviel wir hennen, von $\frac{1}{2}$ bis $\approx u 2 \stackrel{2}{2}$ Linien ind Durchmeffer fonmt den Statheln, und diefe zon 3 bis 9 an der Ansahl, vou ftark hugeligen Leibe, uit anfehnlichern Kumnern; als an den natürlichen, wun Theil auth mit gans kleinen befetat. Duroh den gans lefonders Buu divfer foffilen jetst inumer feltner werdenden Sclineckshen, deffen Äuferliches nit ciner gewifen Ant, Seeflerne viberans viel Ahulichieit, und aush ehomals uns, wie wir offenheraig bejernen, hiniergaigen hat, find fehr beruhnte Verfteinerungs- unid Conshylienhemer veranlafit worden, jene Kürper-hen fïr Seeftcrne anzufehen und auswugeben. ( $S$. Walch Gefsinchte d. Vergein. Th. 2 Abfhir. 2. S. 29j. N. 8. au Knorrs PetrefastenWerk, Supplenent Taf. VI. * fig. S. 17. Schröter Einleit. in die Kenntn. der Steine und Verflein. Thl. 3. S. 374. No. 12. Taf. 5. fig. 8. 9. wo fie zur linneiSchen Aflevias aculeata gerechnet find). Allein durch die ïberaus grofse Ulereir- 
finnunig diefer fofflen Rürper mit den natiirlitherz Jpenglerijchen, und vorsiglich durch forgfältige Einficht des innern Baues kiinflich geöffneter find wir wollkommen iiberseugt worden, daß fie heine Secferne, fondern wahre fpiral eingerollte vielkrmmerige Schnecken, folglich Schiffer, und awor spenglerifche, find. .

\section{NAUTILUS PLANATUS.}

\section{DER FLACHF SCHIFFER.}

T A B. 16. Fig. $a-i$.

VARIETAS ERSTE ABÄNDERUNG.

T A B. 16, Fig, a. b.c.

Schröter Neue Litterat. d. Naturgefcb. I. Bd. p. 3I4, tab. I. fig. 7?

$T_{\text {ESTA fpiralis involuta, fubovalis, }} D_{\text {ie }}$ SCHALE iff fchnirkelformig einutrinque planata, unbilico parvo, pa- gerollt, beyzahe oral, auf beyden Seiten rum profundo; dorfo licet tenui, tamen flach, hat einen kleinen etwas vertieften obtufato, anfractibus repente crefcenti- Nabcl; der Rücken ift swar diinn, aber bus duobus, cxtimo folum confpicuo; doch nicht fchneidend, Sondernetwas funnpf; 
articulis, (fub quibus thalami) levifime trausverfim ftriatis, circa viginti, ftriis oculo armato vix vifibilibus; ifthmis antrorfum convexis, ad partem teftæ interiorem magis arcuatis; verfus dorfum f. marginem exteriorem rectiusculis, (vid. fig. i. hujus tabula); plano orali lineari, totum latus anterius ultimi articuli conftituente, utrinque leviter marginato; orificio longitudinaliter per medium plani oralis totum protenfo anguftifimo lineari, loco aperturæ fimplicis, ex foraminulis minutis interruptis compofito.

COLOR albus.

PATRIA litus ad Libumum portum in Hetruria.

MENSURA $\frac{2}{3}$ lin, long. \& $\&$ lin, lat. die Windungen, deren wur swey worhanden find, aber nur die äufsere fichtliar ift, nehmen fchnell an Grö/se au; die Glieder (unter welchen die Kanmesn liegens) ungefuilr arvansig, find fehr feitht erhaben und in die Quere Jehr fein geftreift (fo days die Streifeni kaum von einem bewaffneten Auge bemerkt werden kömnen); die Scheidewände find vorwärts gewölbt, an dem innern Theil der Schale ftärker gehriimmt, gegen den Rïchen oder den äufsern Rand zu etwas gerader (S. Fig. i. diefer Tab.); die Mündungsfläche ift frichformig, nimmt die ganze vordere Seite des letzten Gliedes ein, und hat cuf lieyden Seiten einen fohwachen Rand; die Mündung if nach der Länge mitten durch die ganze Minndungsfliche ansgeAreckt, Jehr Jchmal, ftrichformig, und ftat einer einfaihen Öfnung beftehet fie aus vielen durch gleichweite Zivifhenwände algefouderten kleinen Löchern.

$F A R E E$ weiss.

$V A T E R L A N D$ die Kiifle bey Livorno in Toscana.

GRC̈SSE $\frac{2}{s}$ einer Lin. long, und $\frac{1}{2} \mathrm{Li}$. nie breit.

Nota. Dni Schröteri exemplar huc pertinere videtur, licet tres vel quatuor anfractus ei attribuerit, (citata icon 7. tamen folummodo duos \& dimidiun monfirat) noftrum vero exemplar non nifi duos habeat, uti ex icone fpeciminis hujus va.
Anmerk. Herrn Schröters Exemplat. fcheint hierher an gehören, ungeashtet or in feiner Befarreibung der Schale drey bis vier. Windungen beygelegt (doch find in der angefiihrten Figur blofs swey und et.

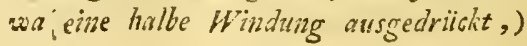


riclatis in nofira tab. fig. i. teftam per miner St iick aber nuv zuvey hat, wie aus axin majorem (vulg. horizonta'iter) dimi- der auf unferer Tafel fig. i. gelieferten diatam reprefentante, fimulque cam to. Albildung cines Beypiels dicfer Vurietam in viginti thalamos divifam elle, tät, das die Schale durch die grö/sere evidenter apparet. In ceteris omnibus Axe (insgennein horizontal) halb angeambo exemplaria inter fe conveniunt. fohliffen vorflellet, und sugleich auch das deutlich erhellet, days die gaize Sihale in zwansig Kammern getheilet fey. In aller iibrigen Stïmen hingegen komnen die beyden Beyspiele mit einnander überein.

VARIETAS

ZWEPTE ABÄNDERUNG.

T A B. 16. Fig. d. e. f.

Differentia inter hanc \& præcecientem

Dor Unterfchied wwifhen diefer und in eo confiftit, quod tefta hujus late ex- der vorhergehenden beruhet duranf, duß panfa antrorfumque valde convexa, at- diefer ihre Sshale fark ansgebreitct und tamen angulo exteriore feu dorf parum vorwiorts fark gewülbt, doch am äufern productiore \& levifime recurvato lateribusque fubflexuofa fit, fed utrinque umbilicum concarum cum ifta communem habet.

COLOR \& PATRIA ut fupra. MENSURA $\frac{x}{3}$ lin. long, \& lat. oder Rüclenvinkel ein wenig hinausftehend, und dabey fehr feicht ausgebogcin, auch an don Seiten etweas hin wald her gebogen ift; hingegen einen revtieften $N_{a}$ bel auf jeder Seite mit jener gemein hat. FARBE u.VATERLAND wie oben. GRÖSSE : Lin. lang und breit. 


\section{T A B, 16. Fig. g. h.}

Tefia quidem cum prima varietate in

Der Bau der Schale lonmt awar mit loc convenit, quod longiuscula \& rec- der erfen Varietät darin iiberein, daj to-plana (non flexuofa ut var. B.) fit \& fie etroas länglich zund gerade geebnet regulariter fpiralis usque ad medium ipfi- (nicht hinz und her gelogen, wie die ate z1s, fed a duobus pracedentibus fe di- Var.) und bis zur Mitte der Lïnge refinguit in eo, quod ab ifto medio quafi gelmäfsig Jpiral gevonnden ift; hingegen abrupta \& coarctata de novo velut inci- unterfsheidet fie fich von beyden workerpiat \& pedetentim iterum regulariter gehenden dadurich, dafs fie in gedashtcr antrorfum convexe fefe late expandat, Mitte wie abgebrochen und wiel folmailer exceptis articulis f. thalanis duobus ul- oder vercugert and gleichfan neu anfontimis, qui in medio latitudinis aliquan. gend erfsheinet, und fich wiederum nach tum retracti \& fubflexuofi evadunt.

COLOR \& PATRIA ut antea. und uach regclmaifsig vorwairts gervjlbt weit auslveitct, die leyden let:ten Glicder oder Kanmer'n ausgenommen, die in der Mitte der Breite ein wenig rickwiirts - gedriicht, oder verf:hmillert und faft geradlinig fich aeigetr.

FARBE und VATERLAND wie vorige.

MENSURA $\stackrel{x}{s}$ lin, long.

GRÖSSE $\frac{1}{3}$ Linie lang. 


\title{
NAUTILUS CASSIS.
}

\section{DER HELMFORMIGE SCHIFFER.}

T A B. 17. Fig. $a-$ l. \& T A B. 18. Fig. a. b. c.

Soldani Teftaceogr. T. I. P. I. p. 63. tab. 55. fig. A-G. E tab. 56. fig. $H=N$.? Nautilus lituitazus, apud Modeer orthocera crifpata \& Orbiculus.

VARIETAS $\propto$. ERSTE ABÄNZIERUNG.

\author{
T A B. 17. Fig. a.b.c.d.
}

Jan. Planc. Conci. p. 120, $2 a b$. I. fig. II. Q. R.

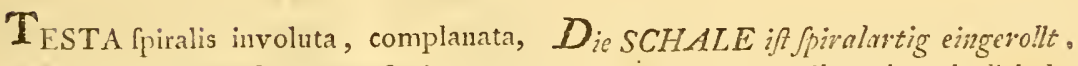
femiovalis, parte fcilicet pofteriore ro- beyderfeits fach, halb oval, nehmlich dev tundata, anteriore fubllexuofe truncata; hintere Theil sugerundet, der vordere dorfo acute carinato, margine lato mem- etwas logig abgefutat ; der Rücken branacco, fubrepando; anfractibus la- foharf gekielt, mit einen hantformig dïn= vibus; articulis levifime clevatis; ifth- nen, aber lreiten, etvas ansgefinweiften mis flexuofe antrorfum convexis, extus Rande; die Windungen find glatt; die prominulis, loco umbilici granulis pro- Glieder fehr Sthwach erhöhet, die Scheimiscue coacervatis, rotundis \& oblon- dewände hin und her gebogen, voruarts gis, inæqualis magnitudinis, medio gran- convex, aufsen feicht hervorragend; an diore; plano orali lineari, longitudinis der Stelle, wo fich fonft der Nabel befinaqualis latitudini totius tefix levifime det, fitsen "etliche ruude und lifigliche ohSubcarinato, utrinque parum marginato; ne Ordnung sufanmen gehüufte Körner orificio lineari f. rimula margine conve. verfuchiedener Grö/se, seozoin das antitel. 
xo ovali crellato parvo prominente in $f e^{\circ} d \alpha s$ gröfste iff; die Mündungsfläche, plani oralis angulo externo, a quo ori- deren Lïnge der ganzen Schalenlreite ficio longitudinaliter excurrit rimula dicti gleich komnt, ift frichformig, fehr fach orificii, longitudine fenfim dilatata, ob- gehivlt, beyderfeits mit einem kleinen tufe claufa (vid. fig. c., cujus pars ma- Rande eingefafst; in derfelben änfsern gis acuta fig. d.) in angulo oppofito au- Winkel befindet fich die frichförmige tem lamina five dente compreflo obtufo. Mündung fellft, oder cine Kleine Spalte, In angulo extcrno quinque fexve articu. mit einer ovalen gevölbten und gekerb. lorum anteriorum obfervantur aliquot ten Einfoffing, fie ift klein und hervorcrenulæ obfoletæ quafi veftigia vel reli- ragend, won welcher Mündung nach der quiæ orificiorum junioris tefiæ fenfm ar. Lainge eine kleine Spalte auslänft, die ticulis graudefcentis. So lang als die Mündung if , fich allmählig erueitert und fich fumpf endiget ( $S$. fig. c., ein noch mehr aergröfserter Theil fig. d.), inn entgegen gefetsten Winkel aber feht ein Plattchein, oder ein $\approx u-$ fanmen gedrïinter ftumpfer Zahn. An finf bis fechs wordern Gliedern leobuchtet man in äufsern Winhel einige verlochene Kerlen als gleichfan spuren oder Ulerbleilel von Mindungen der jünger Schale, die nach und nuch durch Anjetzung mehrever Glieder gröfser angewachjen ift.

COLOR albo-flavefcens.

PATRIA Coroncina prope Sellam Hetrurix.

MENSURA ${ }_{3} \frac{5}{3}$ lin. long. \& lat.

$F A R B E$ weifsgelblich.

$V A T E R L A N D$ Coroncina bey Siena in Tosina.

GRÖSSE $1 \frac{2}{3}$ Linie lang und breit.

Nota. Hanc fpeciem quiclem Janus Plancus fupra cit. loco nomifr varietatem Nautili Calcaris elle fibi perfuafit; fed quicunque tefulas noftras attento infpecturus animo difierentiam fpecifican

Anmerk. Diefe Art hat wuar Fanus Plansus an olen angefiihten Orte fïr - cine blofse Atrinderung 'vom Spornf-hiffer angefohen; allein wer nur unfere Schneckchen anfmerhfou betrachten wird, der 
inter hanc aliasque fpecies negare non wird ats denffeciffchen Unterfichied swipoterit, licet confitendum, eam ad Nall- Schon diefer und andern Arten nicht in tilum Calcarmultum appropinquare. Hæe Abrede ftellen lïnnen, ob nian greich be. ıefulæe a latere adfpectæ, magnitudinem lennen mußs, daßs diefe Art fich dem Sporn. fi demas, aliqualem fimilitudinem habent fehiffer siemlich nuhere. Wenn nan diefe cum caflidis vel galeæ quadam fpecie, kleinen Schalent zon der Seite anfiehet und id guod interim hujus fpeciei fupra ciic- fich die Grüßse weg denht, fo fundet masz to modo denominande nobis anfam de- einige Ähnli-hheit awifchen ihnen und eidit; articuli vero velut lamina cataphra- ner Art He'm oder Sturmhaube, welches ctæ fibi invicem fubimbricatæ f. fubimpo- uns anch inzwifshen Anlafs gegeben hat, fitæ videntur.

diefer. Art den obigen Nahuren au geben; die Glieder felbft aber Solreinen wie Schienen eines Harnif:hes etwas iiber einander liegend oder in einander gefshoben an seyn.

VARIETAS $\beta$.

ZWEYTE ABÄNDERUNG.

\section{T A B. 17. Fig. e. f. g.}

Soldani Saggio orittogr. pag. 97. tab. 1. fg. 1. A. B. C. (affinil.)

Differt a pracedente in eo, quod fatis oblonga \& diffepimenta in tefiz parte dimidia pofteriori granulis obfita, in dimidia autem parte anteriore lavia fint, iftaque tefiæ pars ifilhmis granulatis prædita fatis ventricofa $f$. utrinque convexa $\&$ orificium non in rimulam productum fit, fed per fuum medium longitudinale apertura fubovata elongata gaudeat.
Unter.sheidet fich von roriger dadurch, daf: fie siemils verlangert, und die Sohieidewcinde anf der lrintern Hailfte der Sihale wit Künnern befetat, auf der vordern Hilfte aler glatt find, auch jener nit gekürnten Scheidewcinden vergehene Theil der S:hale zientich bauthig oder auf beyden Seiten gewillbt, und die Miindung in leine Nelenspalte verlaingert ift, fondern nach der Länge mittendurch etwas eyfor. mig veriangert offen fichet. 
COLOR proprie albefcens, ab iuclufa terra ferruginea fufcefcens.

PATRIA Coroncina.

MENSURA 2 lin. long. $1^{\frac{\pi}{2}}$ lin, lat.
FARBE eigentich weifslich, won der eingefchloffenen eiferafchiiffigen Erde aber bräunlich.

$V A T E R L A N D$ Coroncina.

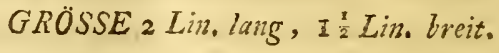

VARIETAS $\gamma$.

DRITTE ABÄNDERUNG.

T A B. 17. Fig. h. i.

Hæe varietas difcedit a prioribus hoc modo: præter marginem fatis latum fubdentatum omnes ifthmi crafliores valde eminent \& granulis oblongis fibi invicem oblique contiguis obtecti, funiculi inftar quafi contorti, apparent; tefia etiam craflior eft, quam in reliquis.

COLOR teftæ albo-flavefcens, granulorum cærulefcens.

PATRIA Coroncina in Hetruria.

MENSURA I lin, long.
Diefe Abänderung weicht zon vorigex folgender Weife ab: aufser dem, dafs der Rand siemlich brcit und einiger Maysen geaïhnelt ift, find alle Zwifchenwände aufsen ziemlich dich nnd fark hervorragend und mit langlichen fchief an einander liegenden Könern dergeftalt befetst, dafs es das Anfehen hat, als wären fie nit gewnndenen Strisker: belegt; die Schale ift auch dicher, als an den andern. FARBE der Schale weifsgellich, der Kömer blaulich.

VATERLAND Coroncina in Tos. catla.

GRÖSSE 1 Linie, lang. 
VARIETAS $\delta$.

VIERTE ABÄNDERUNG.

\section{T A B. 17. Fig. k. $\%$}

Soldani Tefaceogr. T. I, P. I. p. 63. tab. 55. fig. A. ? (afjimil.).

Differt a ceteris in eo, quod tefia fit lævis, nullis granulis prædita nulloque umbilico, fed hujus loco folummodo levifima imprefio. Ceterum, ut prima \& recunda, margine lato fubrepando gaudet.

COLOR \& PATRIA ut fupra.

MENSURA I $\stackrel{\perp}{4}$. diam.
Diefe Varietät geht von den iibrigen darin ab, daß die Schale ganz glatt, ohne einige Korner oder andere Erhöhnugen ift, auch keinen weder erhöheten noch vertieften Nabel hat, fondern bloß anf der Stelle, wo fich diefer jonft befindet, eine felr feichte Vertiefung $\approx$ ub berken ift. Ubrigens hat fie, wie die erfte and srveyte, einen breiten etwas ansgefchrveiften Rand.

FARBE und VATERLAND wie oben.

GRÖSSE 1 \& Linie in Durohme Jjer.

VARIETAS $\varepsilon$.

FÜNFTE ABÄNDERUNG.

T A B. 19. Fig. a. b. . .

Differentia in hoc confifit, quod tefa hac in loco umbilici aliquot granulis prædita, præfertim vero planum orale longitudinaliter valde coarctatum five breve, $\&$ in angulo interiore, feu qui orificio crenato oppofitus cft, dens fubtriaugularis compreffus adfit. Et hic ori-
Der Unterfchied liegt darin, daßs bey diefer S:hale on Platze des Nabels nur etlishe Kümchen arorharden find, befonders aler die Mündungsfliche nach der Liunge genonmen fark verengert, oder kurz, und in den innern oder den der gererbten Mindung entgegen gefetaton $\mathrm{N} 2$ 
ficium non nifi incifura longitudinalis in. Wintel ein aufanmen gedrückter. faft ter crenas elt.

COLOR albefcens.

PATRIA ut fupra.

MENSURA $2 \frac{3}{4}$ lin. long. \& $1 \frac{\pi}{3}$ lat. dreyeckiger Zohn befindlich ift. Auch hier beftehet die Mündung nur aus einem zwiJchen feinen Kerben nach der Länge laus fenden Einflinitt.

FARBE weifslich.

$Y A T E R L A N D$ wie oben.

GRÖSSE $2{ }_{4}^{x}$ Linie lang und $1 \frac{\mathrm{T}}{2}$ breit.

\title{
NAUTILUS GALEA.
}

\section{DER STURMHAUBENFÖRMIGE SCHIFFER,}

\author{
T A B. 18. Fig. d. e. f.
}

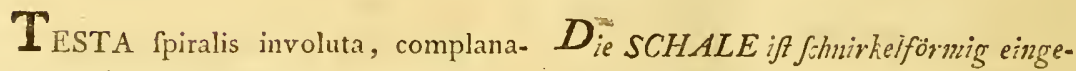
ta, lavis, exumbilicata, fubfemiovalis, rollt, völlig flach, glatt, olme Nabel, nempe parte pofteriore rotundata, ante- faft halboval, nehunlich an hintern Theile riore fubflexuofe truncata; dor.jo acute zugerundet, vorn etwasbogig algeftutat; carinato, margine lato membranaceo der Rücken ift fcharf gekielt, mit einem repando; mefractibnis levibus ( $\mathrm{I}-2$ ), ex breiten hautformig diinnen ausgefchweifquorum articulis leviter elevatis extus ten Rande; die Windungen find glatt tredecim confpicuis dimidia pars five fex $(1-2)$, aus ihrenfchwach erhabenend dreyulteriores a reliquis parum disjuncti funt; zelnn von aufen fichtbaren Gliedern find iffhnis antrorfum convexis, mediocriter die fechs letatern als die Hailfte der iibricurvatis; plano orali lineari levillime ca- gen ein wenig abfehend; die Scheidewänrinato, longitudine æquali latitudini to- de haben ihre Wölbung vorwärts, fird 
tius teftr, in cujus angulo externo orif- mittelmàßig gelogen; die Mündungsfäcium tefta \& fimul fiphonis lineare an- che ift frichformig, fehr wenig gekielt, guftifimum f, rimula longitudinalis, mar- ihre Linge gleich der Breite der ganzen gine crenato ovali.

COLOR albefcens.

PATRIA Coroncina- in Hetruria.

Schale; in ihrem iulßern IVinhel ift die Mündung der Schale und sugleich auch der Nervenröhre frichförmig fehr fuhnal oder eigentlich eine Spalte nuch der Länge nit einer ovalen geierbten EinfafJung.

F-ARBE weifslich.

VATERLAND Coroncina in Tos. cana.

MENSURA $1 \div$ lin. long. \& 1 lin. lat.

GRÖSSE ${ }_{\frac{1}{4}}^{2}$ Lin. lang $u$. I Lin. Ureit.

Nota. Defcriptionem plani oralis $\mathcal{S}$ Anmerk. Die Befithreibung der Miin. orificii folummodo dubie ex ftructura penultimi articuli, ultimo partim diffracto, defumptimus, quia hæc ftructura in omnibus articulis feu thalamis eadem eft, uti non folum ex fractura ultimi thalami, fed etiam ex difepimentis reliquorum thalamorum, quoniam tefta pellucida eft, apparet.

Hæc fpecies parum differt a Nautilo Caffide, \& non nifi thalamis disjunctis; quapropter etiam ob maguam fimilitudinem Caffidis cum Galea ultimum nomen ei attribuimus. dungsfache und Mindung haben wir blos aweifellhaft vom Bau des vorletwen Gliedes, da das letate anm Theil weggebrochen if, hergenommen, weil diefer Bau an allen Gliedern oder Kanmern einerley ift, wie folches nicht nur aus dem Bruch der letaten Kammer, fondern auch aus den Scheidewünden der iibrigen Kammern, indem die Sihale durch/ichtig ift, deutlioh erhellet.

Diefe Art weicht fehr wenig and blo/s durch die abfehonden Kamunern vom helmförmigen Schiffer ab; daher wir anch, wegen der ftarken Ähnlichreit sines $\mathrm{Hel}$ mes mit einer Sturmhaube, ihr den letzterw Nahmen beygelegt haben. 


\section{NAUTILUS AC'UTAURICULARIS.}

\section{DER SPITZOHR - SCHIFFER. \\ T A B. 18. Fig. g. h. i.}

Soldani Teftaceogr. T. I. P. I. p. 6r. tab. 49. fg. X.? (ridditcr affinil.).

\section{TESTA fpiralis involuta, fubovalis,} lævis, ventricofa, exumbilicata; dorfo acute carinato; anfractus exterioris articulis decem confpicuis fubelevatis; diffepimentis mediocriter antrorfum convexis; plano orali ovato convexo, marginato, angulo exteriore coarctato prominente obtufo fubincurvato; orificio in dicti anguli extremitate rotundo minuto (lit, o.).

Propter magnam affinitatem cum $N$ ruttilo Auricula (tab. 20. fig. $a-f$.) a quo nonnifi plano orali \& angulo prominente coarctato, quibus duobus criteriis dictus Nautilus caret, \& tandem thalamis pluribus differt, buic fpeciei altefatum nomen dedimus.
Die SCHALE ift spiralförmig eingerollt, siemlich oval, glatt und bauchig; der Rïcken fchurf gehielt; von der ainfsern Windung find zehiz Glieder fichtbar und ein wenig erhabeu; die Sclieidewände vorwairts mittelmaifsig gewülbt, die Mündungstläche ift eyformig erhaben gewöllt, mit einem kleinen Rande eingefafst, ihr ciufserer Winhel verengert, hervorragend, abgeftumpft und etwas eingenriimmet; die Mündung befindet fich an Ende gedachten Winhlels, iff rund und klein (lit. o.).

Wegen der grofen Vervondt fhaft mit dem ohrförmigen Schiffer ( $t a b$. 20. fig. a-f.) von welchem gegennuirtige Art blofs durch die Miundungsfiche und durch den hervorragenden verengerten Winhel, welche beyde Kennseichin gedachtem Schiffer fehlen, und endlith duroh die mehrern Kamunern fich unterfiheidit, 
Cum Nantilo Crepidula (tab. 19, fig. g. haben wir derfelben oben erwihnten Nohh. i.) quoque magnam habet fimilitudi- men gegeber.

nen, fed difcedit ab eo præcipue ftruc- Mit den Pantoffel-Schiffer (tab. 19. tura multo breviori, at ventricofiori, fig. $g . h . i$. ) hat diefer Schiffer gleichfalls carina acuta, paucioribusque atque ele- viel Ähnlicheit; er unterf.hcidet fich aber vatioribus thalamis. vou ihm haufteichlich durch feincn viel küraern, dagegen bauchigern Bau, durch den fohafen Kiel, und durch wenigere, aber erhabnere Kammern.

COLOR albefcens. FARBE weifslich.

PATRIA concretiones Zoophyticæ maris mediterranei.

MENSURA $\frac{x}{3}$ lin. long. plusquam ${ }_{4}^{x}$ lat. \& $\frac{3}{6}$ crafl.

VATERLAND Zoophyten - Concretionen des mittellandisinen Meres.

GRÖSSE $\frac{x}{3}$ Liñ.lang, mehrals $\frac{x}{4}$ brcit, und? dick.

\title{
NAUTILUS FABA。
}

\section{DER BOHNENFÖRMIGE SCHIFFER.}

\author{
T A B. 19. Fig. a. b. ..
}

Jan. Planc. Couch, p. 13. sab. I. fig. 4. L. Mi? (minas accurate).

Ledermüller Microfcop. tah. 8. fig. d.? (iremi).

Martini Conch. Cab. P. I. tab. 19. fig. 170? (itemb, ex Ledcrm.).

Soldani Saggio oristogr. p. 101. tab. 2. fig. 18. (foljil. ifthmis autrorf, coulcav.).

Testa fpiralis involuta, fubovalis, utrinque fubcomprefla $\mathrm{f}$, mediocriter convexa, exumbilicata, medio folummodo

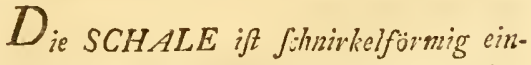
gerollt, wientich oval, auf beyden Seiten etwas zufammen gedrickt, oder mit- 
leviter impreflo; dorfo pofice acute carinato, antice magis obtufo; articulis tredecim confpicuis levibus planatis, ita tut fitus iffhmorum extus non aliter difcerni polfit, ac colore obfcuriori lateribus eorum refpondente eosque antrorfum nediocriter convexos effe indicante; plano orali fubcordato convexo, in cujus latere interiore orificium fublineare para. bolice arcuatum, marginatum, interruptum $\int$. ex foraminulis circiter octo uniferiatis compofitum.

COLOR albefcens, vel flavefcens, etiam cinerafcens, præfertim folfilium.

PATŔlA naturalium præcipue arena conchyliofera littoris ariminenfis aliæque regiones maris adriatici $\&$ mediterranei; foriles D. Abbas Soldani invenit in terris Seneufibus, Volaterranis, in loco dicto Poggio di S. Anfano prope Caftrum S. Quirici, \&c.

MENSURA, ut nobis confiat, $\frac{3}{6}-\frac{1}{3} \mathrm{lin}$. Exilitas ejusmodi cochlearum ex eo colligi facile potefi, quod fecundum Dni. Soldani allertum 500 foliles vix pondus grani triticei adæquent. telmaifsig gewölbt, ungenalelt, blofs ins Mittelpunct gans feicht eingedriisht; der Rücken if hinten finarf gehieit, vorn mehr fumpf; die dreyzehn fichtlaren Glieder find glatt und fach, fo dafs man die Stellung der Scheidewäncle von ath Sien nicht anders unterfsheiden hann, als durch eine dunhlere Farle, die ihren Kan. ten entfricht und anseigt, dafs fie vorwärts mittelmajsig eihalen find. Die Mündungsfäche ift einiger $M a / s e n$ herzförnig und gewölbt, an deren innerer Seite seigt fich die beynahe frrichfurmige Mündung, die parabolif:h gehrümmt, nit einer Einfaffung verfehen und unterlrochen, oder aus nugefahr acht in einer Reihe liegenden hleinen Lö:hern aufammen gefetat ift.

$F A R B E$ weifslich, oder gelblich, ansh graulich, befonders an den folfilen.

$V A T E R L A N D$ der natürlichen vorsiiglich der Mufshelfand ann Strande bey Rimini, und andere Gegenden des adriatifihon und mittellaindifochen Meeres; foffil hat fie Hr. Abt Soldani gefunden bey Siena, Volterra, Poggio di San Anfano unrucit des Schlofjes San Quirico Eve.

GRÖSSE, fo viel ielannt, :- $\frac{1}{3}$ einer Linie. Die Kleinheit diejer Schneckchen laifst fich finon daraus leicht alnehmen, dafs, nach Hrn. Soldani Vorge. ben, 500 von den folfilen naum die Silhuere eines Weizgnhorns letragen. 
Nota. Hæ teftula, quibus ab earum forma aliquo modo fabæ fimili, hujusmodi nomen tribui poteft, ad Nautilum Pompilizm \& Pompilioiden multum ac. cedunt, fed fitu thalamorum ifthmorumque antrorfum convexo (qui procul dubio ex errore fculptoris in Soldani icone ratione inverfa fcil. antrorfum concavi repræfentantur) formaque magis comprefla $\&$ dorfo acute carinato potifimum $a b$ iis difcedunt, quorum prior ifthmis antrorfum concavis gaudet, ambo vero forma valde ventricofa dorfoque rotundato præditi funt.
Anmerk. Diefe S:hnechchen, denen, wegen ihrer einer Bohne einiger Mafsen nahe hommenden Geftalt, dergleichen Nahme beygelegt werden kann, nähern fich Jehr dem dickfchaligen und dem kleinen dicken Schiffer; allein durch die vorwärts gewölbte Stellung der Kanmern und Scheidewände (die vermuthlich aus Verfehen des Kupferftechers in der Soldanifchen Abbildung ungelehrt, d. $i_{0}$ vorwarts hohl vorgeffellt find) wic auch durch die mehr zufammengedriitite Ge. falt und den l:harf gekielten Riiivien, weichen fie hauptfichlich won jenen ab, won welchen erfterer vorwairts hohle Scheidewcinde, beycle aber eine fark baushige Geftalt und einen runden Riionen haben.

\title{
NAUTILUS SCAPHA.
}

\section{DER KAHNSCHIFFER,}

\author{
T A B. 19. Fig. d. e. $f$.
}

IESTA f́piralis involuta fubovalis utrinque umbilico impreflo parvo, lateribus rollt, faft oval, hat beyderfeits einen mediocriter convexis (minus quam in Weinen eingedrïohten Nabel und nittel- 
præcedente Naut. Faba, fed magis quam in fequente Naut. Crepidula); dorfo obtufo; articulis duodecim confpicuis, fubelevatis, levibus; difepimentis antrorfum mediocriter convexis, non omnibus ex centro radiantibus, fed tribus ultimis parum extravagantibus; planto orali oblonge-fubcordato convexo; orificio (in fpecimine obvio unico partim detrito) prafumtive, uti in aliis fimilibus, lincari parabolice arcuato.

COLOR albus.

PATRIA mare adriaticum.

MENSURA \$ lin. diam. maj. máfsig gewjibte Seiten (weniger als bey vorgehenden Bohnenflhiffer, ftürker aber als beym folgenden Pantoffels:hiffer); der Rücken ift fumpf; die zwjif fichtbaren Glieder find etwas eriobliet and glatt; die Scheidewände vorwärts mittelunäfsig gewölbt, lanufen nicht alle frrahleanceife aus dem Mittelpunste, fondern die drey letsten Sohweifen ein wenig daron ans; die Mündungsfläche ift lainglich aientich hersfornsig und convex; die Mündung (die am gegenuärtigen einzigen Exemplar sum Theil verdorben ift) mag vermuthlich, wie bey andern von ähnlicher Bauart, frrichformig und parabolijch gebogen Seyn.

$F A R B E$ veifs.

$V A T E R L A N D$ das adriatifohe Mfeer. GRÖSSE $\frac{x}{3}$ Linie im längern Durchmeßer. 


\title{
NAUTILUS CREPIDULA.
}

\section{DER PANTOFFEL ÄHNLICHE SCHIFFER.}

\author{
T A B. 19. Fig. g. h. i.
}

Soldani Tefaceogr. T. I, P. I. pag. 64. ta'. 58. fig. bb.? Nautilus Litnitatus.

$T$

TESTA fpiralis fubiuroluta, elongata, leviter curvata, lavis, pellucida, compreffa f. lateribus fubelevatis (adhuc compreflior, quam in præcedente Naut. Scapha); dorfo obtufo; articulis 12-13 confpicuis, planatis, excepto ultimo elevatiore; ifthmis leviter antrorfun convexis e communi centro radiantibus, duobus tribusve ultimis vix extravagantibus; flano orali lanceolato convexo; orificio minuto rotundo leviter crenato in externo ejusdem angulo.

COLOR albidus f. margaritaceus.

PATRIA litus liburni portus in Hetruria.

MENSURA $\frac{s}{3}$ lin. long.
$D_{\text {ie }}$ SCHALE if fihnirhelformig sum Theile eingerollt, in die Länge geftresht, ein wenig gebogen, glatt, durchichtig, zufanmengedriicht, oder mit wenig erhoheten Seiten (viel mehr sufammen gedriicht, als beym vorhergehenden KahnSchiffer); der Rücken ift ftimtf; die 12 - 13 fichtbaven Glieder find flach, ausgenommen das letate, das fich etwas crhelt; die Scheidewände find rorwärts feisht gewölbt und lanfen frrahlig aus dent gemeinfamen Mittelpunet, nur zwey bis drey der ietsten ausgenomuen, die kaum snev lich ausfchreeifen; die Mündungsflïche if lansettformig und gervälbt; in ihrem äufiern Winkel liegt die Mündung, die hein, rund und fein gekerlt ift.

FARBE wejilich cder ferlforl. $V A T E R L A N D$ die Kiifte bey Lizor$n 0$ in Toscana.

GRÖSSE $\frac{2}{3}$ Linie lang.

$\mathrm{O}_{2}$ 
Nota. Hac fpecies, Nautilos nofiros (Nautilos fpirales rotundatos Limn.) connectit cum Lituis \& Orthoceratibus (Nan.tilis elongatis Linn.).
Anmerk. Diefe Art macht cinen Ubergang von unfern Schiffern (Jpiralformig rund gewundenen Nautilis des Linné) zu des Krummftäben und Geradfäben (gerad gefrelten Nautilis des Linué).

\title{
NAUTILUS AURIC'ULA.
}

\section{DER OHRFÖRMIGE SCHIFFER.}

\author{
T A B, 2O, Fig. $a-f$. \\ VARIETAS $\propto$. \\ ERSTE ABÄNDERUNG. \\ T A B. 20. Fig. a. b. c.
}

Soldani Teftaceogr. T. I. P. I. tab. 50. fig. $Y$ ?

TESTA fpiralis fubinvoluta, ovalis, $D_{i e} S C H A L E$ ift fpiralfürmig, meilævis, utrinque fatis convexa, exumbili- fens eingerollt, oval, glatt, beyderfeits cata; dorfo acute carinato; articulis fep- zienlich gewölbt, ohne Nabel; der Rïtem coufpicuis fubelevatis, antrorfum cken foharf gekielt; von den Gliedern leviter convexis, ex centro radiantibus, find fieben fichtbar, etwas erhaben, vorexceptis duobus ultimis extravagautibus wärts fehwach gewölbt, und laufen frahlf. extra centrum inititum capientitus, ul- weife ans dem Mittelpuncte, bis auf die timi angulo initiali $\{e u$ interno, apice swey letatcn, die ans fchweifen, das ift, 
fubobtufo, parte autem oppolita exter- die au/serhalb dem Mittelpunte ihren Anna valde lata \& rotundata; de difepi- fang nehmen, unter welchen beyden das mentis fimile huc quadrans valet. Pla. letzte oder vorderffe am innern oder. Anmum orale lic locum non habet, quia ca- fangswinkel eine etwas zugeftumpfte Spirina dorfi ad dictum anguli apicem usque tse hat, an der entgegen gefetaten Seite producitur. Nec orificiuni diftinguibile, aber fehr breit und angerundet ift; von an rinula parva linearis transverfalis per den Scheidewänden gilt das hierher geangulum externum ultimi maximi ar- hörige Ähnliche. Eine Mündungsfläche ticuli?

findet hier wicht Statt, wei! der gekielte Riicken bis an die erwähnte Winkelfpitse fich erftrechet. Auch die Miindung ift nicht au erkennen, fie fcheint eine frrichformige kleine quer iiber den äu/sern Theil oder abgerundeten Winkel des letaten gro/sen Gliedes laufende Spalte au Jeyn.

COLOR fufcefcens, a terra ferruginea, qua liæc tefiula farcta \& tincta eft.

PATRIA Coroncina in agro Senenfi Hetruriæ, folfilis.

MENSURA $\frac{2}{3}$ lin. long.

FARBE brüunlich von der eifen/chüffigen Erde, womit diefe kleine Schale angefiillt und gefärbt ift.

$V A T E R L A N D$ Coroncina in Sienifchen in Toscana, ift aifo folfil.

GRÖSSE ₹े Lin. lang.

Nota. Etiam hujusce fpeciei cum Nantilo Crepidula \& Naut, acut - auriculari magna eft fimilitudo; fed a priori diftinguitur præcipue fructura breviori, aft convexiori, porro carina acuta \& articulis paucioribus, fed elevatioribus. De differentia hujus a Naut. acutauriculari jam fupra (ad, tab. 18. fig. g.h. i.) locuti fumus.

Anmerk. Auch diefe Art hat nit den Pantoffel- und Spitzohr-Schiffer viele Ähnichkeit ; von erfteren aber unterfcheidet fie fich vorziiglich durch den kiirzern, aber gewölbtem Bau, fodann durch den Scharfen Kiel, und durch weniger, aber etwas erhabnere Glieder. Von ihren Un2. terfhied vom Spitwohrfchiffer haben wir bereits oben (bey der Tafel 18. Fig. g.h. i.) geredet. 


\section{T A B. 20, Fig. d. e. f.}

Hrec varietas a priori parum discedit \& nomnifi in eo, quod fit proportionate brevior five aliquantum latior illa (nam fpecimen, cujus icon, in tab =0. fig. $a$. b. c. exftat, inter inventa exemplaria latifimum eft; dantur autem plura zraciliora $\int$. minoris latitudinis). Porro differt eo, quod fit rentricofior, articuli panlo elevatiores \& difepimenta magis curvata, inque tribus quatuorve poftremis articulis oblique difpofita, nimirum in uno latere a fe invicem diftantiora, quam in altero (fig. c. lit. oo. pp.qq.rr.)

COLOR pellucide albicans.

PATRiA ex concretionibus Zooplyytorum maris mediteranei, adhuc in fiatu fuo naturali, non mutato, cum prior int forlilis.

MENSURA $\frac{\pi}{3}$ lin. long.
Diefe Varietät weisht :on der erffes wenig nud nur darin ab, dafs fie ver. hailtnifsmäfsig kïrzer, oder etwas brei. ter ift, als jene (denn das Stiich, das auf der Tafel 20.,fig. a. b. c. algebildet ift, gehört au den breiteften unter den gefundenen, da hingegen die Johmilern häufiger angetroffen werdenz). Ferner iff fie bauchiger, die Glieder fond ein wenig erhabener, auch die Schcidewände gelirimmer, und bey den drey bis vier letwten Gliedern laufen fie fihräge, indem fie nehmlich auf der einen. Seite weiter: von einander abfehen, als auf der andern (fig. c. lit. oo. pp. qq. rr.).

FARBE durchischtig weislich.

$V A T E R L A N D$ unter ZoophytenConcretionen des mittellandifonen Meeres gefunden; if noch in feinem natiurichen unveriünderten $Z$ uftunde, da hingegen die erftere Varietiat folnl ift. .

$G R O ̈ S S E \div$ Linie lang. 


\section{NAUTILUS TUBEROSUS.}

\section{DER KNOLLIGE SCHIFFER.}

T A B. 2O. Fig.g. h. ‥k.

TESTA irregulariter fpiralis involuta,

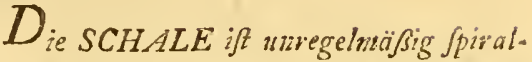
tuberofa, denfe punctata, punctis minti- förmig eingerollt, höckerig, nit fehr hleitiflimis excavatis, (non perviis, uti ex nen Puncten, dic hohl oder eingedriicht icone particulæ teftæ vitro valde auctæ (aber nicht durchgebrochen, wie die $A \dot{b}$ fig. $k$. patet) utrinque comprefa; dorfo bildung eines kleinen hierftark vergröfserobtufo; anfractu extimo confpicuo ex ten Theils der Schale fig. k. seigt,) find. aliquot tuberculis compofito, quare ejus articuli tredecim inæqualis magnitudinis \& forma funt, præfertim ultimus fatis magnus eft $\&$ ab altero latere valde prominens; diffepimentis antrorlum leviter convexis; plano ordi nullo; orifisio (lit. o.) lineari anguftiffimo fubtilifime marginato in margine partis interioris ultimi articuli, fecundum convexitatem dorfi articulorum minorum arcuato.

COLOR albidus.

PATRIA Coroncina in Hetruria.

MENSURA $x$ lin. long, \& lat. dicht befetst, nit aufannizen gepressten Seiten und fumpfen Rücken; die äuferfte fichtbare Windung beftchet aus etlichen Höckern, daher ihre dreyselnn Glieder von ungleicher Gröfse und Geftalt find, lefonders ift das letate sienlich grofs und auf der andern Seite fark hevorragend; die Scheidewände find vorwcirts fohwach gevö̈lbt; eine Mündungsfläche findet fich hier wicht; die Mündung (lit. o.) ift Arichformig, fehr enge, feln fein gefainnt, ant Rande des innern Theils des letzten Gliedes, nach der Wölbung des Rübleths der kieitiert: Glieder gelogen.

FARBE weifslich.

VATERLAND Coroncina in Tos. cana.

GRÖSSE L Linie lang und lreit. 


\title{
NAUTILUS ORBICULUS.
}

\section{DER SCHEIBENFÖRMIGE SCHIFFER,}

\author{
T A B. 21, Fig,a.b.c.d.
}

Testa fpiralis involuta orbicularis, fpiraliter firiata, friis multis parallelis a puncto eccentrico ad ultimi articuli fpatium finale approximato emanantibus, valde arcuatis, utrinque oblique fubcomprefla, difco eccentrico magis elevato; dorfo carinato in parte tenuiore, latiore vero lineari fubplanato in parte crafliore, ad finem (lit. o.) bifido, parte itraque arcuata, attenuata introrfum usque ad dictum punctum eccentricum prolongata; difepimentis antrorfum convexis; anfractuum duorum thalamis fubelevatis inultis (hic 28. interioris nimirum 18. \& exterioris 10. vid, icon, teftæ dimidiatæ fig. c.) angufits valde arcuatis, firiis externis refpondentibus, iterum per ifthmulos transverfos numerofos in loculos minutos fnbequilateros fubdivifis. Orificium in bac fpecie nondum detectum.
$D_{\text {ie }}$ SCHALE ift Spiralförmig, in fich selbft gewnnden, kveisrund, mit vielen parallelen ftark gebogenzen Streifen verfehen, die von einem ausserhalb des eigentlichen Mittelpunctes befudlichen, der EndAaishe des letwten Gliedes mehr gendiherten Puncte fpiralartig auslaufen: die beyden Seiten find fhief etwas aufanmen gedriicht, indem nehmlich die eccentrif:he innere Soheibe ftürker erhuben ift, als der iibrige Theil; der Rücken ift am diunnern Theil kielformig, an dichern Theil aber breiter, frichformig, beynahe flach, und wo er fish endiget (lit. o), theilet er fich in swey Theile und verlaingert fich mit diefen allmaihlig fich verdïnnernden gebogenen Theilen einwairts bis zum oben geuannten ecientrifchen Punst; die Scheidewände fand vorwairts convex; die Kammern der beyden Windungen find ein we. nig erhaben, hainfig, (hier 28, nehmlish an der innern Windung 18 , und an der äußeru 10; man fehe die Abbildung einer. 
sur Hälfte getheilten Schale fig. c.) enge undfark gebogen, mit den äufsern Strei. fen iibereinftimmend uzd abermahl durch zahlreiche kleine Querwände in kleine - zienlich gleichfeitige Fächer unterabge. theilt. Die Mündung hat fich an diefer Art noch nicht ausfindig machen lasen.

COLOR albus. FARBE weiss.

PATKIA mare mediterraneum ad $\mathrm{Li}$ burnum portum in Hetruria.

MENSURA ${ }_{3}^{2}$ lin, diam. $V A T E R L A N D$ das mittelländifelie Meer bey Livorno in Toscana. GRÖSSE 는 Linie in Durchme/fer.

\section{NAUTILUS ANGULATUS.}

\section{DER WINKELIGE SCHIFFER,}

T A B. 22. Fig. a. b.c.d.e.

IEsTa fpiralis involuta orbicularis utrinque elevata, fpiraliter firiata, friis multis parallelis a puncto eccentrico ema. nantibus valde arcuatis; anfroctibus quinque pedetentim crefcentibus (uti ex dimidiato fpecimine fig. $e$. apparet) dor $\sqrt{2}$ parte 21. n. 0 . ab angulo o areæ pun- fe' aus und find fehr ftark in die Runde ctatæ arcuatæ (ubi in aliis congeneribus gebogen; die fiinf Windungen nehmen ftuctura huic fubfimilibus orificium ar- allnsahlig an Gröse zu (wie aus einem

Die SCHALE if sinnirkelformig eings. rollt, kreisrund, auf beyden seiten er. haben, nach den Lauf der Windungen geftreift, die vielen Streifen laufen mit einander parallel aus einem auserhalb des Mitselpuncts befindlichen Punct frrah'wei$\mathrm{P}$ 
cuatum lineare apertum exftat) per dimidium peripheriæ ufque ad $m$. carinato, inde fefe in duas carinas pedetentim divergentes $m . p . q . \& m . r$. s. dividente, ficque triangulum æquicrurum acutangulum femifpirale conftituente, interjacente fpatio fuboblique levilime elevato, multis poris transverim feriatis pertufo; diffepimentis antrorfum convexis; thalamis multis (in hoc fpecimine dimidiato thalamis exterioris anfractus 27 , reliquorum quatuor anfractuum interiorum autem 77 , ergo omnibus quinque fimul fumtis 104 anguftis valde arcuatis, per ftrias extrinfece indicatis, iterum per innumeros ifflmulos fubæquidiftantes transverfim in totidem loculos minores fubdivifis, Orificium non diftinguibile, nifi ifti pori dorfales, uti faltem nobis videtur, forfan funt orificia fingula totiden fingulorum thalanorum.

COLOR albus.

PATRIA finus arabicus, in cujus te- sur Hälfte getheilten Stiick fig. e, er. hellet); der Rücken ift iiber die Hälfte des Unukreifes m. 11, o, vom Winkel o der punctirten gebogenen Fläche oder Feldes (wo an andern Gefihlechtsverwandten, die mit diefem einze ziemlich ähnliche Bauart haben, die bogenförnige frichformige offene Mïndung ift) bis all $\mathrm{m}$ gekielet, von $d a$ aus er fich in awcy all. mählig fich erweiternde Kiele m. p. q. und m. r. s, artheilet, und auf folche Art ein gleichfcbenkliches fpitwwinkeliges, halb. Schnirkelformig gebngenes Dreyeck bildet, der darzwifchen liegende Raum hat eine etruas fchiefe Richtung, if gar wenig erhaben, und durch viele kleine in viclfache Querreihen abgetheilte Löiher durchbohret; die Scheidewände find vorwärts gewülbt, die Kammern sahlreich (in diefem halbgetheilten Exemplar hat die äuserfte Windung 27 Kanmern, die übrigen vier innern Windungen aber 77, $1-$ fo alle fünf wufanmen 104 Kanmern), enge, ftark gebogen, die au swärts durch dic Sireifen angedeutet werden, und find abermahl durch uberaus viele kleine faft gleichweit entfernte Querwände in foviel klcine Fächer unterabgetheilt. Die Mündung ift nicht zu erkenuen, wofern nicht etwa jene kleine Löcher ïber dent Riicken, wie es uns wenigftens wahrfcheinlich ift, die einzelnen Mündungen eben fo vieler befonderèn Kammern find.

FARBE weiss.

$V A T E R L A N D$ der arabifche Meer. 
fris majoribus fpecimina a D. Spenglero bufen, in defen gröfsern Schneckerzfchalen inventa, diefe Beyfpiele vonk Hrn. Spengler gefun* den wurden.

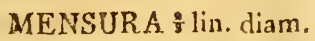

GRÖSSE $\frac{2}{3}$ Linie int Durchw.

\title{
NAUTILUS ADUNCUS.
}

\section{DER EINGEKRUMMTE SCHIFFER。}

\author{
T A B. 23. Fig. a. b.c. d.e.
}

a. magnitudo naturalis.

b. vitro auctus $\alpha$, a latere; $\beta$, a fronte; $\boldsymbol{\gamma}$. a tergo; \& dimidiatus. a. natürlicle Gröjse.

b. vergröfsert $\alpha$. von der Seite; $\beta$. van vorn; $\gamma$. vom Ricken; ১, zur Halfts angefcbliffen.

$\mathrm{T}$

ESTA fpiralis fubinvoluta, fubovata feu fubauriormis, ambitu ninirum ab angulo retufo feu leviter incifo $o$ per $p$. $q . r . s . t$. usque ad angulum obtufe prominentem \& leviter incurvatum $u$, id eft per fex octantes totius peripheria rotuudato, ab u usque $x$ per unum octantem retracto \& ab $x$ usque $o$ iterum rotundato, ntrisque lateribus non in medio fen centro, fed eccentrice inter centrum $\&$ ambitum mediocriter convexis, puncto

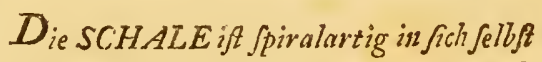
gewunden, zienlich eyförmig, oder zielmehr gewiffer Massen ohrformig, indem nehnlich der Unffang von de en eingedriick. ten oder feicht eingefisnittenen Winkel o iiber p. q. r. s. t. bis zu dem ftumpf hervorragenden und etwas ungelirimmten Winkel u, das ift $6 / 8$ des ganzen Un:kreiJes der Sshale betragend, in die Runde lauft, von "laus aber bis x ein Achtel lang eingezogen oder einwärts gebogen, $\mathrm{P}_{2}$ 
eccentrico unius lateris oblique oppofito puncto alterius lateris; de reliquo fere complanata (fig.e.), fpiraliter ftriata, firis permultis fubeleratis parallelis, a puncto eccentrico ad extima firiæ five ultimi articuli inflexionem (o) proximiore emanantibus valde arcuatis; ita ut fere tres quadrantes peripheriæ occupent; anfractibus tribus repente crefcentibus (fig. c.), quod apparet, fi cotis attritu aperiuntur; dor $\int 0$ per duas partes octantes o. $x_{0}$ u. obtufe carinato (fig. d.) per reliquos vero fex octantes $u . t . s . r . q \cdot p . o$. latiore lineari fubplano utrinque acute anigulato (fig. e.), ad o. (fig.d.) bifurcato, utraque parte attenuata arcuata introrfum vergente, plano intermedio fubtilibus punctis pertufo; diffepimentis antrorfum convexis; thalamis multis (hic 43) fecundum gyrationes longiflimis, fed transverfim valde auguftis friis externis refpondentibus maxime arcuatis, denuo per ifflmulos numerofos tranverfos in totidem loculos minutos (hic circiter mille, ecquis tempus oculosque fcrupulofiore numeratione perdere velit, cım jam thalami principales oculi inermis acumini fuffugiant?) fubdivifis. Crallitudo dorfi latioris ad anguftiorem feu carinatam partem eft ut $3 \frac{x}{2}$ ad 1 feu $\eta$ ad 2. Orificiume, licet omni adhibita diligentia, oculis noftris hucusque obductum manet, ni forte ifta foraminula, quæ in dorfo confpiciuntur, uti jam ad fpeciem præcedentem dictum, fingula fingulorum und von $\mathrm{x}$ bis $\mathrm{u}$ des letate Achtel wieder auswarts angerundet ifi. Die leyden Seiten find wicht in den Mittelpunet, fondern auferhalb demfelben wwifiren ihn und den Unfang mittelncißig gerwölbt, und wwar Jo, daß die cine eccentrifcine Wölbung der einen Seite jener der andern fohief entgegen liegt; das iibrige der Schale ift faft flach (fig. e.), durchaus aber hat fie viele neben einander gleichweit laufende, etwas erhabene Sireifen, die aus dem eccentrifshen genteinfonien Puncte der Woibung einer jeden Seite, der iler Einbeugung des letatenStreifens oder des letaten Gliedes (o) naiher ift, fpiralartig aus. gehen und fo fark gebogen find, dafs fie ungefaihr drey Viertheile der ganzen Peripherie einnehmen. Man findet beyn Auffohleifen drey an Weite folnell zunehmende Windungen (fig. c.); zwey Achtel dies Rückens o. x. u. find fun pf gekielt (fig. d.), die iibrigen Jechs Aihtel u. t.s.r.q. p. o. find ziemlich breit, frichformig, faft flach, auf beyden Seiten Scharf winkelig (fig. e), bey o. (fig. d.) theilet fich der fache Rïcken gabelformig in wwey Theile, und wendet fich auf jeder Seite nit einem fich vordiunernden Theile gebogen einwairts, diefe Fläche des Riichens ift mit vielen felr feinen gereiheten Puncten durchlöchert; die Scheidewände find vorwairts convex; die vielen Hauptkammern (hiev 43) find ftarh gebogen, inden fiefish fehr lang nach dem Lauf der Windungen herum biegen, find aber in die 
thalamorum orificia fint.

COLOR albus.

PATRIA finus arabicus, in cavitate teftarum majorum fpecimina a D, Spengler detecta.

MENSURA $1 \frac{x}{3}$ lin. long. $\& 1 \frac{2}{2}$ lin. lat.

Nota. Dantur etiam fpecimina convexiora, dorfo latiora \& bifurcatione expreffiore, hocque modo ad Nautilume angulatum magis approximantia, a quo vero præter ceteras notas præcipue numero anfractuum fatis diftinguuntur.
Quere fehr fchmal and machen fich von aufsen durch die erhabenew Streifen kenntlich, von welchen fie bedeckt werden. Diefe Hauptkammern find durch fehr zahlreiche kleinere $Z$ wifchenwände nach der Quere in eben fo viele kleine Fächer (hier ungefähr ïber taufend, wer wollte Zeit und Augen mit allsu genauem Zählen verderben, da Shon die Hauptkammern einem guten unberaffineten Auge entfiehent?) abernahl untergetheiit. Die breitore Dicie des Unfangs verhält fich zu der fchmälern oder kielformigen wie $3 \frac{2}{2} \approx u$ o oder 7 zu 2. Die Mündung bleibt aller angewandten Milise ungeachtet unfern Augen noch inmer verborgen, wenn nicht vielleicht, wie fchon bey den vorhergehenden Stïcke erwiihnt worden ift, jente durchlösherte Puncte des Rückens die einzelnen Mündungen fo vieler einzelnen Kammern find.

FARBE weijs.

$V A T E R L A N D$ der arabifche Meerbufen, vom Hru. Spengler in gröfsern Schneckenfchalen gefunden.

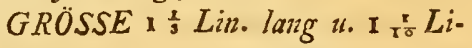
nie breit.

Anmerk. Man findet auch Beyjpiele von diefer Art, die gewölbtere Seiten, einen breitern Rücken und eine färkere gabelige Theilung haben, und fich auf folche Weife dem winkeligen Schiffer mehr nähern, von dem fie aber anfser andern Kennzeichen hauptfaichlich durch die Anzahl der Windungen hinlänglich verfchieden fónd. 


\title{
NAUTILUS MELO.
}

\section{DER MELONEN - SCHIFFER.}

\author{
T A B. 24. Fig. a. $-f$.
}

VARIETAS $\alpha$.

ERSTE ABANDERUNG.

Testa fpiralis involuta fubfphærica, lævis, polis parum elevatis; anfrattibus novem (in fpecimine picto, numerus enim aufractuum variat), extimo folum confpicuo; fuperficie longitudinaliter feu fecundum columellam vel ab uno axeos polo ad alterum per decem ambulacra feu coluros in totidem areas lanceolatas divifa (novimus \& polfidemus etiam fpecimina $7,8,9,11,12,13 \& 14$ areis) externe vix vifibiles, in interioribus anfractibus exprefliores 1 . diftinctiores, areis nimirum quafi articulos convexiusculos ambulacrisque diffepimenta fubcoarctata \& tamen leviter coftata repræfentantibus; qualibet area prædita laminis cavis feu tubulis depreffis linearibus transverfis contiguis, fingulis futura folum latcraliter a fe invicem fecretis. Hi tubuli quidem in areis cujuslibet fpeciminis numero æquales funt, fed non

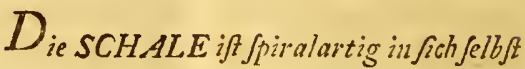
gesunden, beynahe kugelrund, nur an den beyden Polen etruas erhaben; Win. dungen find neun (in dem hier alggebilde. ten Exemplare; denn ihre Zahl ift veränderlich), davon bloß die äufserfite fachtbar ift; die Oberfliche theilet fich nach der Länge, das ift, längs der Spindel, oder von dem einen Pole der Axe bis zum andern durch zehn Gänge oder halbe Zirkel in eben fo viel lanzettförmige Felder (wir kennen und befitzen auch Stiiche von $7,8,9,11,12,13$ E 14 Feldern) die anfsen kanm sohtbar, an den Windundungen aber mchr ansgedriickt, oder deutlicher wu erkenmen find, da denn durch die Felder gleichfan etwas erhabene Glieder, und unter den Gängen verengerte, oder vertiefte, und doch fohwach geribbte Scheidewände vorgeftellet werden; auf einem jeden folchen Felde liegen in die 
in diverfis fpeciminibus; nam inter varia fpecimina noftra numerus tubulorum cujusvis areæ penultimi anfractus mox 24, uti in hoc fpecimine, $\operatorname{mox} 16$ in il10 , aut 20 in alio \&c. eft, ficque a duodecim usque ad 32 , in aliis forfan ultra afcendit, fed ab extimo anfractu introrfum per omnes anfractus usque ad tefte centrum feu ad medium axeos proportionate a 32 per 28,20 \&c. ufque ad 3 decrefcit. Utrum vero tubuli per omnes anfractus ufque ad centrum continui, vel ad minimum inter fe communicantes, an unius areæ tubuli ab alterius præcedentis vel fequentis areæ tubulis abfcifi \& fegregati fint \& fingulæ areæ feorlim exiftant, altioris indaginis adhuc res ef ; noftris faltem oculis, licet bene armatis, id detegere nondum licuit, prius nobis verofimillimum vifum eft. Orificiun nusquam apparet, nifi aperturce tubulorum in latere ultimæ areæ extimi anfractus forfan, \& ut nobis videtur, funt totiden orificia, quod hre teftulæ cum aliquot aliis Nautilis certa ratione habita commune habent. Id quod omne fimul fumtum totam earum ftructuram reddit prorfus fingularem, \& a reliquis omnibus teftaceis non folum, fed \& a dictis congeneribus valde diverfam, his tamen proxime accedentem. Interea cum ejusmodi corpuscula absque ullo dubio ad teftacea \& quidem ad fpiraliter involuta pertineant \& præ ceteris cum Nautilis noftris, inter hos auten proxime cum
Quere gleichbreite fchnale, an einander ftofsende hohle Blättchen oder flach gedrückte Röhrchen, wovon jedes blofs durch eine Nath an den Seiten von den andern abgefondert ift. Diefe Röhrchen find zwar in den Feldern eines jeden BcySpiels won gleicher Ansahl, aber nicht int verfchiedenen Beypielen ; denn unter den verfchiedenen Stïcken, die wir haben, ift die Ansahl diefer Rühchen eines jeden Feldes der vorletzten Windung bald 24 , wie in vorliegenden Exemplar, bald 16 an cinem andern, oder 20 wieder an einem andern, u. f. w. und fo fteigt felbige von 12 bis auf 32 , in andern vielleicht noch weiter hinauf; hingegen von der äufserften Windung einwärts durch alle Win. dungen, bis sun Mittelprinct der Schale oder der Mitte der Axe fallt fie verhältnifsnacsig von 32 , durch 28,20 , etc. bis auf 3 herab. Ob aler diefe Rührchen durch alle Windungen hindurch bis zun Mittelpunct unabgefet: in einem fortgehen oder wenigftens durch irgend eine Offnung unter einander Genneinfchaft haben, oder ob die Róhrchen eines andern entweder vorher gehenden oder folgenden $\mathrm{Fel}$ des abgefohnitten und abgefondert feyn, und folshemnach jedes Feld für fich allein beftehe, ift eine Sache, die einer weitern Unterfuchung ausgefatat bleibt; wir haben es wenigftens, auch nit guten Vergröfserungsglifern, wosh wicht entrieckeil können; das erfiere fcheint uns das Walvfcheinlichfte au feyn. Von einer Mündung 
duplicato-concameratis, in plurimis cha- if nivgends etwas au benterken, wofern racteribus conveniant, interim illa pone nicht etwa, und wie es uns foheinet, die hos appendicis loco pofuimus. Ceterum fi quis forte proprium ex iis conftituendi genus fit animo, lubenter in meliorem ejus fortiora noftris argumenta afferentis difcefluri fumus fententiam. Öfnungen der Röhrchen an der Seite des letzten Feldes der äufserften Windung eben fo viel Mïndungen find, welche $B e$ Schaffenheit etliche andere Nautilusarten mit diefer Schneckenart in gewiffen Verhältniffe gentein haben. Diefes alles zufammen vereinbaret macht, dafs man!den Bau diefer letztern für ganz befonders und nicht nur von allen iibrigen Schalthieren, foudern auch ihren vorgenannten Gefchlechtsverwandten fehr verfchieden, doch diefen ain erften ähnlich anjehen muss. Da indeflen diefe Körperchen ohne allen Zweifel au den Schalthieren, uad zwar au den in fich felbft gevundenen gehören, und vor andern mit den Nautilusarten, unter diefen aber an naichften nit den doppelt gekammerten in den nueiften Kennzeichen iiberein kommen, fo haben wir diefelben unterdefen diefen letatern als einen Anhang beygefellet. Sollte übrigens jemand etwa des Sinnes Seyn, ein eigenes Gefchlecht daraus zu machen, fo werden wir feiner Meynung, wenn er au deren Unterftütaung ftärhere Grïnde, als die unferigen find, bejbringen wird, nit Vergnügen beypfichten.

COLOR mox ochraceus, ab ochra - FAREE bald ochergelv von beygenifchferrea iisdem mixta, mox albus.

PATRIA: Adhucdum folfilia folum inventa funt fpecimina tem Eifenochèr, bald weifslich, in einigen ganz weifs.

$V A T E R L A N D$ : Bisher hat man fie bloss folfl gefunden, und zsuar

a) colore albo ad Brunn am Steinfeld

a) ganz weifs au Brumn an Steinfe.d 
Aufriæe inferioris ad confinia Hungariæ, ubi ex fonte in medio lacu cum aqua fcatente \& fragmentis aliorum petrefactorum rarifime provenit.

b), colore albo in lapicidina ad Grusbach (Kroisbach) prope lacum Neofidelienfem Hungaria in lapide calcario innumeris petrefactis, præfertim nautilitis minimis \& pectinitis, farcto.

c) colore albo, in lapidibus, ex quibus facellum ad S. Magdalenam Viennæ Aufria abhinc quadringentis amis redificatum erat, quodque Ao. 1784 in: cendio deftructum eft, ex quadam lapicidina fine dubio non procul a dita urbe difita, nunc oblita \& ignota.

d) colore intenfe \& dilute ochraceo, rarius albefcentc, in lapide calcario cinereo, granulis quarzeis mintitis mixto conchylioferô ex Tranfylvania.

e) colore dilute ochraceo, quandoque albefcente, ît arena partim folutà, pàtim leviter cohærente, prope pagum Unter-Pefchtifch (Al-Peftis') in Comitatu Hunyadenfi Tranfylvaniz. Vid. Joh. Ehr. von Fichtel Nachricht von den Verfteinerungen Siebenbürgens $\delta c$. Nürnberg 1780. P. I. p. 7 8. XXIII. 1).

Nota. Auctor defunctus, dum opus hocce fcribebat, pro Echinitarum . Ppe- in Unteröfterreich unweit der ungarifchen Gränze, wo fie fehr Jeltein aus einer mitten in eisenz Teich befuldichen Quelle mit dem Quellwafler und vielen kleinen Bruchftïclen von Milleporiten und fiznen Echinitenfiacheln hervor kommen.

b) weifs jn dem grofsen Kalkfeinbruch zu Grusbach (Kroisbach) an Neufiedlerfee in Ungarn, in einzem Kalkftein, der mit Millionen fehr kleiner Nontiliten und wit. telnuajsigen: Pectiniten roll gefillt ift, oder vielmehr gans daraus befteht.

c) weifslich aus den Kaikfteinen, woraus die im Fahre 1784 abgebrannte Magdalena-Kapelle auf den Stephansplatze $\approx u$ Wien in Öeferreich bereits : or 400 Fahren erbanet war, aus einem vermuthlich nicht weit davon befindlich gewefenen, nun aber gans vergeffenu Steinbruch.

d) theils blafs, theils hoch ochergell, jeltner weifslich, in einem afchgrauen mit kleinen Quarakörnern gemifusthten, noch verfohiedene andere Schalthiergehänfe enthaltenden Kalkftein, aus Siebenbiirgen.

e) blass ochergelb, zuweilen weifslich, in einem theils lofen, theils mit Kralk etwas aufammen hängender Sand, $\frac{1}{2}$ Stun-de vou Unter-Pefchtifch (Al-Peftis) in der Hunyader Gejpannfshaft in Siebenbuirgen. Siehe Foh. Ehr. von Fichtel Nachricht ron den Verfeinerungen Siebenbürgens etc. Niinnlerg 1780, 4. I. Th. S.78. XXIII. .

Anmerk. Der fe?. Verfafer hielt $d c^{-}$ mahls, da er diefes Werk forrieb, die- 
cie hæc corpufcula habuit \& Kleinii Echi- Je kleinen Körper fiir eine Alt ron Eih:nodermatum tabulæ XIma figurim D niten und. $\approx 0 g$ ans Kleins Meerigel-Behuc allegavit. Aft iconis hujus accurata fchreibung die Figur D dir. YI. Tafel comparatione cum defcriptione figuris- hiersu an. Allein ley genauler $Z u f$ crnt: que noftris facile invenietur differentia. menlaltung diefer Abbildung mit unferer Ab ifto tempore etiam mentionatus auc. tor fententiam mutavit, licet non publice declaraverit.

f) colore albo in lapide fuillo (vulgo marmore) nigro ad Duinum in littorali Auftriaco maris adriatici.

MENSURA $\frac{r}{5}-\frac{4}{3}$ lin. diam, maj. f. longitud, axeos. Befchreibung und Figuren wird man den Unterfaried leicht finden. Seitdem hat auch gedachter Antor feine Muysurig geäudert. nur nicht öffentich bekannt genucht.

f) queifs in firwarracm Stinkftein (iu?go Marmor) von der Kiifle des adriati. fchen Meeres bey Duino in öfterreichifchen Kiiftenlande.

GRÖSSE $\frac{2}{3}-\frac{3}{3}$ Linie in grösern Durchmeffer, oder nach der Länge der Axe.

\section{Explicatio Figurarum.}

Fig. a. magnitudo naturalis.

l. integra anfractu extimo adlunc s, vitro multum aucta.

c. s acies penultimi fen proxime interioris anfractus, ultimo $\int$. extimo anfractu demto, longitudinaliter $f$. fecundum axin repræentata.

d. Talis, fed ab apice fo $_{\text {ab }}$ uno axeos polo infpecta.

e. Facies tefta transverfim per centrum f. medium axeos angulo recto per futusam inter duos tubulos dimidiate.

f. Tefta longitudinaliter per centrum dimidiata, ubi tubuli omnes difiecti apparent.

Erklärung der Figuren.

Fig. a. natirliche Gröfse.

b. Die ganze Schale, die noch ilire äufscrfte Windung hat, ftarh revgrüfsert.

c. Aisicht der vorletzten oder näch/t innern Windung, nach slbiehmung dor letsten oder ciufserften, nach der Länge oder wach der Axe zorgeftellet.

d. Dergleichen, aber vom Wirbel, oder von dem Pole der Axe anzufehen.

e. Die Schale in die Quere durch den Mittelpunst, oder rechtwinkelig uber die Mitte der Axe, durch eine wwifchen zwey Rohrchen durchgehende Nath zur Hailfte getheilet.

f. Diefelbe nach der Länge durch den Mittelpunct getheilet, wo alle Röhrohen durchfinnitten ergheinen. 
T A B. 24. Fig. $g$.

IHec varietas a præcedenti non differt, Diefe weicht won vorhergehender blofs nifi forma ad ambos polos magis depref- darin ab, dafs ihre Pole mehr miedergefa \& tubulis transverfis proportionate driicht und die Querröhrchen verhailtpancioribus, licet magnitudine teftæe nifsmä̈sig weniger find, obgleich die Grö. xquali.

se der Schale von beyden gleich ift.

COLOR \& PATRIA uti lit, e) Var, $x$.

FARBE und VATERL AND wie bey Lit. e) der erften Varietät.

MENSURA $\frac{5}{2}-$ lin. diam. GRÖSSE - Lin. in Durchm. 


\section{ERRATA ET EMENDATIONES.}

jag. lin.

$$
\text { pro }
$$

Vji 3 Præf. fic etinn Bafcbius

18 ult. larus ".

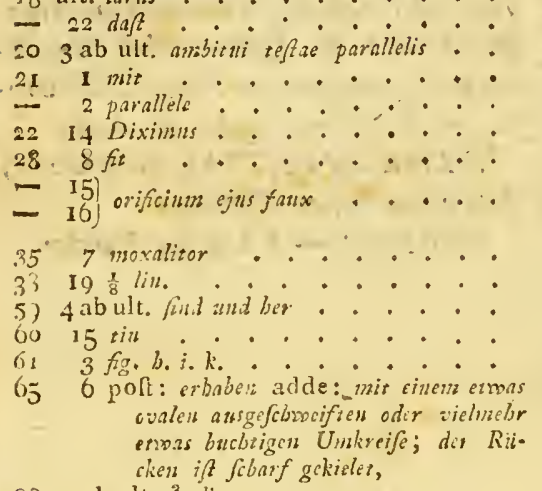

885 ab ult. ${ }_{4}^{3}$ diaml. . . . . . .

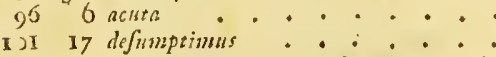

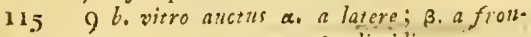
tc; $\gamma$. a zergo $; \delta$ dinzidiatus.

\section{etiain Batfcbius}

maxchmabl unterbrochen if, oft in

latus

dafs

ad anbituns teftac perpendicularibus et obliquis, $\approx n$

Senkrecbte and foliefe,

Diximuts

fit

orificium Spectatori ad dextram apparet ita, ut cjus faux

\section{mox alizer}

$\frac{1}{3}$ lint.

find bin and ber

titi

fig. a. b. c.

\section{lege}

et germanice:

$\frac{3}{4}$ lin. diam.

alleta

definufinnus

b. vitro auctus a latere; $d$. a frouse; e. a sergo; c. dinidiatus.

b. vergiöfsert a von der Seite;

$\beta$. von vorn; $\gamma$. vont Kicken;

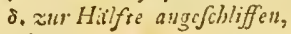

$116 \quad 1$ von $x$ bis $u$

- 12 (f.g.

Q1 (fig. do)

Is 8 abult. Windungngen . . .

12118 dit

122 b. vergrefsert von der Seite; d. von vorn;

e. von Rücken; c, zur Hälfic angefcbliffen;

von $x$ bis 0

$(f$. $.6, d$.

(fig. b. d.)

inner'n Windungen

dicta

figurain 
Tot..

(J)

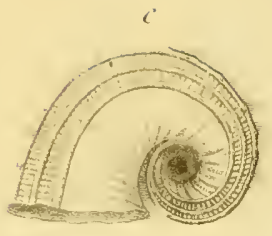

c

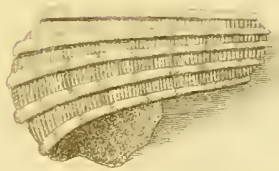




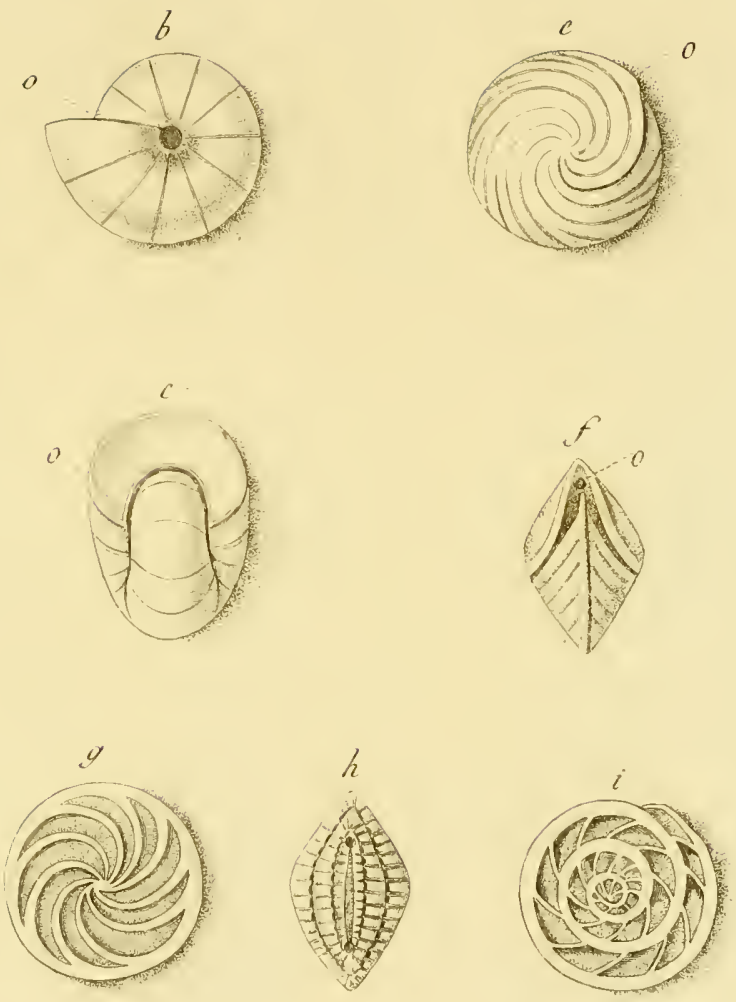



$$
\begin{aligned}
& 9 \\
& 8 \\
& 8
\end{aligned}
$$



.74 . 4.
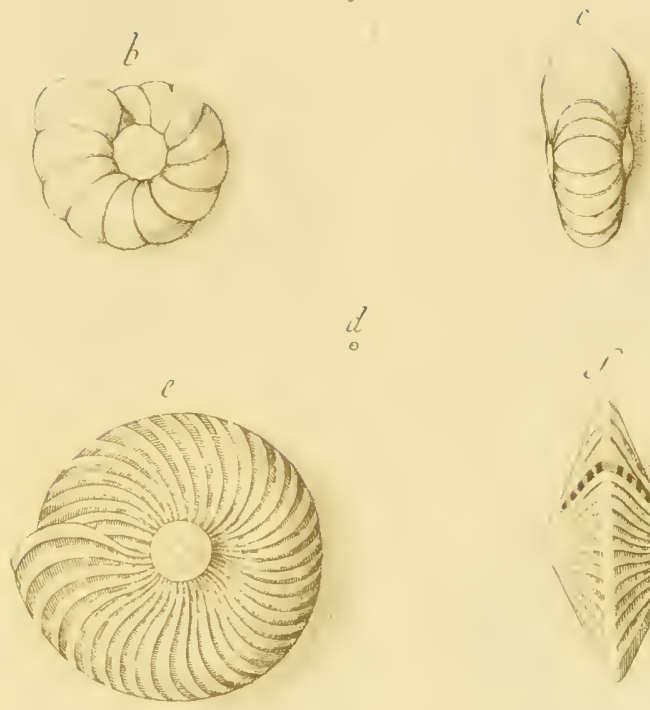

it
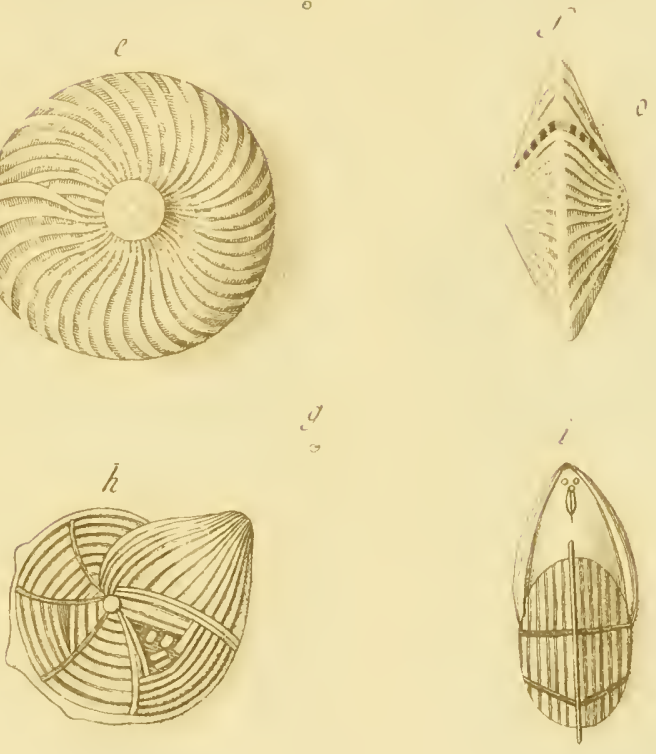


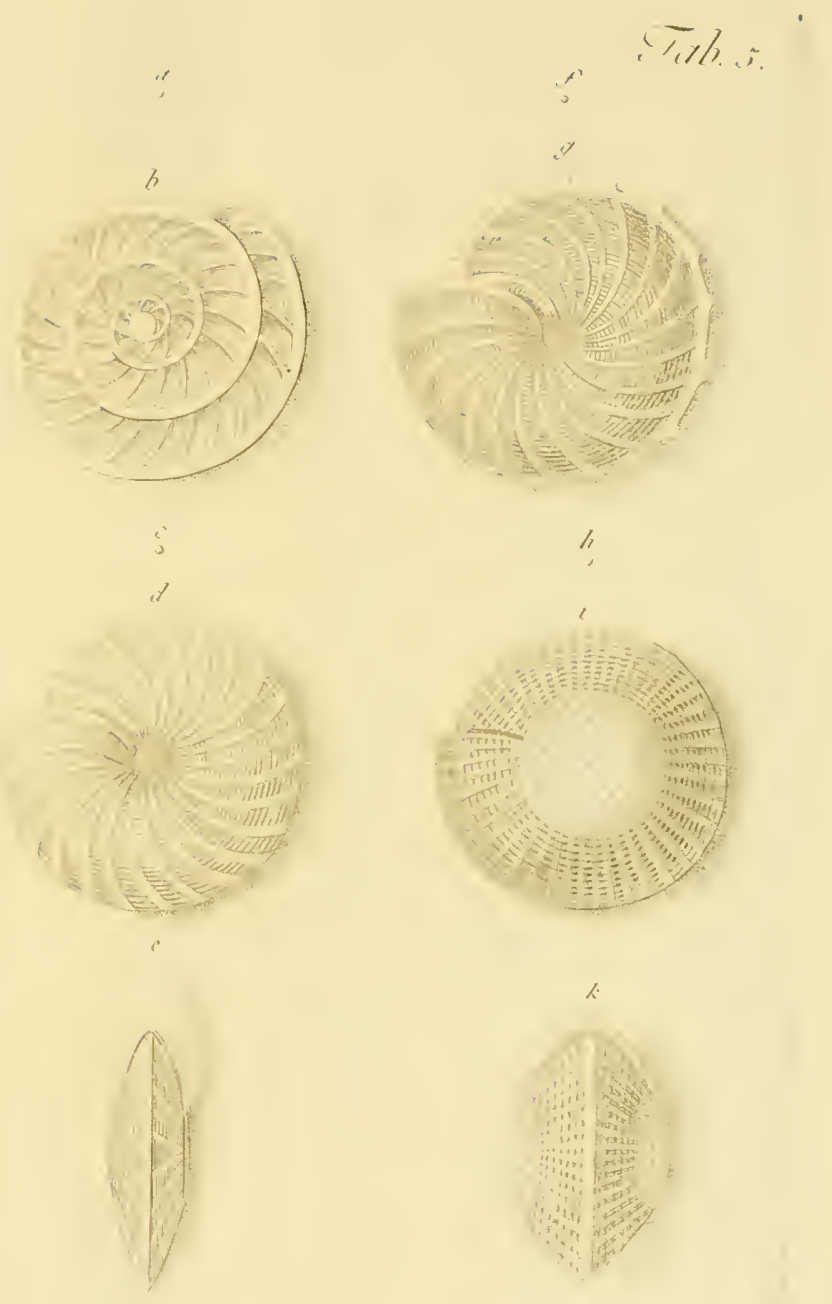

¿

e

Tab.

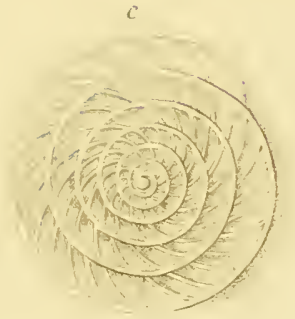

$d$

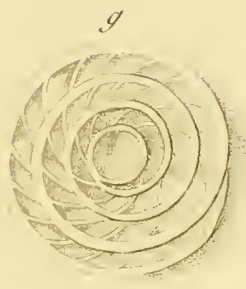

h 

c

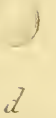

d
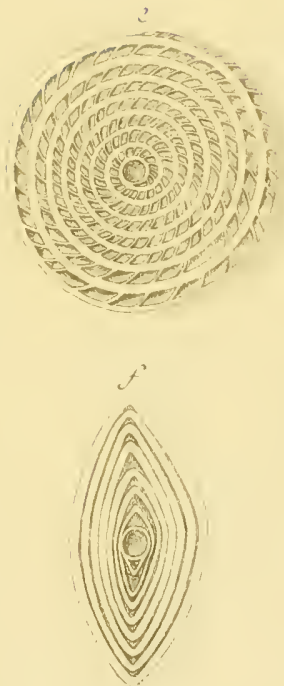

Tith. 7.

$a$

b
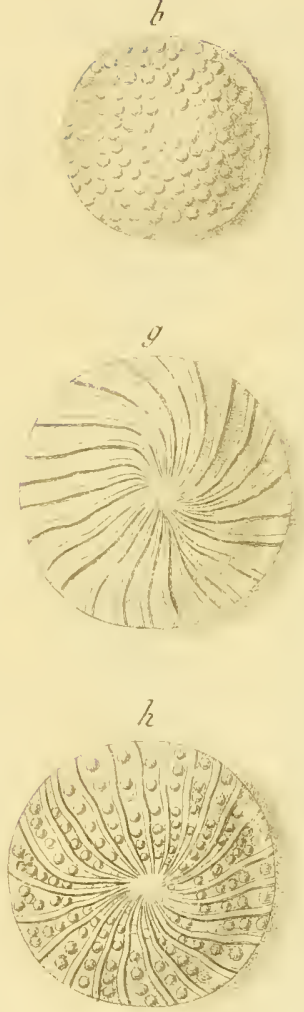



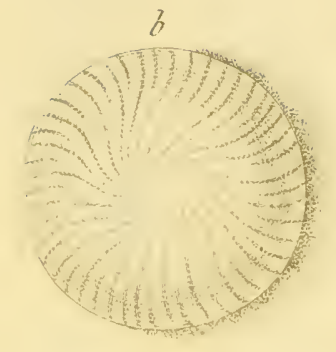

Taho.
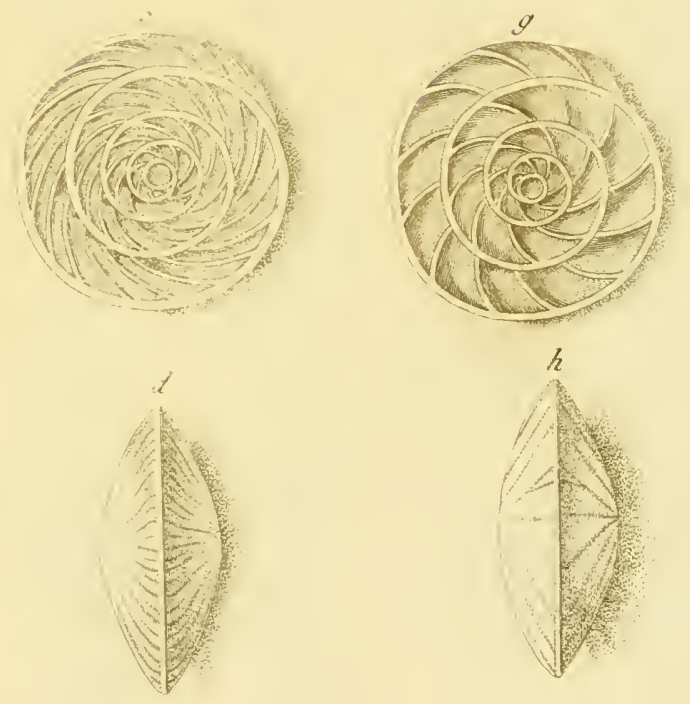

sitho i
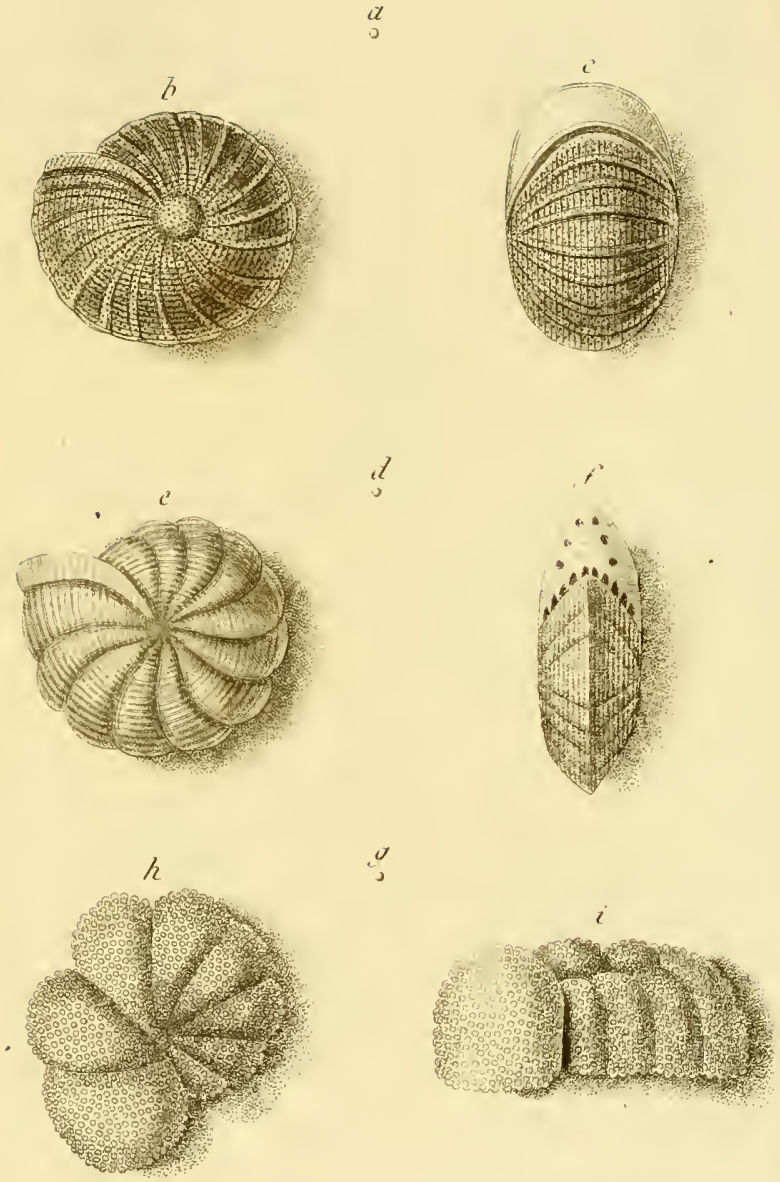



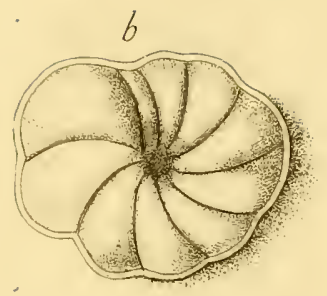

$a$

Tab. 10.
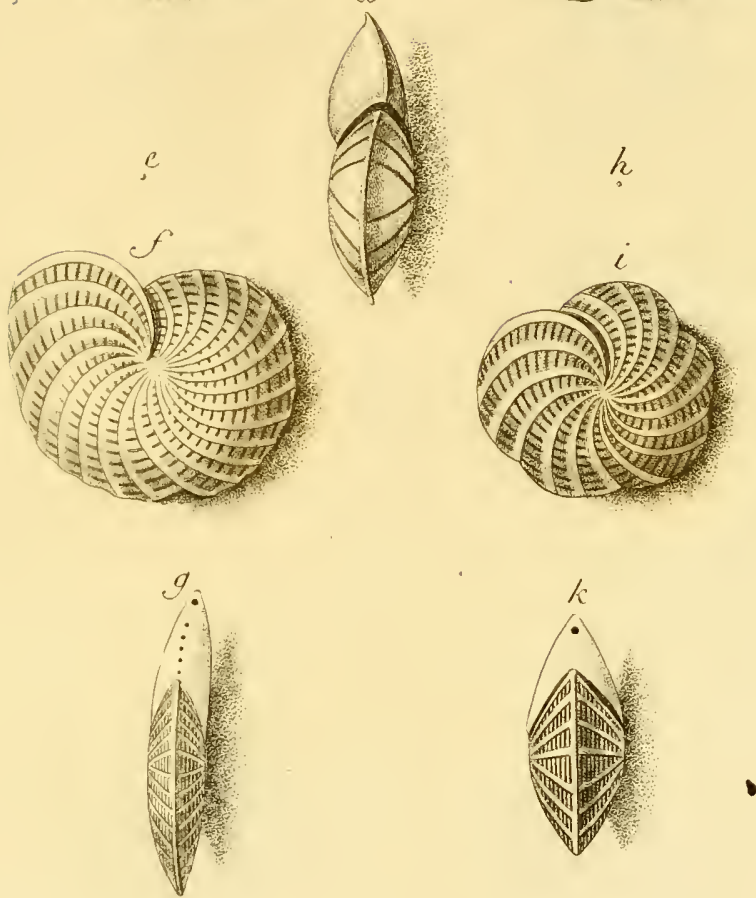

d)

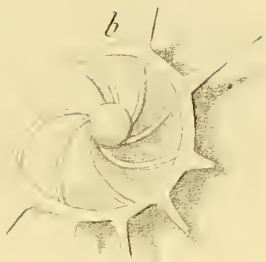

$c^{*}$

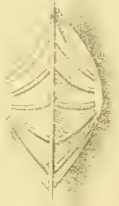

$y$

सैद्ध

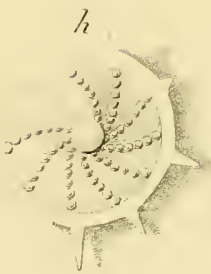

$d$ iab.11.

ve

c

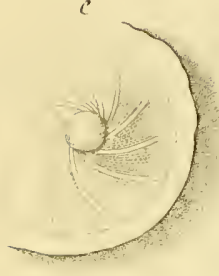

$t^{2}$
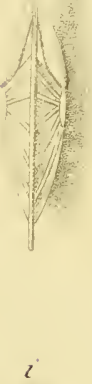

is

$k$

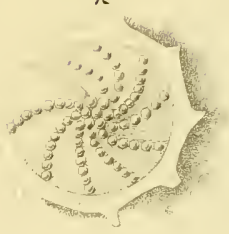





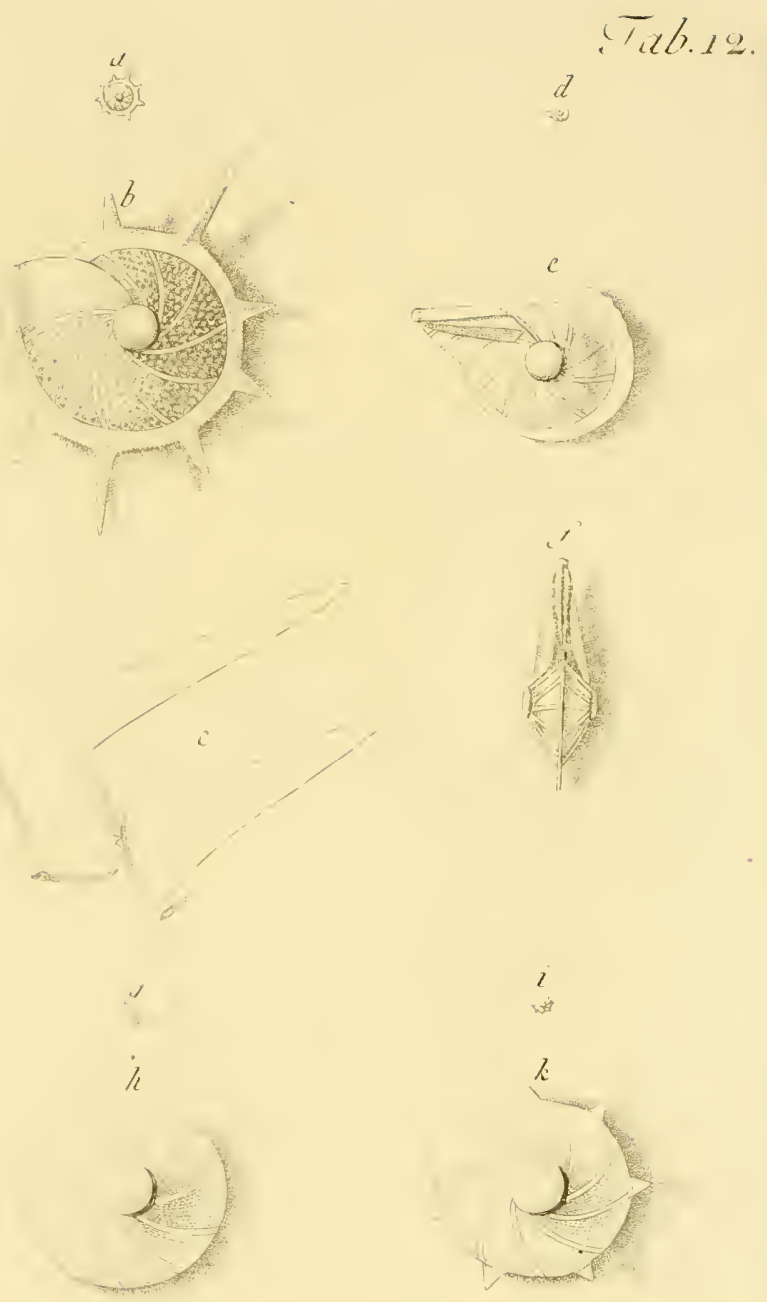



(j)

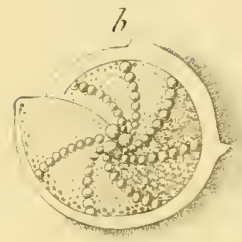

i

(1)

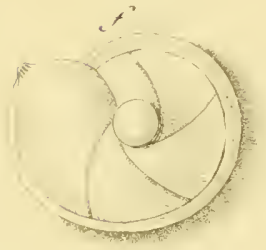

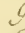

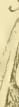

(6)

鲜

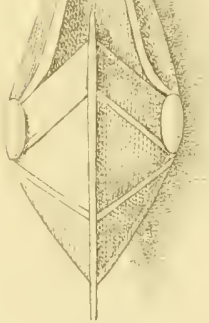

i

Tab.s.

tis

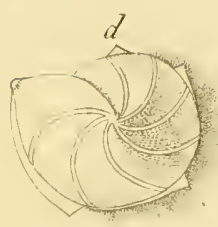

le

앙

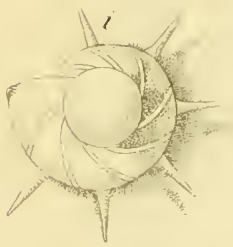

k

(5)

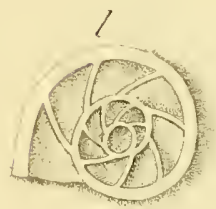



Tab.14.

$a$
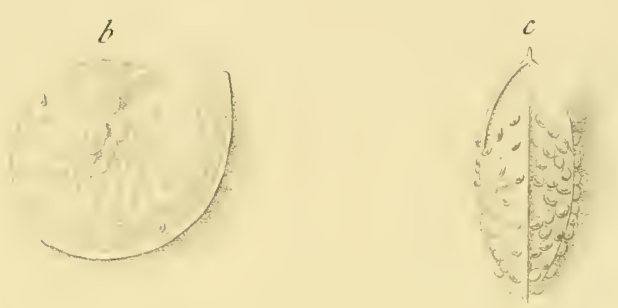

Tab. 15
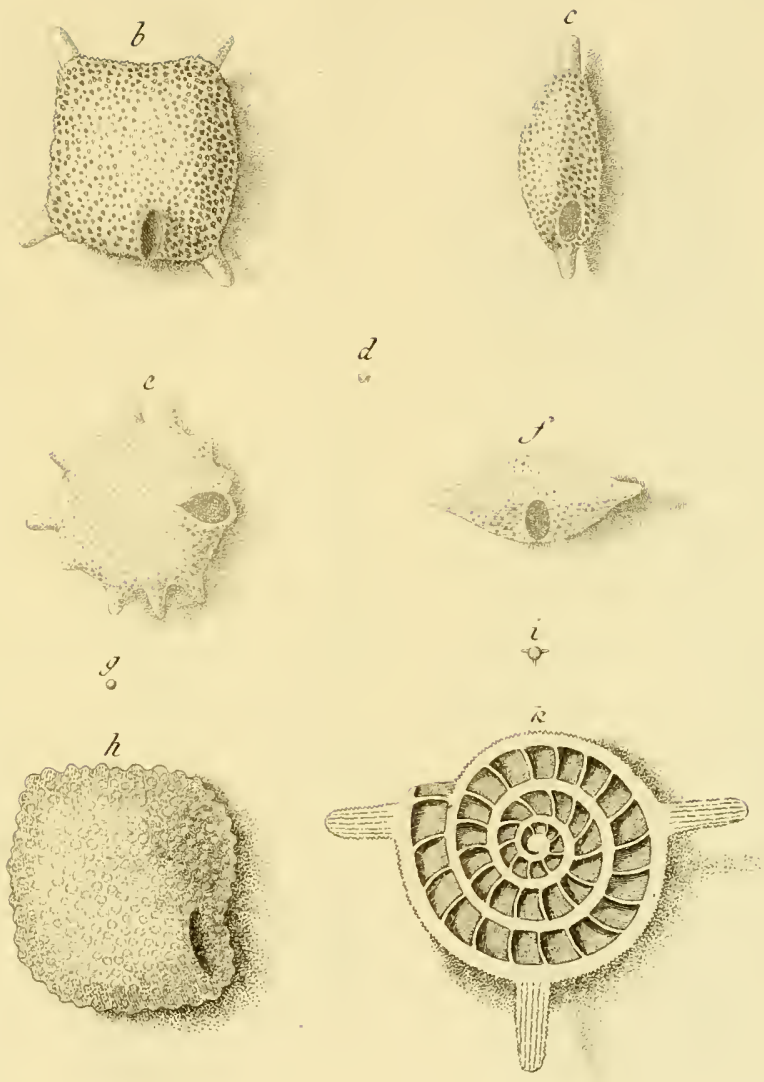

$\frac{a}{1}$ 



\section{a}

b
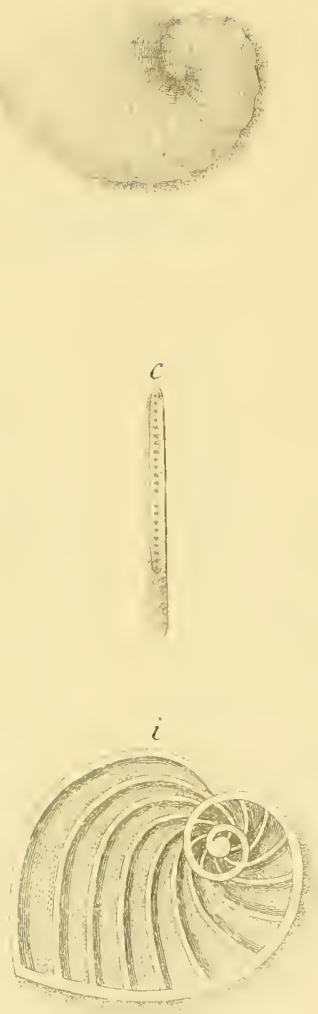

Tablot.

d

e

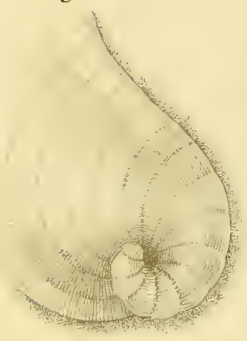

f

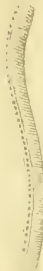

4

h 



\section{a}
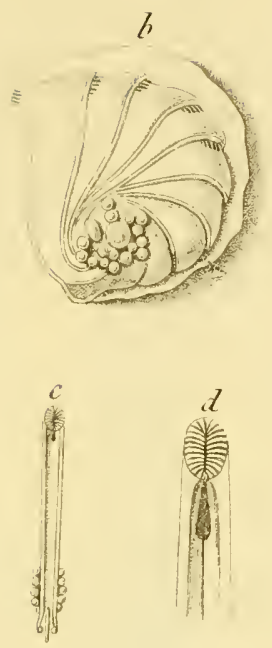

h

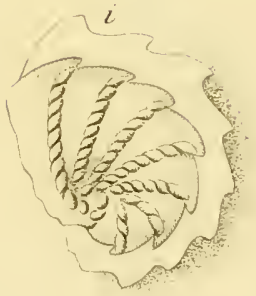

$$
\text { crab.17. }
$$

(i)
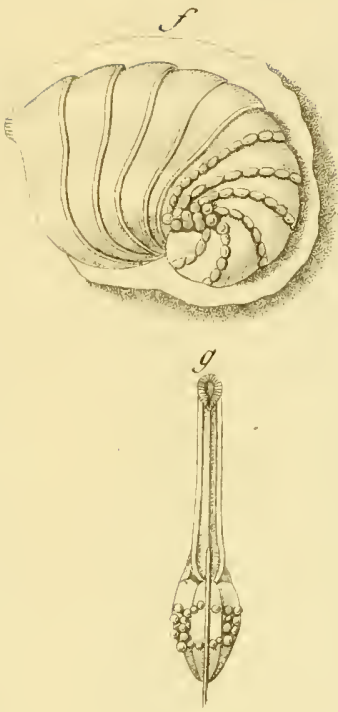

싱

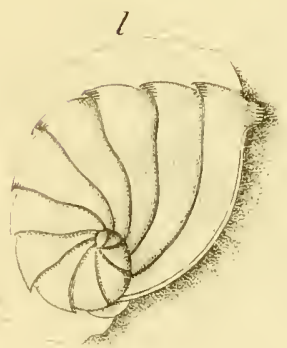



$a$

b
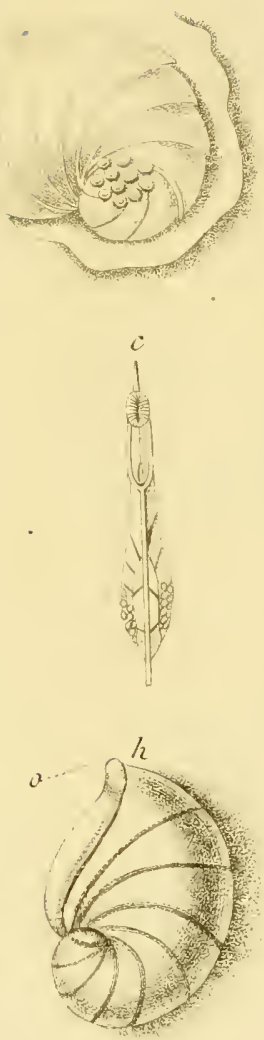

Tab.12.

$\frac{d}{\infty}$

c
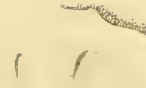

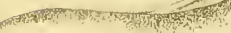
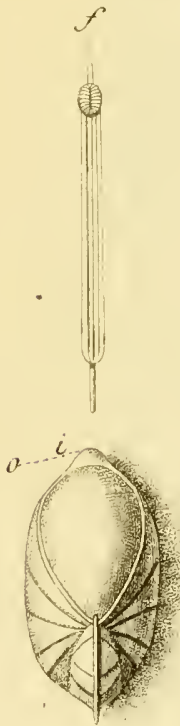


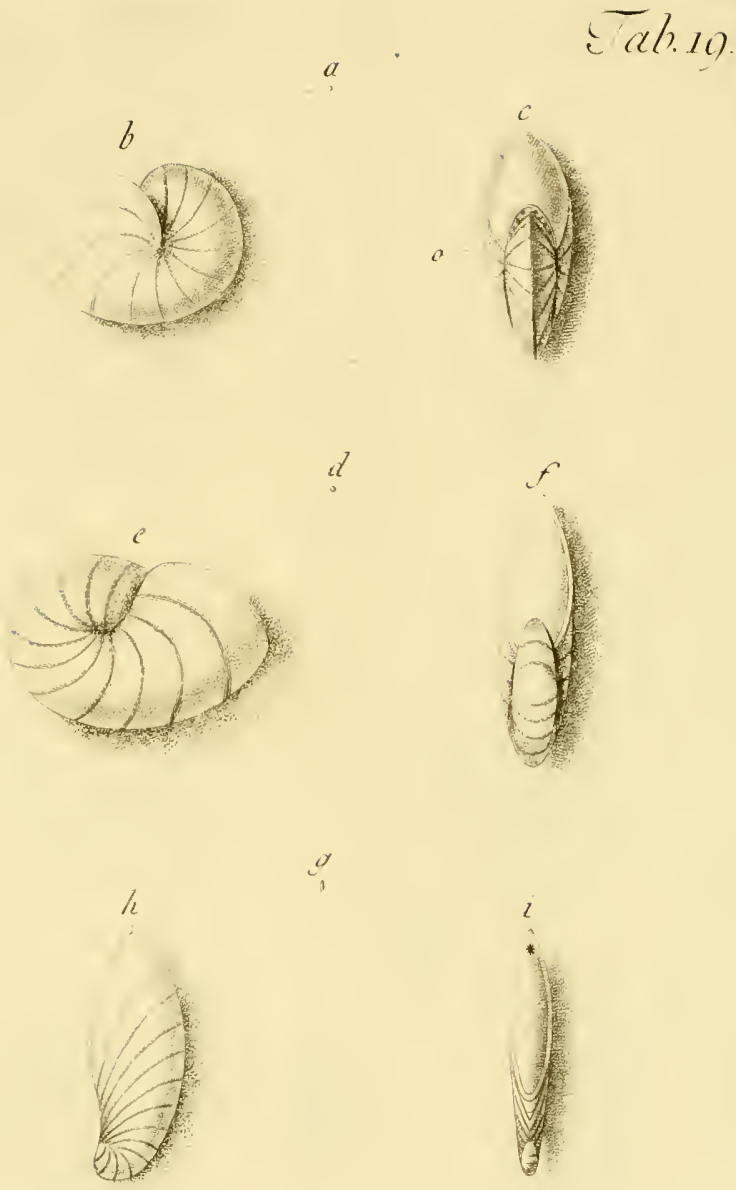



\section{Tabo?}

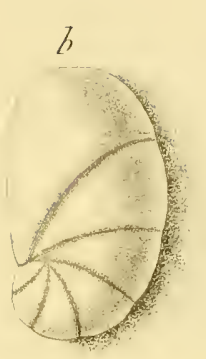

at
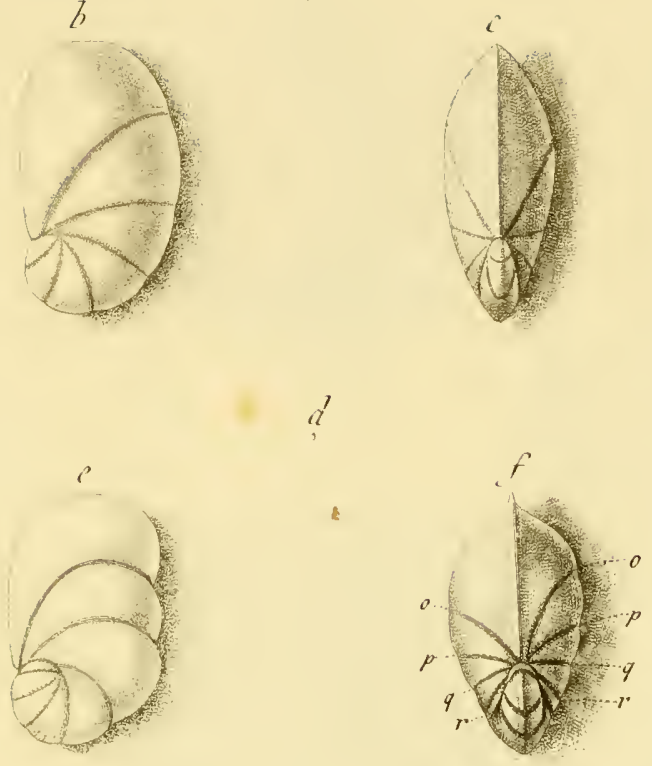

$$
\text { व }
$$
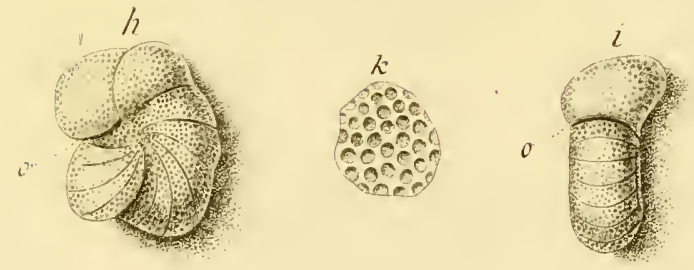



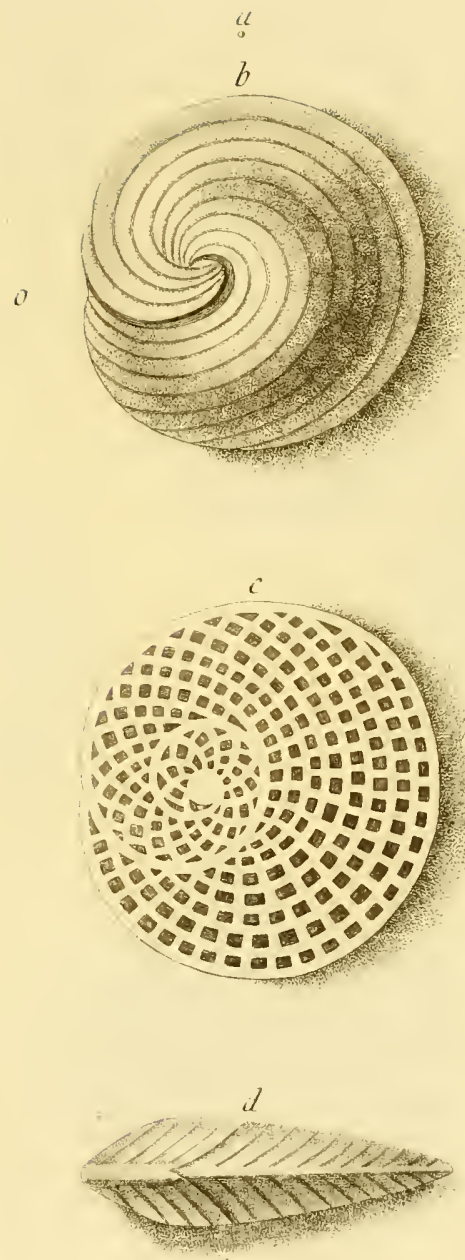

Tallog. 



\section{Tab.22.}

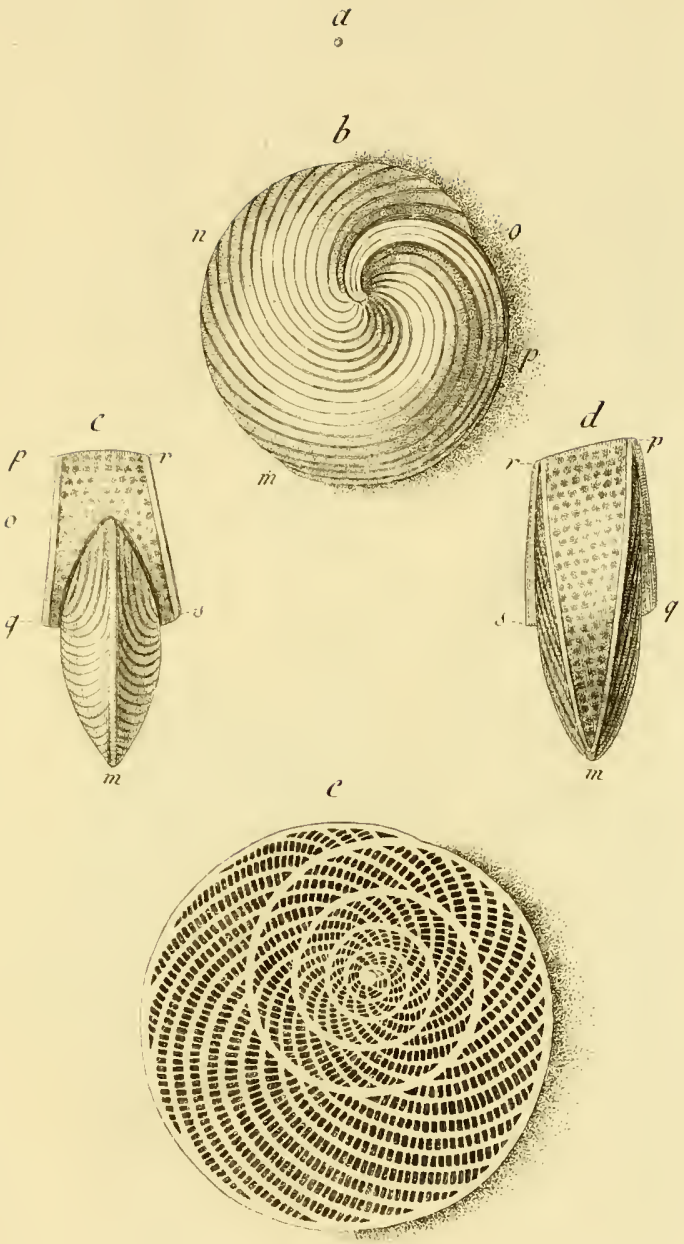




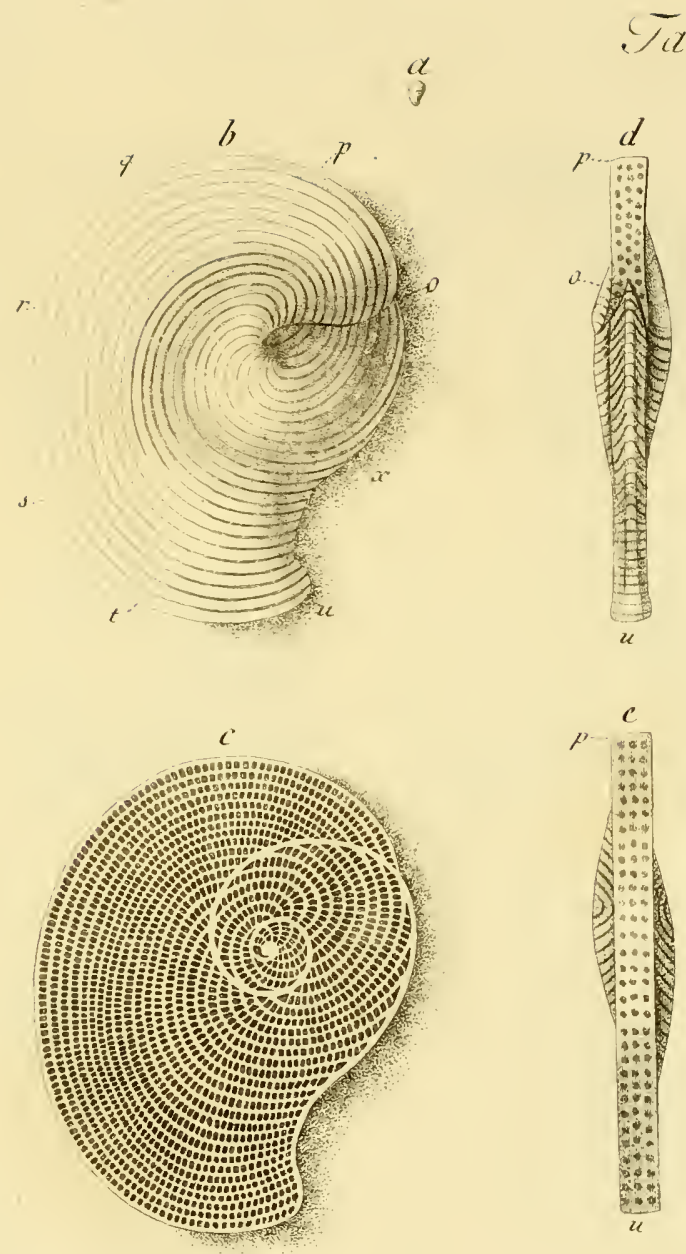



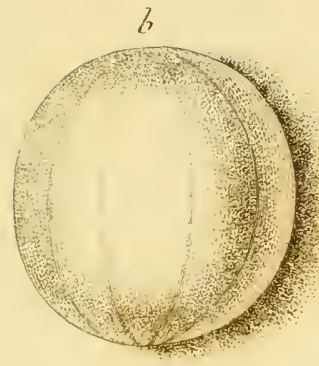

$0_{0}^{2}$

(at) 24.
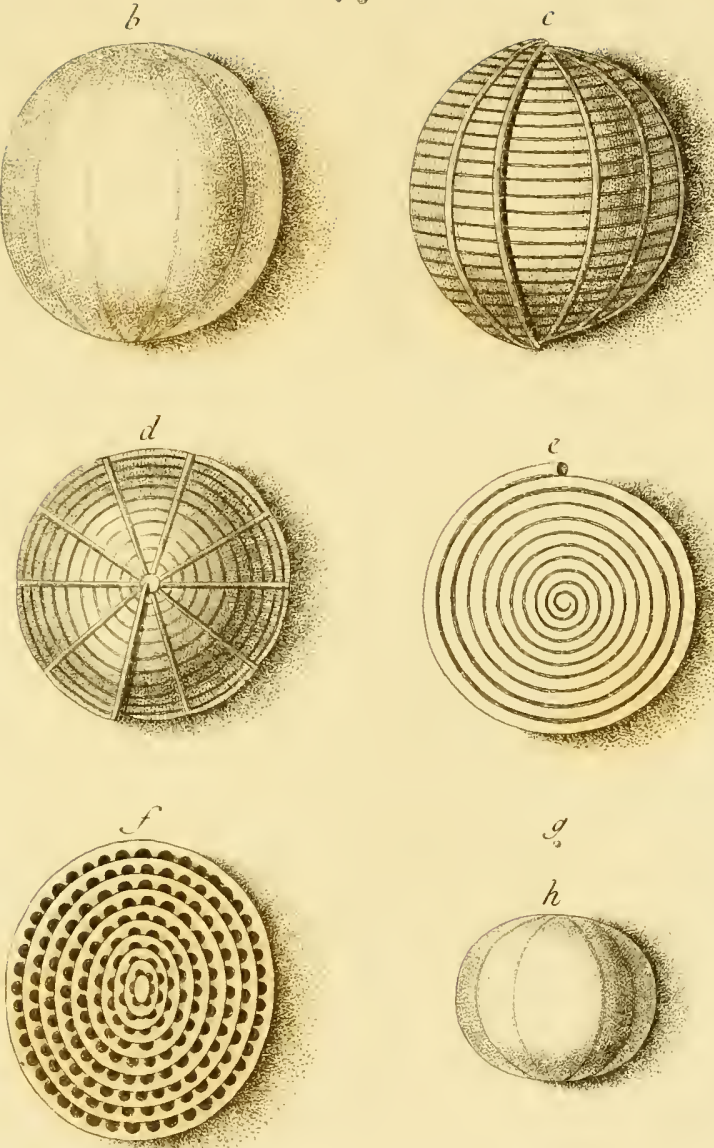




$x_{7}$ 2.

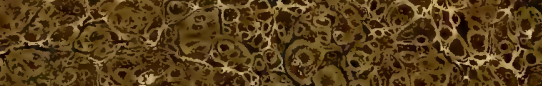

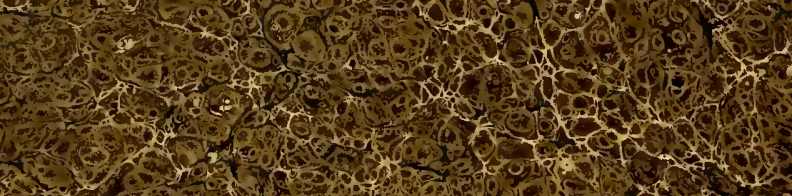

. 2.6. 2.3.

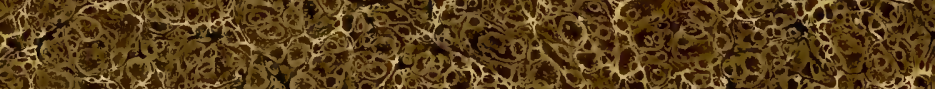

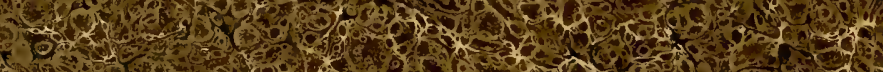

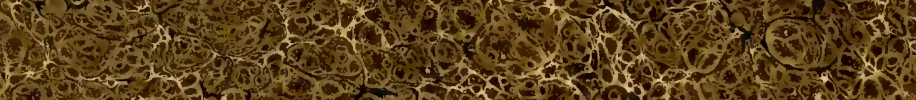

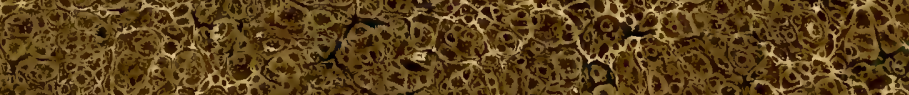

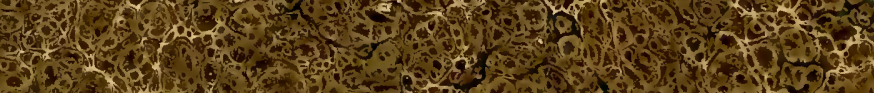

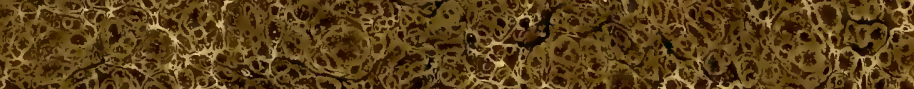

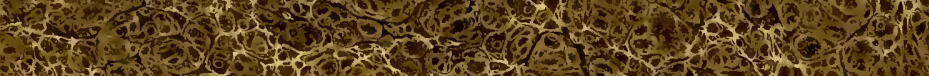
r. H.

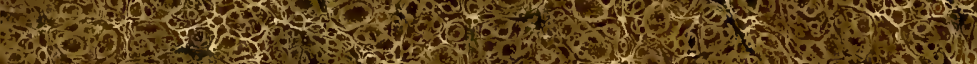

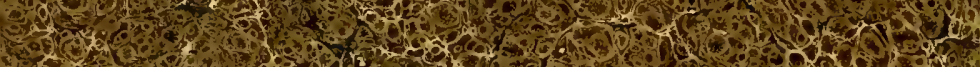

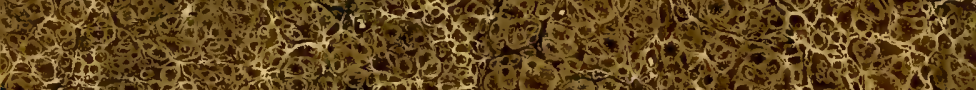

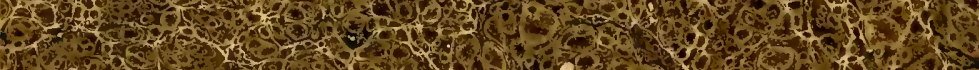

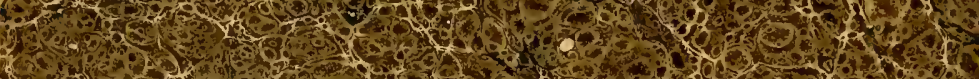

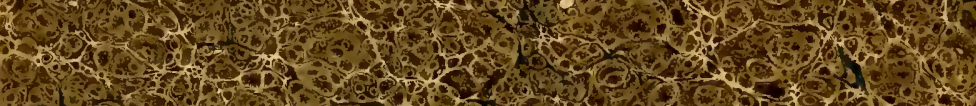

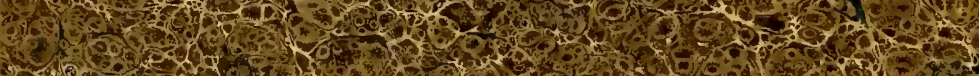

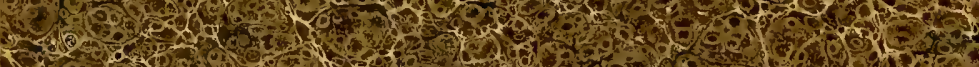
Wh

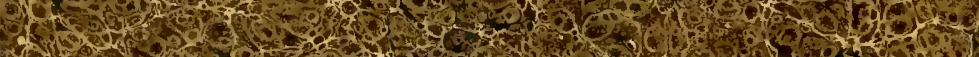
2.1. S.

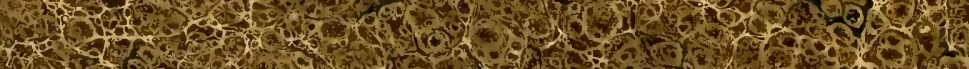

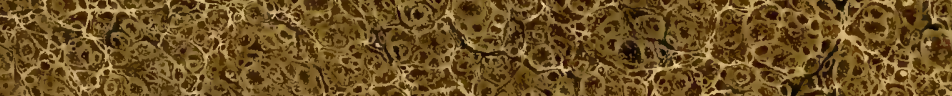

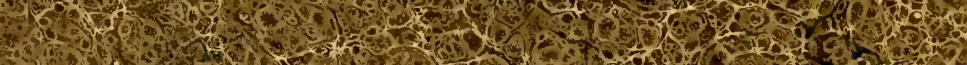

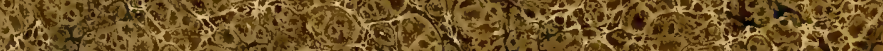





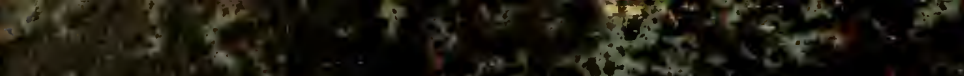

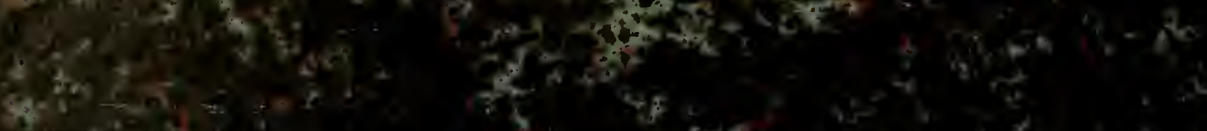

Qt.

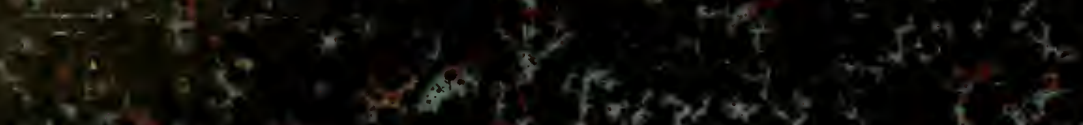

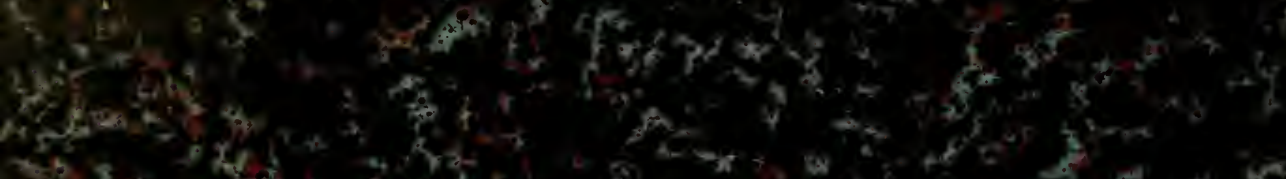
(3) $3 x^{2}$

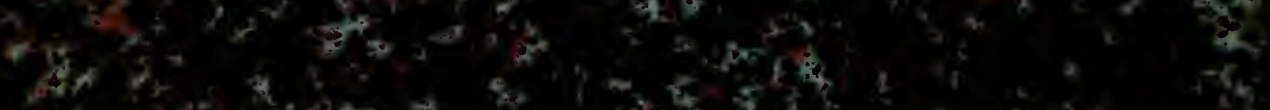

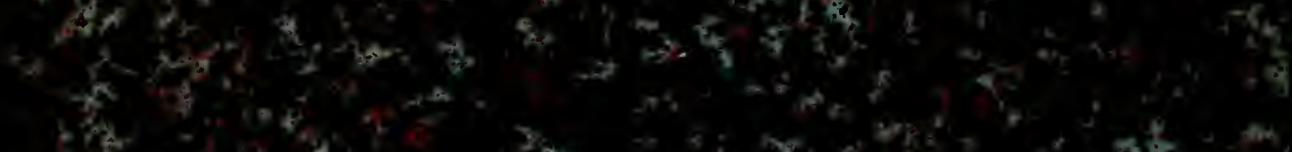

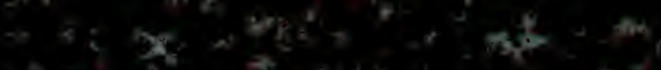

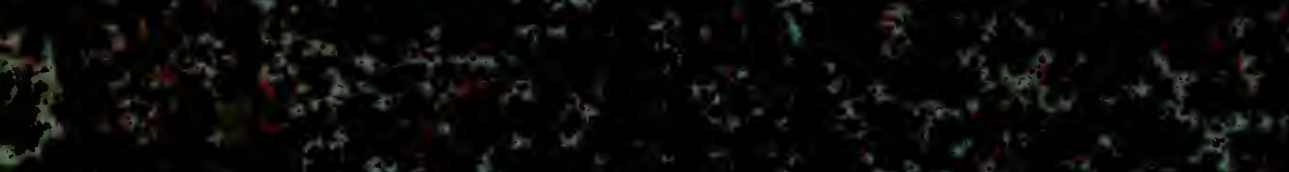

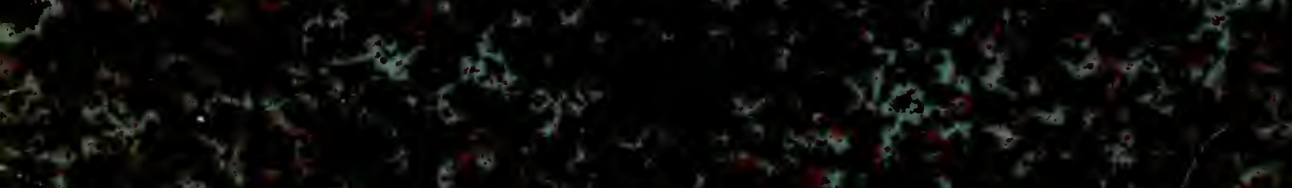

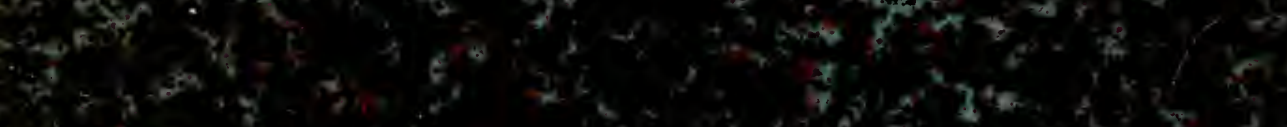

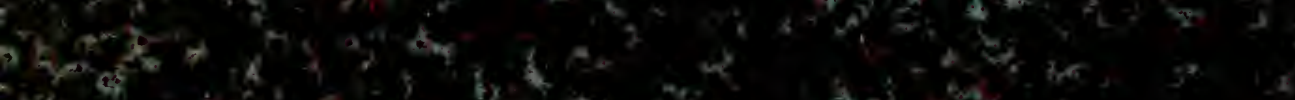

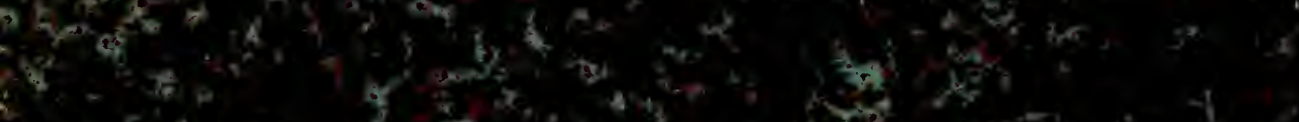

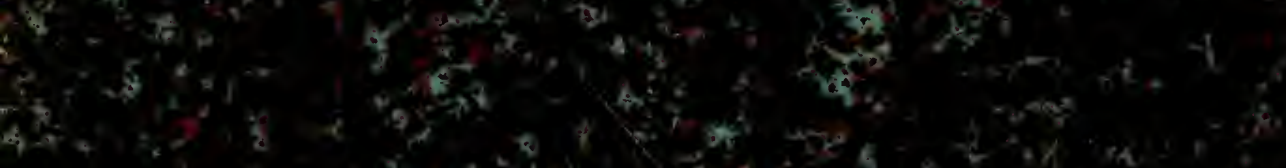

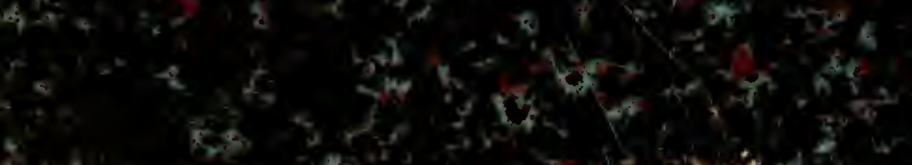

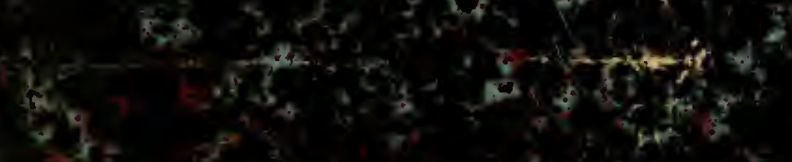
and C.

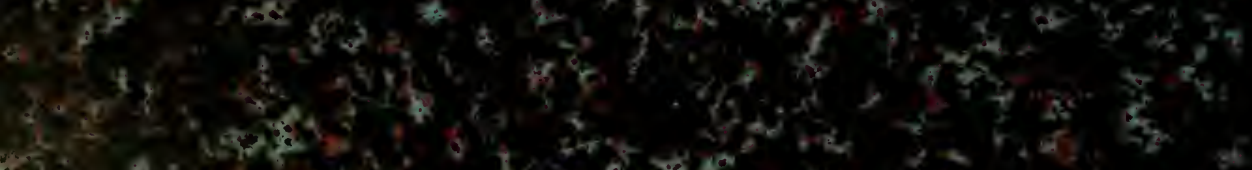

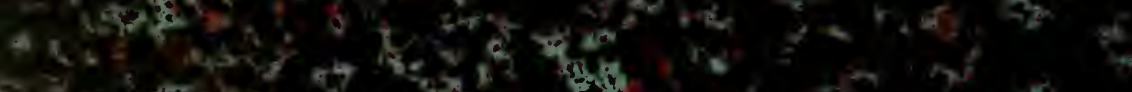

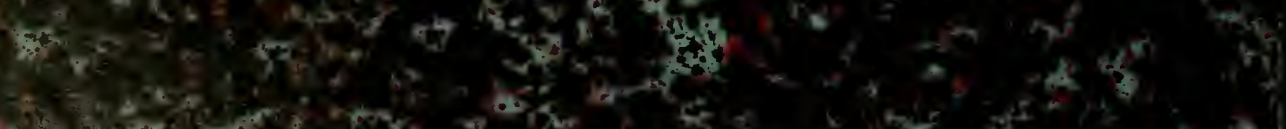
W 